ESAIM: COCV 19 (2013) 844-887

DOI: $10.1051 / \mathrm{cocv} / 2012036$
ESAIM: Control, Optimisation and Calculus of Variations

www.esaim-cocv.org

\title{
UNIFORMLY EXPONENTIALLY OR POLYNOMIALLY STABLE APPROXIMATIONS FOR SECOND ORDER EVOLUTION EQUATIONS AND SOME APPLICATIONS
}

\author{
Farah Abdallah ${ }^{1}$, Serge Nicaise ${ }^{2}$, Julie Valein ${ }^{3}$ and Ali Wehbe ${ }^{4}$
}

\begin{abstract}
In this paper, we consider the approximation of second order evolution equations. It is well known that the approximated system by finite element or finite difference is not uniformly exponentially or polynomially stable with respect to the discretization parameter, even if the continuous system has this property. Our goal is to damp the spurious high frequency modes by introducing numerical viscosity terms in the approximation scheme. With these viscosity terms, we show the exponential or polynomial decay of the discrete scheme when the continuous problem has such a decay and when the spectrum of the spatial operator associated with the undamped problem satisfies the generalized gap condition. By using the Trotter-Kato Theorem, we further show the convergence of the discrete solution to the continuous one. Some illustrative examples are also presented.
\end{abstract}

Mathematics Subject Classification. 65M60, 35L05, 35L15.

Received December 21, 2011. Revised May 7, 2012.

Published online June 3, 2013.

\section{INTRODUCTION AND MAIN RESULTS}

Let $H$ be a complex Hilbert space with norm and inner product denoted respectively by $\|\cdot\|$ and $(.,$.$) . Let$ $A: \mathcal{D}(A) \rightarrow H$ be a densely defined self-adjoint and positive operator with a compact inverse in $H$. Let $V=\mathcal{D}\left(A^{\frac{1}{2}}\right)$ be the domain of $A^{\frac{1}{2}}$. Denote by $\mathcal{D}\left(A^{\frac{1}{2}}\right)^{\prime}$ the dual space of $\mathcal{D}\left(A^{\frac{1}{2}}\right)$ obtained by means of the inner product in $H$.

Furthermore, let $U$ be a complex Hilbert space (which will be identified to its dual space) with norm and inner product denoted respectively by $\|\cdot\|_{U}$ and $(., .)_{U}$ and let $B \in \mathcal{L}(U, H)$. We consider the closed loop system

$$
\begin{gathered}
\ddot{\omega}(t)+A \omega(t)+B B^{*} \dot{\omega}(t)=0, \\
\omega(0)=\omega_{0}, \dot{\omega}(0)=\omega_{1},
\end{gathered}
$$

Keywords and phrases. Stability, wave equation, numerical approximations.

1 Université de Valenciennes et du Hainaut Cambrésis, LAMAV, FR CNRS 2956, Institut des Sciences et Techniques of Valenciennes, 59313 Valenciennes Cedex 9, France. The Ph.D. studies of F. Abdallah are financed by the association Azm and Saadé. Farah.Abdallah@meletu.univ-valenciennes.fr

2 Université de Valenciennes et du Hainaut Cambrésis, LAMAV, FR CNRS 2956, Institut des Sciences et Techniques of Valenciennes, 59313 Valenciennes Cedex 9, France. Serge.Nicaise@univ-valenciennes.fr

3 Institut Elie Cartan Nancy (IECN), Nancy-Université \& INRIA (Project-Team CORIDA), 54506 Vandoeuvre-lès-Nancy Cedex France. Julie.Valein@iecn.u-nancy.fr

4 Université Libanaise, Ecole Doctorale des Sciences et de Technologie, Hadath, Beyrouth, Liban. ali.wehbe@ul.edu.lb 
where $t \in[0, \infty)$ represents the time, $\omega:[0, \infty) \rightarrow H$ is the state of the system. Most of the linear equations modeling the vibrations of elastic structures with feedback control (corresponding to collocated actuators and sensors) can be written in the form (1.1), where $\omega$ represents the displacement field.

We define the energy of system (1.1) at time $t$ by

$$
E(t)=\frac{1}{2}\left(\|\dot{\omega}(t)\|^{2}+\left\|A^{\frac{1}{2}} \omega(t)\right\|^{2}\right) .
$$

Simple formal calculations give

$$
E(0)-E(t)=\int_{0}^{t}\left(B B^{*} \dot{\omega}(s), \dot{\omega}(s)\right) \mathrm{d} s, \quad \forall t \geq 0 .
$$

This obviously means that the energy is non-increasing.

In many applications, the system (1.1) is approximated by finite dimensional systems but usually if the continuous system is exponentially or polynomially stable, the discrete ones do no more inherit of this property due to spurious high frequency modes. Several remedies have been proposed and analyzed to overcome this difficulties. Let us quote the Tychonoff regularization [18,19,31,34], a bi-grid algorithm [16,28], a mixed finite element method $[6,10,11,17,27]$, or filtering the high frequencies $[22,25,35]$ (both methods providing good numerical results).

As in [31,34] our goal is to damp the spurious high frequency modes by introducing a numerical viscosity in the approximation schemes. Though our paper is inspired from [31], it differs from that paper on the following points:

(i) Contrary to [31] where the standard gap condition is required, we only assume that the spectrum of the operator $A^{1 / 2}$ satisfies the generalized gap condition, allowing to treat more general concrete systems;

(ii) we analyze the polynomial decay of the discrete schemes when the continuous problem has such a decay;

(iii) we prove a result about uniform polynomial stability for a family of semigroups of operators;

(iv) by using a general version of the Trotter-Kato Theorem proved in [23], we show that the discrete solution tends to the solution of (1.1) as the discretization parameter goes to zero and if the discrete initial data are well chosen.

Before stating our main results, let us introduce some notations and assumptions.

We denote by $\|\cdot\|_{V}$ the norm

$$
\|\varphi\|_{V}=\sqrt{\left(A^{\frac{1}{2}} \varphi, A^{\frac{1}{2}} \varphi\right)}, \forall \varphi \in V
$$

Remark that

$$
\|\varphi\|_{V}=\sqrt{(A \varphi, \varphi)}, \forall \varphi \in \mathcal{D}(A) .
$$

We now assume that $\left(V_{h}\right)_{h>0}$ is a sequence of finite dimensional subspaces of $\mathcal{D}\left(A^{\frac{1}{2}}\right)$. The inner product in $V_{h}$ is the restriction of the inner product of $H$ and it is still denoted by (.,.) (since $V_{h}$ can be seen as a subspace of $H)$. We define the operator $A_{h}: V_{h} \rightarrow V_{h}$ by

$$
\left(A_{h} \varphi_{h}, \psi_{h}\right)=\left(A^{\frac{1}{2}} \varphi_{h}, A^{\frac{1}{2}} \psi_{h}\right), \forall \varphi_{h}, \psi_{h} \in V_{h} .
$$

Let $a(.,$.$) be the sesquilinear form on V_{h} \times V_{h}$ defined by

$$
a\left(\varphi_{h}, \psi_{h}\right)=\left(A^{\frac{1}{2}} \varphi_{h}, A^{\frac{1}{2}} \psi_{h}\right), \forall\left(\varphi_{h}, \psi_{h}\right) \in V_{h} \times V_{h} .
$$

We also define the operators $B_{h}: U \rightarrow V_{h}$ by

$$
B_{h} u=j_{h} B u, \quad \forall u \in U,
$$

where $j_{h}$ is the orthogonal projection of $H$ into $V_{h}$ with respect to the inner product in $H$. 
The adjoint $B_{h}^{*}$ of $B_{h}$ is then given by the relation

$$
B_{h}^{*} \varphi_{h}=B^{*} \varphi_{h}, \quad \forall \varphi_{h} \in V_{h} .
$$

We also suppose that the family of spaces $\left(V_{h}\right)_{h}$ approximates the space $V=\mathcal{D}\left(A^{\frac{1}{2}}\right)$. More precisely, if $\pi_{h}$ denotes the orthogonal projection of $V=\mathcal{D}\left(A^{\frac{1}{2}}\right)$ onto $V_{h}$, we suppose that there exist $\theta>0, h^{*}>0$ and $C_{0}>0$ such that, for all $h \in\left(0, h^{*}\right)$, we have:

$$
\begin{gathered}
\left\|\pi_{h} \varphi-\varphi\right\|_{V} \leq C_{0} h^{\theta}\|A \varphi\|, \forall \varphi \in \mathcal{D}(A), \\
\left\|\pi_{h} \varphi-\varphi\right\| \leq C_{0} h^{2 \theta}\|A \varphi\|, \forall \varphi \in \mathcal{D}(A) .
\end{gathered}
$$

Assumptions (1.5) and (1.6) are, in particular, satisfied in the case of standard finite element approximations of Sobolev spaces.

Denote by $\left\{\lambda_{k}\right\}_{k \geq 1}$ the set of eigenvalues of $A^{\frac{1}{2}}$ counted with their multiplicities (i.e. we repeat the eigenvalues according to their multiplicities). We further rewrite the sequence of eigenvalues $\left\{\lambda_{k}\right\}_{k \geq 1}$ as follows:

$$
\lambda_{k_{1}}<\lambda_{k_{2}}<\ldots<\lambda_{k_{i}}<\ldots
$$

where $k_{1}=1, k_{2}$ is the lowest index of the second distinct eigenvalue, $k_{3}$ is the lowest index of the third distinct eigenvalue, etc. For all $i \in \mathbb{N}^{*}$, let $l_{i}$ be the multiplicity of the eigenvalue $\lambda_{k_{i}}$, i.e.

$$
\lambda_{k_{i-1}}<\lambda_{k_{i}}=\lambda_{k_{i}+1}=\ldots=\lambda_{k_{i}+l_{i}-1}<\lambda_{k_{i}+l_{i}}=\lambda_{k_{i+1}} .
$$

We have $k_{1}=1, k_{2}=1+l_{1}, k_{3}=1+l_{1}+l_{2}$, etc. Let $\left\{\varphi_{k_{i}+j}\right\}_{0 \leq j \leq l_{i}-1}$ be the orthonormal eigenvectors associated with the eigenvalue $\lambda_{k_{i}}$.

Now, we assume that the following generalized gap condition holds:

$$
\exists M \in \mathbb{N}^{*}, \exists \gamma_{0}>0, \forall k \geq 1, \lambda_{k+M}-\lambda_{k} \geq M \gamma_{0} .
$$

Fix a positive real number $\gamma_{0}^{\prime} \leq \gamma_{0}$ and denote by $A_{k}, k=1, \ldots, M$ the set of natural numbers $k_{m}$ satisfying (see for instance [5])

$$
\left\{\begin{array}{l}
\lambda_{k_{m}}-\lambda_{k_{m-1}} \geq \gamma_{0}^{\prime} \\
\lambda_{k_{n}}-\lambda_{k_{n-1}}<\gamma_{0}^{\prime} \\
\lambda_{k_{m+k}}-\lambda_{k_{m+k-1}} \geq \gamma_{0}^{\prime} .
\end{array} \text { for } m+1 \leq n \leq m+k-1,\right.
$$

Then one easily checks that

$$
\left\{k_{m+j}+l \mid k_{m} \in A_{k}, k \in\{1, \ldots, M\}, j \in\{0, \ldots, k-1\}, l \in\left\{0, \ldots, l_{m+j}-1\right\}\right\}=\mathbb{N}^{*} .
$$

Notice that some sets $A_{k}$ may be empty because, for the generalized gap condition, the choice of $M$ takes into account multiple eigenvalues. For $k_{n} \in A_{k}$, we define $B_{k_{n}}=\left(B_{k_{n}, i j}\right)_{1 \leq i, j \leq k}$ the matrix of size $k \times k$ by

$$
B_{k_{n}, i j}= \begin{cases}\prod_{\substack{q=n \\ q \neq n+i-1}}^{n+j-1}\left(\lambda_{k_{n+i-1}}-\lambda_{k_{q}}\right)^{-1} & \text { if } \quad i \leq j,(i, j) \neq(1,1), \\ 1 & \text { if } \quad(i, j)=(1,1), \\ 0 & \text { else. }\end{cases}
$$

More explicitly, we have

$$
B_{k_{n}}=\left(\begin{array}{ccccc}
1 \frac{1}{\lambda_{k_{n}}-\lambda_{k_{n+1}}} & \frac{1}{\left(\lambda_{k_{n}}-\lambda_{k_{n+1}}\right)\left(\lambda_{k_{n}}-\lambda_{k_{n+2}}\right)} & \cdots & \frac{1}{\left(\lambda_{k_{n}}-\lambda_{k_{n+1}}\right) \cdots\left(\lambda_{k_{n}}-\lambda_{k_{n+k-1}}\right)} \\
0 \frac{1}{\lambda_{k_{n}+1}-\lambda_{k_{n}}} & \frac{1}{\left(\lambda_{k_{n+1}}-\lambda_{k_{n}}\right)\left(\lambda_{k_{n+1}}-\lambda_{k_{n+2}}\right)} & \cdots & \frac{1}{\left(\lambda_{k_{n+1}}-\lambda_{k_{n}}\right) \cdots\left(\lambda_{k_{n+1}}-\lambda_{k_{n+k-1}}\right)} \\
0 & 0 & \frac{1}{\left(\lambda_{k_{n+2}}-\lambda_{k_{n}}\right)\left(\lambda_{k_{n+2}}-\lambda_{k_{n+1}}\right)} & \cdots & \frac{1}{\left(\lambda_{k_{n+2}}-\lambda_{k_{n}}\right) \cdots\left(\lambda_{k_{n+2}}-\lambda_{k_{n+k-1}}\right)} \\
\vdots & \vdots & & \ddots & \vdots \\
0 & 0 & 0 & \cdots & \frac{1}{\left(\lambda_{k_{n+k-1}}-\lambda_{k_{n}}\right) \cdots\left(\lambda_{k_{n+k-1}}-\lambda_{k_{n+k-2}}\right)}
\end{array}\right) .
$$


Lemma 1.1. The inverse matrix of $B_{k_{n}}$ is given by

$$
B_{k_{n}, i j}^{-1}= \begin{cases}\prod_{q=n}^{n+i-2}\left(\lambda_{k_{n+j-1}}-\lambda_{k_{q}}\right) & \text { if } \quad i \leq j, i \neq 1 \\ 1 & \text { if } i=1 \\ 0 & \text { else, }\end{cases}
$$

that is to say

$$
B_{k_{n}}^{-1}=\left(\begin{array}{cccc}
1 & 1 & \cdots & 1 \\
0 & \left(\lambda_{k_{n+1}}-\lambda_{k_{n}}\right) & \cdots & \left(\lambda_{k_{n+k-1}}-\lambda_{k_{n}}\right) \\
0 & 0 & \cdots & \left(\lambda_{k_{n+k-1}}-\lambda_{k_{n}}\right)\left(\lambda_{k_{n+k-1}}-\lambda_{k_{n+1}}\right) \\
\vdots & \vdots & \ddots & \vdots \\
0 & 0 & \cdots & \left(\lambda_{k_{n+k-1}}-\lambda_{k_{n}}\right) \cdots\left(\lambda_{k_{n+k-1}}-\lambda_{k_{n+k-2}}\right)
\end{array}\right)
$$

and therefore

$$
B_{k_{n}}^{-1} \rightarrow\left(\begin{array}{cccc}
1 & 1 & \cdots & 1 \\
0 & 0 & \cdots & 0 \\
\vdots & \ddots & \vdots \\
0 & \cdots & 0
\end{array}\right), \text { when } n \rightarrow+\infty
$$

Proof. The form of $B_{k_{n}}^{-1}$ is obtained by induction on the size $k$ of $B_{k_{n}}$. The generalized gap condition (1.7) implies that $\lambda_{k_{n+j}}-\lambda_{k_{n}} \rightarrow 0$ as $n \rightarrow+\infty, \forall 0 \leq j \leq k-1$. This leads to the convergence of $B_{k_{n}}^{-1}$.

Now, for $k_{n} \in A_{k}$, we define the matrix $\Phi_{k_{n}}$ with coefficients in $U$ and size $k \times L_{n}$, where $L_{n}=\sum_{i=1}^{k} l_{n+i-1}$, as follows: for all $i=1, \ldots, k$, we set

$$
\left(\Phi_{k_{n}}\right)_{i j}= \begin{cases}B^{*} \varphi_{k_{n+i-1}+j-L_{n, i-1}-1} & \text { if } L_{n, i-1}<j \leq L_{n, i} \\ 0 & \text { else }\end{cases}
$$

where

$$
L_{n, 0}=0, \quad L_{n, i}=\sum_{i^{\prime}=1}^{i} l_{n+i^{\prime}-1} \text { for } i \geq 1
$$

For a vector $c=\left(c_{l}\right)_{l=1}^{m}$ in $U^{m}$, we set $\|c\|_{U, 2}$ its norm in $U^{m}$ defined by

$$
\|c\|_{U, 2}^{2}=\sum_{l=1}^{m}\left\|c_{l}\right\|_{U}^{2}
$$

In this paper, we prove two results. The first result gives a necessary and sufficient condition to have the exponential stability of the family of systems

$$
\begin{gathered}
\ddot{\omega}_{h}(t)+A_{h} \omega_{h}(t)+B_{h} B_{h}^{*} \dot{\omega}_{h}(t)+h^{\theta} A_{h} \dot{\omega}_{h}(t)=0 \\
\omega_{h}(0)=\omega_{0 h} \in V_{h}, \dot{\omega}_{h}(0)=\omega_{1 h} \in V_{h},
\end{gathered}
$$

in the absence of the standard gap condition assumed in [31]. Here and below $\omega_{0 h}$ (resp. $\omega_{1 h}$ ) is an approximation of $\omega_{0}$ (resp. $\left.\omega_{1}\right)$ in $V_{h}$. For that purpose, we need to make the following assumption

$$
\exists \alpha_{0}>0, \forall k \in\{1, \ldots, M\}, \forall k_{n} \in A_{k}, \forall C \in \mathbb{R}^{L_{n}},\left\|B_{k_{n}}^{-1} \Phi_{k_{n}} C\right\|_{U, 2} \geq \alpha_{0}\|C\|_{2},
$$

where $\|.\|_{2}$ is the euclidian norm. The first main result is the following 
Theorem 1.2. Suppose that the generalized gap condition (1.7) and the assumption (1.10) are verified. Assume that the family of subspaces $\left(V_{h}\right)$ satisfies (1.5) and (1.6). Then the family of systems (1.9) is uniformly exponentially stable, in the sense that there exist constants $M, \alpha, h^{*}>0$ (independent of $h, \omega_{0 h}, \omega_{1 h}$ ) such that for all $h \in\left(0, h^{*}\right):$

$$
\left\|\dot{\omega}_{h}(t)\right\|^{2}+a\left(\omega_{h}(t), \omega_{h}(t)\right) \leq M \mathrm{e}^{-\alpha t}\left(\left\|\omega_{1 h}\right\|^{2}+a\left(\omega_{0 h}, \omega_{0 h}\right)\right), \forall t \geq 0 .
$$

Remark 1.3. If the standard gap condition

$$
\exists \gamma_{0}>0, \forall n \geq 1, \lambda_{k_{n+1}}-\lambda_{k_{n}} \geq \gamma_{0}
$$

holds, then $A_{1}=\mathbb{N}^{*}$ and $B_{1}=1$. In this case, the assumption (1.10) becomes

$$
\exists \alpha_{0}>0, \forall k_{n} \geq 1, \forall C \in \mathbb{R}^{L_{n}},\left\|\Phi_{k_{n}} C\right\|_{U} \geq \alpha_{0}\|C\|_{2} .
$$

Moreover, if the standard gap condition (1.11) holds and if the eigenvalues are simple, the assumption (1.10) becomes

$$
\exists \alpha_{0}>0, \forall k \geq 1,\left\|B^{*} \varphi_{k}\right\|_{U} \geq \alpha_{0} .
$$

These assumptions are assumed in [31].

Remark 1.4. Note that Theorem 1.2 is the discrete counterpart of the exponential decay of the solution of the continuous problem (1.1) under the assumptions (1.7) and (1.10), which follows Theorem 2.2 of [3] (see also [29]). Note that the assumption (H) from [3] here holds since $A$ is a positive selfadjoint operator with a compact resolvent and $B$ is bounded.

Remark 1.5. The uniform exponential stability of the family of systems (1.9) has been already proved in Theorem 7.1 of [14] without any assumption on the spectrum of $A$ and the dimension of the space. The proof of this theorem is based on decoupling of low and high frequencies. More precisely, the author combines a uniform observability estimate for filtered initial data corresponding to low frequencies (see [14], Thm. 1.3) together with a result of [15]. Indeed, in [15], after adding the numerical viscosity term, another uniform observability estimate is obtained for the high frequency components. The two established observability inequalities yield the uniform exponential decay of (1.9).

If the condition (1.10) is not satisfied, we may look at a weaker version. Namely if we assume that

$$
\exists l \in \mathbb{N}^{*}, \exists \alpha_{0}>0, \forall k \in\{1, \ldots, M\}, \forall k_{n} \in A_{k}, \forall C \in \mathbb{R}^{L_{n}},\left\|B_{k_{n}}^{-1} \Phi_{k_{n}} C\right\|_{U, 2} \geq \frac{\alpha_{0}}{\lambda_{k_{n}}^{l}}\|C\|_{2},
$$

then we will obtain a polynomial stability for the family of systems

$$
\begin{gathered}
\ddot{\omega}_{h}(t)+\left(1+h^{\theta}\right)^{-2}\left(I+h^{\theta} A_{h}^{\frac{l}{2}}\right)^{2} A_{h} \omega_{h}(t)+\left(I+h^{\theta} A_{h}^{\frac{l}{2}}\right)\left(B_{h} B_{h}^{*}+h^{\theta} A_{h}^{1+\frac{l}{2}}\right)\left(I+h^{\theta} A_{h}^{\frac{l}{2}}\right)^{-1} \dot{\omega}_{h}(t)=0, \\
\omega_{h}(0)=\omega_{0 h} \in V_{h}, \dot{\omega}_{h}(0)=\left(1+h^{\theta}\right)^{-1}\left(I+h^{\theta} A_{h}^{\frac{l}{2}}\right) \omega_{1 h} \in V_{h} .
\end{gathered}
$$

The structure of the above discrete system has been inspired from the one introduced in [31] for the exponential stability case where the authors have used system (1.9) corresponding to $l=0$. In both cases, this choice is motivated by the corresponding observability estimates. The numerical viscosity term $\left(I+h^{\theta} A_{h}^{\frac{l}{2}}\right)\left(B_{h} B_{h}^{*}+\right.$ $\left.h^{\theta} A_{h}^{1+\frac{l}{2}}\right)\left(I+h^{\theta} A_{h}^{\frac{l}{2}}\right)^{-1} \dot{\omega}_{h}(t)$ is added to damp the high frequency modes and as the set of high frequency modes is larger in the polynomial case, the viscosity term is naturally stronger. In the case $l \geq 2$ the powers of $\left(I+h^{\theta} A_{h}^{\frac{l}{2}}\right)$ have been added to guarantee the boundedness of the resolvent of $\tilde{A}_{l, h}$ (defined below) near zero. The question of the optimality of these viscosity terms remains open.

The second main result of our paper is the following one. 
Theorem 1.6. Suppose that the generalized gap condition (1.7) and the Assumption (1.13) are verified with $l \in \mathbb{N}^{*}$ even. Assume that the family of subspaces $\left(V_{h}\right)$ satisfies (1.5) and (1.6). Then the family of systems (1.14) is uniformly polynomially stable, in the sense that there exist constants $C, h^{*}>0$ (independent of $h, \omega_{0 h}, \omega_{1 h}$ ) such that for all $h \in\left(0, h^{*}\right)$ :

$$
\begin{aligned}
& \left\|\left(I+h^{\theta} A_{h}^{\frac{l}{2}}\right)^{-1} \dot{\omega}_{h}(t)\right\|^{2}+a\left(\omega_{h}(t), \omega_{h}(t)\right) \leq \frac{C}{t^{2}}\left\|\left(\omega_{0 h}, \omega_{1 h}\right)\right\|_{D\left(\tilde{A}_{l, h}^{2 l}\right)}^{2}, \\
& \left\|\left(I+h^{\theta} A_{h}^{\frac{l}{2}}\right)^{-1} \dot{\omega}_{h}(t)\right\|^{2}+a\left(\omega_{h}(t), \omega_{h}(t)\right) \leq \frac{C}{t^{\frac{1}{l}}}\left\|\left(\omega_{0 h}, \omega_{1 h}\right)\right\|_{D\left(\tilde{A}_{l, h}\right)}^{2}, \forall t>0, \forall\left(\omega_{0 h}, \omega_{1 h}\right) \in V_{h} \times V_{h},
\end{aligned}
$$

where for $q \in \mathbb{N}^{*},\|\cdot\|_{D\left(\tilde{A}_{l, h}^{q}\right)}$ is the graph norm of the matrix operator $\tilde{A}_{l, h}^{q}$ given in (4.1) of Section 4 below.

For a technical reason, we assume $l$ to be even (see Lem. 4.4). If (1.13) holds for $l$ odd, then it is also true for $l+1$ and we can apply the previous result with $l+1$.

Remark 1.7. If the standard gap condition (1.11) holds, the Assumption (1.13) becomes

$$
\exists l \in \mathbb{N}^{*}, \exists \alpha_{0}>0, \forall k_{n} \geq 1, \forall C \in \mathbb{R}^{L_{n}},\left\|\Phi_{k_{n}} C\right\|_{U} \geq \frac{\alpha_{0}}{\lambda_{k_{n}}^{l}}\|C\|_{2} .
$$

Moreover, if the standard gap condition (1.11) holds and if the eigenvalues are simple, the Assumption (1.13) becomes

$$
\exists l \in \mathbb{N}^{*}, \exists \alpha_{0}>0, \forall k \geq 1,\left\|B^{*} \varphi_{k}\right\|_{U} \geq \frac{\alpha_{0}}{\lambda_{k}^{l}} .
$$

Remark 1.8. As before, Theorem 1.6 is the discrete counterpart of the polynomial decay of the solution of the continuous problem (1.1) under the Assumptions (1.7) and (1.13), that follows from Theorem 2.4 of [3] (see also [29]).

The paper is organized as follows: In Section 2, we show that the generalized gap condition and the observability conditions (1.10) and (1.13) remain valid for filtered eigenvalues. Section 3 first recalls a result about uniform exponential stability for a family of semigroup of operators, and then extends such a result to the case of uniform polynomial stability. Some technical lemmas are proved in Section 4 . Sections 5 and 6 are devoted to the proof of Theorem 1.2 and 1.6 respectively. In Section 7, we show that the solution $\omega_{h}$ (resp. $\left.\left(I+h^{\theta} A_{h}^{\frac{l}{2}}\right)^{-1} \dot{\omega}_{h}\right)$ tends to $\omega$, the solution of (1.1), (resp. $\dot{\omega}$ ) in $V$ (resp. in $H$ ) as $h$ goes to zero and if the discrete initial data are well chosen. Finally, we illustrate our results by presenting different examples in Section 8.

\section{Spectral AnAlysis of the Discretized problem}

The eigenvalue problem of the discretized problem is the following one: find $\left.\lambda_{k, h} \in\right] 0,+\infty\left[, \varphi_{k, h} \in V_{h}\right.$, such that

$$
a\left(\varphi_{k, h}, \psi_{h}\right)=\lambda_{k, h}^{2}\left(\varphi_{k, h}, \psi_{h}\right), \forall \psi_{h} \in V_{h} .
$$

Let $N(h)$ be the dimension of $V_{h}$. We denote by $\left\{\lambda_{k, h}^{2}\right\}_{1 \leq k \leq N(h)}$ the set of eigenvalues of (2.1) counted with their multiplicities. Let $\left\{\varphi_{k, h}\right\}_{1 \leq k \leq N(h)}$ be the orthonormal eigenvectors associated with the eigenvalue $\lambda_{k, h}^{2}$.

In this Section, we show that the generalized gap condition (1.7) and the observability conditions (1.10) and (1.13) still hold for the approximate problem (uniformly in $h$ ), provided that we consider only "low frequencies". More precisely, we first have the following result:

Proposition 2.1. Suppose that the generalized gap condition (1.7) and the Assumption (1.10) are verified. Then, there exist two constants $\epsilon>0$ and $h^{*}>0$, such that, for all $0<h<h^{*}$ and for all $k \in\{1, \ldots, N(h)\}$ satisfying

$$
h^{\theta} \lambda_{k}^{2} \leq \epsilon
$$


we have

$$
\exists M \in \mathbb{N}^{*}, \exists \gamma>0, \lambda_{k+M, h}-\lambda_{k, h} \geq M \gamma
$$

and

$$
\exists \alpha>0, \forall p \in\{1, \ldots, M\}, \forall k_{n} \in A_{p, h}, \forall C \in \mathbb{R}^{L_{n}},\left\|B_{k_{n}}^{-1} \Phi_{k_{n}, h} C\right\|_{U, 2} \geq \alpha\|C\|_{2},
$$

where $\alpha$ is independent of $h$, and where the matrix $\Phi_{k_{n}, h} \in \mathcal{M}_{p, L_{n}}(U)$, with coefficients in $U$, is defined as follows: for all $i=1, \ldots, p$, we set

$$
\left(\Phi_{k_{n}, h}\right)_{i j}= \begin{cases}B_{h}^{*} \varphi_{k_{n+i-1}+j-L_{n, i-1}-1, h} & \text { if } L_{n, i-1}<j \leq L_{n, i}, \\ 0 & \text { else, }\end{cases}
$$

where $L_{n, i-1}$ is defined by (1.8) and

$$
A_{p, h}=\left\{k_{n} \in A_{p} \text { satisfying }(2.2) \text { and s.t. } k_{n+p-1}+l_{n+p-1}-1 \leq N(h)\right\} .
$$

For the proof of this proposition, we need a result proved by Babuska and Osborn in [4]. For that purpose, we introduce $\epsilon_{h}(n, j)$ such that

$$
\epsilon_{h}(n, j)=\inf _{\varphi \in M_{j}\left(\lambda_{k_{n}}\right)} \inf _{v_{h} \in V_{h}}\left\|\varphi-v_{h}\right\|_{V}
$$

where $M_{j}\left(\lambda_{k_{n}}\right)=\left\{\varphi \in M\left(\lambda_{k_{n}}\right): a\left(\varphi, \varphi_{k_{n}, h}\right)=\ldots=a\left(\varphi, \varphi_{k_{n}+j-2, h}\right)=0\right\}$ and $M\left(\lambda_{k_{n}}\right)=$ $\left\{\varphi: \varphi\right.$ is an eigenvector of $A^{\frac{1}{2}}$ corresponding to $\left.\lambda_{k_{n}},\|\varphi\|=1\right\}$. The restrictions $a\left(\varphi, \varphi_{k_{n}, h}\right)=\ldots=$ $a\left(\varphi, \varphi_{k_{n}+j-2, h}\right)=0$ are not imposed if $j=1$. Then, we have the following estimate about the eigenvalue and eigenvector errors for the Galerkin method in terms of the approximability quantities $\epsilon_{h}(n, j)$.

Theorem 2.2. There are positive constants $C$ and $h_{0}$ such that

$$
\lambda_{k_{n}+j, h}-\lambda_{k_{n}+j} \leq C \epsilon_{h}^{2}(n, j), \quad \forall 0<h \leq h_{0}, j=0, \ldots, l_{n}-1, k_{n}+j \leq N(h), n \in \mathbb{N}^{*}
$$

and such that the eigenvectors $\left\{\varphi_{k_{n}+j}\right\}_{0 \leq j \leq l_{n}-1}$ of $A^{\frac{1}{2}}$ can be chosen so that

$$
\left\|\varphi_{k_{n}+j, h}-\varphi_{k_{n}+j}\right\|_{V} \leq C \epsilon_{h}(n, j), \quad \forall 0<h \leq h_{0}, j=0, \ldots, l_{n}-1, k_{n}+j \leq N(h), n \in \mathbb{N}^{*} .
$$

This result is proved by Babuska and Osborn in [4], p. 702. because

$$
\lambda_{k_{n}+j, h}^{2}-\lambda_{k_{n}+j}^{2}=\left(\lambda_{k_{n}+j, h}-\lambda_{k_{n}+j}\right)\left(\lambda_{k_{n}+j, h}+\lambda_{k_{n}+j}\right) \geq 2 \lambda_{1}\left(\lambda_{k_{n}+j, h}-\lambda_{k_{n}+j}\right) .
$$

Remark 2.3. Notice that for every $\varphi \in M_{j}\left(\lambda_{k_{n}}\right)$ we have

$$
\begin{aligned}
\epsilon_{h}(n, j) & \leq \inf _{v_{h} \in V_{h}}\left\|\varphi-v_{h}\right\|_{V} \\
& \leq C_{0} h^{\theta}\|A \varphi\| \text { by }(1.5) \\
& \leq C_{0} h^{\theta} \lambda_{k_{n}}^{2}\|\varphi\|=C_{0} h^{\theta} \lambda_{k_{n}+j}^{2} .
\end{aligned}
$$

Proof of Proposition 2.1. We begin with the proof of the generalized gap condition for the approximate eigenvalues $\lambda_{k, h}$. First, we use the Min-Max principle (see [32]) to obtain

$$
\lambda_{k} \leq \lambda_{k, h}, \forall k \in\{1, \ldots, N(h)\} .
$$

Second, we use the estimates (2.5) and (2.7) and we have

$$
\lambda_{k, h} \leq \lambda_{k}+C\left(C_{0} h^{\theta} \lambda_{k}^{2}\right)^{2} \leq \lambda_{k}+C\left(C_{0} \epsilon\right)^{2} \leq \lambda_{k}+C C_{0}^{2} \epsilon,
$$

for all $k \in\{1, \ldots, N(h)\}$ verifying $(2.2)$ and $\epsilon \leq 1$. Therefore, we may write

$$
\lambda_{k+M, h}-\lambda_{k, h} \geq \lambda_{k+M}-\lambda_{k}-C C_{0}^{2} \epsilon \geq M \gamma_{0}-C C_{0}^{2} \epsilon \geq M \frac{\gamma_{0}}{2}=: M \gamma
$$

for all $k \in\{1, \ldots, N(h)\}$ satisfying $(2.2)$ and for $\epsilon \leq \frac{M \gamma_{0}}{2 C C_{0}^{2}}$. 
Now, we prove the estimate (2.4) which is the approximated version of (1.10). Notice that

$$
\begin{aligned}
\left\|\Phi_{k_{n}, h}-\Phi_{k_{n}}\right\|_{U} & \leq C_{i=0, \ldots, p-1} \sum_{j=0} \sum^{l_{n+i}-1}\left\|B^{*} \varphi_{k_{n+i}+j, h}-B^{*} \varphi_{k_{n+i}+j}\right\|_{U} \\
& \leq C \max _{i=0, \ldots, p-1} \sum_{j=0}^{l_{n+i}-1}\left\|B^{*}\right\|_{\mathcal{L}(H, U)}\left\|\varphi_{k_{n+i}+j, h}-\varphi_{k_{n+i}+j}\right\|^{l_{n+i}-1}\left\|B^{*}\right\|_{\mathcal{L}(H, U)}\left\|\varphi_{k_{n+i}+j, h}-\varphi_{k_{n+i}+j}\right\|_{V} \\
& \leq C_{i=0, \ldots, p-1} \sum_{j=0} \leq C_{i=0, \ldots, p-1} \sum_{j=0}^{\max _{n+i}-1} \epsilon_{h}(n+i, j) \text { by }(2.6) \\
& \leq C_{i=0, \ldots, p-1} \sum_{j=0}^{\operatorname{lax}_{n+i-1}} h^{\theta} \lambda_{k_{n+i}+j}^{2} \text { by }(2.7) .
\end{aligned}
$$

Thus, by (2.2), we get

$$
\left\|\Phi_{k_{n}, h}-\Phi_{k_{n}}\right\|_{U} \leq C \epsilon .
$$

Therefore the triangular inequality leads to

$$
\begin{aligned}
\left\|B_{k_{n}}^{-1} \Phi_{k_{n}, h} C\right\|_{U, 2} & =\left\|B_{k_{n}}^{-1} \Phi_{k_{n}} C+B_{k_{n}}^{-1}\left(\Phi_{k_{n}, h}-\Phi_{k_{n}}\right) C\right\|_{U, 2} \\
& \geq\left\|B_{k_{n}}^{-1} \Phi_{k_{n}} C\right\|_{U, 2}-\left\|B_{k_{n}}^{-1}\left(\Phi_{k_{n}, h}-\Phi_{k_{n}}\right) C\right\|_{U, 2} \\
& \geq \alpha_{0}\|C\|_{2}-\left\|B_{k_{n}}^{-1}\left(\Phi_{k_{n}, h}-\Phi_{k_{n}}\right) C\right\|_{U, 2}
\end{aligned}
$$

by (1.10). But, as $B_{k_{n}}^{-1}=\left(\begin{array}{cccc}1 & 1 & \cdots & 1 \\ 0 & 0 & \cdots & 0 \\ \vdots & \ddots & \vdots \\ 0 & \cdots & 0\end{array}\right)+R_{k_{n}}$, with $R_{k_{n}} \rightarrow 0$, when $k_{n} \rightarrow+\infty$ (see Lem. 1.1), we obtain

$$
\begin{aligned}
& \left\|B_{k_{n}}^{-1}\left(\Phi_{k_{n}, h}-\Phi_{k_{n}}\right) C\right\|_{U, 2} \leq\left\|\left(\begin{array}{cccc}
1 & 1 & \cdots & 1 \\
0 & 0 & \cdots & 0 \\
\vdots & \ddots & \vdots \\
0 & \cdots & 0
\end{array}\right)\left(\Phi_{k_{n}, h}-\Phi_{k_{n}}\right) C\right\|_{U, 2} \\
& +\left\|R_{k_{n}}\left(\Phi_{k_{n}, h}-\Phi_{k_{n}}\right) C\right\|_{U, 2} \\
& \leq C\left\|\Phi_{k_{n}, h}-\Phi_{k_{n}}\right\|_{U}\|C\|_{2}+\eta_{n}\left\|\Phi_{k_{n}, h}-\Phi_{k_{n}}\right\|_{U}\|C\|_{2} \\
& \leq C \epsilon\left(1+\eta_{n}\right)\|C\|_{2} \text {, }
\end{aligned}
$$

where $\eta_{n}=\left\|R_{k_{n}}\right\| \rightarrow 0$. Thus

$$
\left\|B_{k_{n}}^{-1} \Phi_{k_{n}, h} C\right\|_{U, 2} \geq\left(\alpha_{0}-C \epsilon\left(1+\eta_{n}\right)\right)\|C\|_{2} \geq \frac{\alpha_{0}}{2}\|C\|_{2}
$$

for $\epsilon \leq \frac{\alpha_{0}}{2 C\left(1+\max _{n}\left(1+\eta_{n}\right)\right)}$.

For the polynomial stability, we have the same kind of result, but more filtering is necessary in order to have the discrete counterpart of the observability condition (1.13) (uniformly in $h$ ).

Proposition 2.4. Suppose that the generalized gap condition (1.7) and the Assumption (1.13) are verified. Then, there exist two constants $\epsilon>0$ and $h^{*}>0$, such that, for all $0<h<h^{*}$ and for all $k \in\{1, \ldots, N(h)\}$, satisfying

$$
h^{\theta} \lambda_{k}^{2} \leq \frac{\epsilon}{\lambda_{k}^{l}}
$$


we have (2.3) and

$$
\exists \alpha>0, \forall p \in\{1, \ldots, M\}, \forall k_{n} \in A_{p, h}^{(l)}, \forall C \in \mathbb{R}^{L_{n}},\left\|B_{k_{n}}^{-1} \Phi_{k_{n}, h} C\right\|_{U, 2} \geq \frac{\alpha}{\lambda_{k_{n}}^{l}}\|C\|_{2},
$$

where $A_{p, h}^{(l)}=\left\{k_{n} \in A_{p}\right.$ satisfying (2.12) and s.t. $\left.k_{n+p-1}+l_{n+p-1}-1 \leq N(h)\right\}$.

Proof. The generalized gap condition for the approximate eigenvalues $\lambda_{k, h}$ is a consequence of Proposition 2.1, because $\lambda_{k} \geq \lambda_{1}>0$.

To prove the estimate $(2.13)$ we notice that

$$
\left\|\Phi_{k_{n}, h}-\Phi_{k_{n}}\right\|_{U} \leq C \max _{i=0, \ldots, p-1} \sum_{j=0}^{l_{n+i}-1} h^{\theta} \lambda_{k_{n+i}+j}^{2} \leq C h^{\theta} \lambda_{k_{n+p-1}}^{2} .
$$

Moreover, by the triangular inequality and (1.13), we have

$$
\left\|B_{k_{n}}^{-1} \Phi_{k_{n}, h} C\right\|_{U, 2} \geq \frac{\alpha_{0}}{\lambda_{k_{n}}^{l}}\|C\|_{2}-\left\|B_{k_{n}}^{-1}\left(\Phi_{k_{n}, h}-\Phi_{k_{n}}\right) C\right\|_{U, 2} .
$$

By (2.11) and (2.12), we obtain

$$
\begin{aligned}
\left\|B_{k_{n}}^{-1} \Phi_{k_{n}, h} C\right\|_{U, 2} & \geq\left(\frac{\alpha_{0}}{\lambda_{k_{n}}^{l}}-\frac{C\left(1+\eta_{n}\right) \epsilon}{\lambda_{k_{n}+p-1}^{l}}\right)\|C\|_{2} \\
& \geq\left(\frac{\alpha_{0}}{\lambda_{k_{n}}^{l}}-\frac{C \epsilon}{\lambda_{k_{n}}^{l}+\rho_{n}}\left(1+\eta_{n}\right)\right)\|C\|_{2}, \text { with } \rho_{n}=\lambda_{k_{n+p-1}}^{l}-\lambda_{k_{n}}^{l} \rightarrow 0 \\
& \geq \frac{\alpha}{\lambda_{k_{n}}^{l}}\|C\|_{2}
\end{aligned}
$$

for an appropriate choice of $\epsilon>0$.

\section{UNIFORM STABILITY RESULTS}

\subsection{Exponential stability result}

The Proof of Theorem 1.2 is based on the following result (see Thm. 7.1.3 in [26]):

Theorem 3.1. Let $\left(T_{h}\right)_{h>0}$ be a family of semigroups of contractions on the Hilbert spaces $\left(X_{h}\right)_{h>0}$ and let $\left(\tilde{A}_{h}\right)_{h>0}$ be the corresponding infinitesimal generators. The family $\left(T_{h}\right)_{h>0}$ is uniformly exponentially stable, that is to say there exist constants $M>0, \alpha>0$ (independent of $h \in\left(0, h^{*}\right)$ ) such that

$$
\left\|T_{h}(t)\right\|_{\mathcal{L}\left(X_{h}\right)} \leq M \mathrm{e}^{-\alpha t}, \forall t \geq 0,
$$

if and only if the two following conditions are satisfied:

(i) For all $h \in\left(0, h^{*}\right), i \mathbb{R}$ is contained in the resolvent set $\rho\left(\tilde{A}_{h}\right)$ of $\tilde{A}_{h}$,

(ii) $\sup _{h \in\left(0, h^{*}\right), \omega \in \mathbb{R}}\left\|\left(i \omega-\tilde{A}_{h}\right)^{-1}\right\|_{\mathcal{L}\left(X_{h}\right)}<+\infty$.

\subsection{Polynomial stability result}

The proof of Theorem 1.6 is based on the results presented in this section by adapting the results from [9] and from [24] to obtain the (uniform) polynomial stability of the discretized problem (1.14). Throughout this

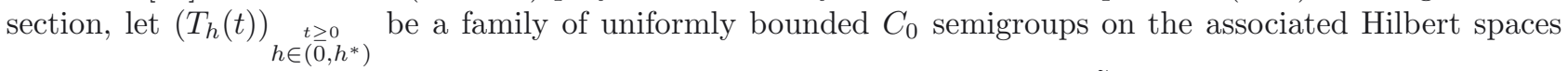
$\left(X_{h}\right)_{h \in\left(0, h^{*}\right)}$ (i.e., $\left.\exists M>0, \forall h \in\left(0, h^{*}\right),\left\|T_{h}(t)\right\|_{\mathcal{L}\left(X_{h}\right)} \leq M\right)$ and let $\left(\tilde{A}_{h}\right)_{h \in\left(0, h^{*}\right)}$ be the corresponding infinitesimal generators.

In the following, for shortness, we denote by $R\left(\lambda, \tilde{A}_{h}\right)$ the resolvent $\left(\lambda-\tilde{A}_{h}\right)^{-1}$; moreover, for any operator mapping $X_{h}$ into $X_{h}$, we skip the index $\mathcal{L}\left(X_{h}\right)$ in its norm, since in the whole section we work in $X_{h}$. 
Definition 3.2. Assuming that

$$
i \mathbb{R} \subseteq \rho\left(\tilde{A}_{h}\right), \quad \forall h \in\left(0, h^{*}\right),
$$

and that for all $m \geq 1$, there exists $c=c(m)>0$ such that

$$
\sup _{\substack{h \in\left(0, h^{*}\right) \\|s| \leq m}}\left\|R\left(i s, \tilde{A}_{h}\right)\right\|_{\mathcal{L}\left(X_{h}\right)} \leq c,
$$

we define the fractional power $\tilde{A}_{h}^{-\alpha}$ for $\alpha>0$ and $h \in\left(0, h^{*}\right)$, according to [2] and [13], as

$$
\tilde{A}_{h}^{-\alpha}=\frac{1}{2 \pi i} \int_{\Gamma} \lambda^{-\alpha}\left(\lambda-\tilde{A}_{h}\right)^{-1} \mathrm{~d} \lambda,
$$

where $\lambda^{-\alpha}=\mathrm{e}^{-\alpha \log \lambda}$ and $\mathbb{R}^{+}$is taken as the cut branch of the complex log function and where the curve $\Gamma=\Gamma_{1} \cup \Gamma_{2}$ is given by

$$
\Gamma=\left\{-\epsilon+t \mathrm{e}^{i \theta}, t \in[0,+\infty)\right\} \cup\left\{-\epsilon-t \mathrm{e}^{-i \theta}, t \in(-\infty, 0]\right\}
$$

for some $\epsilon>0$ small enough independent of $h$ and $\theta$ is a fixed angle in $\left(0, \frac{\pi}{4}\right)$.

Remark 3.3. Throughout this section, whenever $\tilde{A}_{h}^{-\alpha}$ is mentioned, the Assumptions (3.1) and (3.2) are directly taken into consideration since otherwise $\tilde{A}_{h}^{-\alpha}$ is not well defined.

In fact, under the Assumptions (3.1) and (3.2), for all $m>0$ there exists $\epsilon=\epsilon(m)>0$ such that

$$
-\mu+i \beta \in \rho\left(\tilde{A}_{h}\right), \quad \forall h \in\left(0, h^{*}\right), \forall 0 \leq \mu \leq \epsilon, \forall|\beta| \leq m .
$$

Indeed, for all $m>0$ such that $|\beta| \leq m$, we have

$$
\left(-\mu+i \beta-\tilde{A}_{h}\right)^{-1}=\left(i \beta-\tilde{A}_{h}\right)^{-1}\left[I_{h}-\mu\left(i \beta-\tilde{A}_{h}\right)^{-1}\right]^{-1}
$$

and

$$
\left\|\mu\left(i \beta-\tilde{A}_{h}\right)^{-1}\right\| \leq \mu c .
$$

Hence, if $|\beta| \leq m$ and $\mu \leq \epsilon \leq \frac{1}{2 c}$, then $\left(-\mu+i \beta-\tilde{A}_{h}\right)$ is invertible and we have

$$
\left\|\left(-\mu+i \beta-\tilde{A}_{h}\right)^{-1}\right\| \leq 2\left\|\left(i \beta-\tilde{A}_{h}\right)^{-1}\right\| \leq 2 c, \forall h \in\left(0, h^{*}\right) .
$$

We choose $m=\Im\left(-\epsilon+t \mathrm{e}^{i \theta}\right)=\epsilon \tan \theta$ when $\Re\left(-\epsilon+t \mathrm{e}^{i \theta}\right)=0$, i.e. when $t=\frac{\epsilon}{\cos \theta}$. Therefore, by (3.4), Assumptions (3.1) and (3.2) imply that there exists $\epsilon>0$ independent of $h$ such that the curve $\Gamma$ is included in $\rho\left(\tilde{A}_{h}\right)$ for any $h \in\left(0, h^{*}\right)$, and hence $\tilde{A}_{h}^{-\alpha}$ is well defined. In fact, if $\xi \in \Gamma$ such that $\Re \xi>0$, then, by the Hille Yosida theorem, $\xi \in \rho\left(\tilde{A}_{h}\right)$, while if $-\epsilon \leq \xi \leq 0$, then, by $(3.4), \xi \in \rho\left(\tilde{A}_{h}\right)$.

Proposition 3.4. If, in addition to Assumptions (3.1) and (3.2), we have

$$
\sup _{h \in\left(0, h^{*}\right)}\left\|R\left(i s, \tilde{A}_{h}\right)\right\|_{\mathcal{L}\left(X_{h}\right)}=O\left(|s|^{\alpha}\right),|s| \rightarrow \infty
$$

then $\tilde{A}_{h}^{-\alpha}$ is uniformly bounded independently of $h \in\left(0, h^{*}\right)$. 
Proof. We have

$$
\begin{aligned}
\tilde{A}_{h}^{-\alpha}= & \frac{1}{2 \pi i} \int_{0}^{+\infty}\left(-\epsilon+t \mathrm{e}^{i \theta}\right)^{-\alpha}\left(-\epsilon+t \mathrm{e}^{i \theta}-\tilde{A}_{h}\right)^{-1} \mathrm{e}^{i \theta} \mathrm{d} t \\
& +\frac{1}{2 \pi i} \int_{-\infty}^{0}\left(-\epsilon-t \mathrm{e}^{-i \theta}\right)^{-\alpha}\left(-\epsilon-t \mathrm{e}^{-i \theta}-\tilde{A}_{h}\right)^{-1}\left(-\mathrm{e}^{-i \theta}\right) \mathrm{d} t .
\end{aligned}
$$

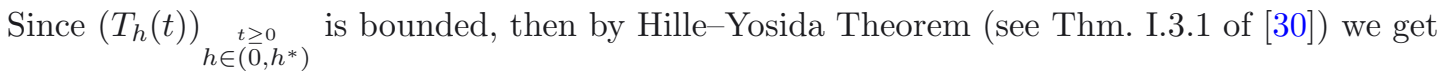

$$
\left\|R\left(\lambda, \tilde{A}_{h}\right)\right\| \leq \frac{M}{\operatorname{Re} \lambda}, \forall \operatorname{Re} \lambda>0 .
$$

For $-\epsilon \leq \operatorname{Re} \lambda \leq 0$, we have $|\Im \lambda| \leq m$ and therefore, by (3.4), we get

$$
\left\|R\left(\lambda, \tilde{A}_{h}\right)\right\| \leq 2 c .
$$

Let $t_{0}>0$ be such that $-\epsilon \leq \operatorname{Re}\left(-\epsilon+t \mathrm{e}^{i \theta}\right) \leq 0, \forall 0 \leq t \leq t_{0}=\frac{\epsilon}{\cos \theta}$ and $\operatorname{Re}\left(-\epsilon+t \mathrm{e}^{i \theta}\right) \geq 0, \forall t \geq t_{0}$ and let $t_{1}=-\frac{\epsilon}{\cos \theta} \leq 0$ be such that $\operatorname{Re}\left(-\epsilon-t \mathrm{e}^{-i \theta}\right) \leq 0, \forall t_{1} \leq t \leq 0$ and $\operatorname{Re}\left(-\epsilon-t \mathrm{e}^{-i \theta}\right) \geq 0, \forall t \leq t_{1}$. Therefore, split the integrals in (3.6) then use (3.4) in case of $0 \leq t \leq t_{0}$ or $t_{1} \leq t \leq 0$; in addition to (3.5) in case of $t \geq t_{0}$ or $t \leq t_{1}$ to get the uniform boundedness of $\tilde{A}_{h}^{-\alpha}$.

The proof of the polynomial stability of $\left(T_{h}(t)\right)_{t \geq 0}$ (see Thm. 3.8 below) is based on the following three lemmas. The first lemma is the discretized version of Lemma 3.2 in [24] and the other ones are the discrete versions of similar results of Lemmas 2.1 and 2.3 in [9].

Lemma 3.5. Let $S=\{\lambda \in \mathbb{C}: a \leq$ Re $\lambda \leq b\}$ be a subset of $\rho\left(\tilde{A}_{h}\right)$ for all $h \in\left(0, h^{*}\right)$ where $0 \leq a<b$. Then if (3.1)-(3.5) are satisfied and if for some positive constants $\alpha$ and $M$ we have

$$
\sup _{\substack{h \in\left(0, h^{*}\right) \\ \lambda \in S}} \frac{\left\|R\left(\lambda, \tilde{A}_{h}\right)\right\|}{1+|\lambda|^{\alpha}} \leq M,
$$

then there exists a constant $c>0$ independent of $h$ such that

$$
\sup _{\substack{h \in\left(0, h^{*}\right) \\ \lambda \in S}}\left\|R\left(\lambda, \tilde{A}_{h}\right) \tilde{A}_{h}^{-\alpha}\right\| \leq c .
$$

Proof. There exists $c>0$ and $\varphi_{0}, 0<\varphi_{0}<\frac{\pi}{2}$, such that

$$
\left|\mu-\mathrm{e}^{i \varphi}\right| \geq c|\mu|, \quad \forall \mu \in \Gamma, \forall \varphi_{0}<|\varphi|<\pi-\varphi_{0}
$$

where the curve $\Gamma$ is given by (3.3).

Since $b$ is finite, choose $N$ large enough such that whenever $\lambda \in S$ and $|\lambda|>N$ we get both $\varphi_{0}<|\arg \lambda|<$ $\pi-\varphi_{0}$ and $\lambda$ does not belong to the sector bounded by the curve $|\lambda| \Gamma=\left\{-\epsilon|\lambda|+t|\lambda| \mathrm{e}^{i \theta}, t \in[0,+\infty)\right\} \cup$ $\left\{-\epsilon|\lambda|-t|\lambda| \mathrm{e}^{-i \theta}, t \in(-\infty, 0]\right\}$.

For all such choice of $\lambda \in S$, we have according to (3.7)

$$
\left|\mu-\mathrm{e}^{\operatorname{iarg} \lambda}\right| \geq c|\mu| \forall \mu \in \Gamma .
$$

Consider the following integral for all $\lambda \in S$ with $|\lambda|>N$

$$
I_{\lambda}=\int_{\Gamma} \frac{\mu^{-\alpha}}{\mu-\lambda} \mathrm{d} \mu .
$$


By the above choice of $\lambda$, we have $\lambda \notin \Gamma$ and $\lambda \notin|\lambda| \Gamma$. Consequently, the integral has no singular points between $\Gamma$ and $|\lambda| \Gamma$. Therefore, by the Cauchy theorem, we have

$$
I_{\lambda}=\int_{|\lambda| \Gamma} \frac{\mu^{-\alpha}}{\mu-\lambda} \mathrm{d} \mu=\frac{1}{|\lambda|^{\alpha}} \int_{\Gamma} \frac{\mu^{-\alpha}}{\mu-\mathrm{e}^{\mathrm{iarg} \lambda}} \mathrm{d} \mu .
$$

Therefore, by (3.8), we get

$$
\left|I_{\lambda}\right| \leq \frac{c}{|\lambda|^{\alpha}}
$$

Now, for $|\lambda|>N$ with $\lambda \in S$, we have by the resolvent identity

$$
\begin{aligned}
R\left(\lambda, \tilde{A}_{h}\right) \tilde{A}_{h}^{-\alpha} & =\frac{1}{2 \pi i} \int_{\Gamma} \mu^{-\alpha} R\left(\lambda, \tilde{A}_{h}\right) R\left(\mu, \tilde{A}_{h}\right) \mathrm{d} \mu \\
& =\frac{1}{2 \pi i} \int_{\Gamma} \frac{\mu^{-\alpha}}{\mu-\lambda} R\left(\lambda, \tilde{A}_{h}\right) \mathrm{d} \mu-\frac{1}{2 \pi i} \int_{\Gamma} \frac{\mu^{-\alpha}}{\mu-\lambda} R\left(\mu, \tilde{A}_{h}\right) \mathrm{d} \mu \\
& =\frac{1}{2 \pi i} I_{\lambda} R\left(\lambda, \tilde{A}_{h}\right)-\frac{1}{2 \pi i} \int_{\Gamma} \frac{\mu^{-\alpha}}{\mu-\lambda} R\left(\mu, \tilde{A}_{h}\right) \mathrm{d} \mu .
\end{aligned}
$$

On the other hand, similar to the proof of Proposition 3.4,

$$
\left|\int_{\Gamma} \frac{\mu^{-\alpha}}{\mu-\lambda} R\left(\mu, \tilde{A}_{h}\right) \mathrm{d} \mu\right| \leq c \int_{\Gamma} \frac{1}{|\mu|^{\alpha+1}}\left\|R\left(\mu, \tilde{A}_{h}\right)\right\| \mathrm{d} \mu \leq c^{\prime},
$$

where $c$ is independent of $h$. Therefore for all $\lambda \in S$, with $|\lambda|>N$, we have

$$
\left\|R\left(\lambda, \tilde{A}_{h}\right) \tilde{A}_{h}^{-\alpha}\right\| \leq \frac{c}{|\lambda|^{\alpha}}\left\|R\left(\lambda, \tilde{A}_{h}\right)\right\|+c \leq c \frac{1+|\lambda|^{\alpha}}{|\lambda|^{\alpha}}+c \leq c^{\prime \prime} .
$$

Now, for $\lambda \in S$ such that $|\lambda| \leq N$, we have

$$
\left\|R\left(\lambda, \tilde{A}_{h}\right) \tilde{A}_{h}^{-\alpha}\right\| \leq\left\|R\left(\lambda, \tilde{A}_{h}\right)\right\|\left\|\tilde{A}_{h}^{-\alpha}\right\| \leq c\left(1+|\lambda|^{\alpha}\right) \leq c\left(1+N^{\alpha}\right),
$$

which completes the proof with Proposition 3.4.

Lemma 3.6. If (3.1)-(3.5) are satisfied, then there exists $c>0$ independent of $h$ such that

$$
\sup _{\substack{h \in\left(0, h^{*}\right) \\ \operatorname{Re}>0}}\left\|R\left(\lambda, \tilde{A}_{h}\right) \tilde{A}_{h}^{-\alpha}\right\| \leq c .
$$

Proof. For all $h \in\left(0, h^{*}\right), m>0$, and $B>\max \{2 m, 1\}$, consider $F_{h}(\lambda)=R\left(\lambda, \tilde{A}_{h}\right) \lambda^{-\alpha}\left(1-\frac{\lambda^{2}}{B^{2}}\right)$ on the domain $D=\left\{\lambda \in \mathbb{C}: \operatorname{Re} \lambda>0, m<|\lambda| \leq \frac{B}{2}\right\} . F_{h}$, by the maximum principle, attains its maximum for $|\lambda|=\frac{B}{2}$. Therefore,

$$
\left|F_{h}(\lambda)\right| \leq \frac{c}{R e \lambda}
$$

If there exists $\epsilon>0$ such that $\operatorname{Re} \lambda>\epsilon$, then $\left|F_{h}(\lambda)\right| \leq c$.

Otherwise, for $0<R e \lambda<\epsilon$, using the resolvent identity

$$
R\left(\lambda, \tilde{A}_{h}\right)=R\left(i \operatorname{Im} \lambda, \tilde{A}_{h}\right)-\operatorname{Re} \lambda R\left(i \operatorname{Im} \lambda, \tilde{A}_{h}\right) R\left(\lambda, \tilde{A}_{h}\right)
$$

then, as $|\operatorname{Im} \lambda| \geq m-\epsilon$ for all $m>0$, we have

$$
\left\|R\left(\lambda, \tilde{A}_{h}\right)\right\| \leq c|\operatorname{Im} \lambda|^{\alpha} .
$$


Therefore,

$$
\left|F_{h}(\lambda)\right| \leq c|\operatorname{Im} \lambda|^{\alpha}|\lambda|^{-\alpha}\left|1-\frac{\lambda^{2}}{B^{2}}\right| \leq c .
$$

Hence, in all cases, there exists $c>0$ independent of $B$ such that

$$
\left|F_{h}(\lambda)\right| \leq c .
$$

As a result, for all $\lambda \in D$,

$$
\left\|R\left(\lambda, \tilde{A}_{h}\right)\right\| \leq \frac{c|\lambda|^{\alpha}}{\left|1-\frac{\lambda^{2}}{B^{2}}\right|} \leq c|\lambda|^{\alpha} \leq c\left(1+|\lambda|^{\alpha}\right) .
$$

If $0<\operatorname{Re} \lambda \leq|\lambda| \leq m$, then by (3.10) and Assumption (3.2), we get

$$
\left\|R\left(\lambda, \tilde{A}_{h}\right)\right\| \leq c\left\|R\left(i \operatorname{Im} \lambda, \tilde{A}_{h}\right)\right\| \leq c \leq c\left(1+|\lambda|^{\alpha}\right) .
$$

Letting $B \rightarrow+\infty$ yields

$$
\left\|R\left(\lambda, \tilde{A}_{h}\right)\right\| \leq c\left(1+|\lambda|^{\alpha}\right), \forall \operatorname{Re} \lambda>0 .
$$

Applying Lemma 3.5, we get for $0 \leq \operatorname{Re} \lambda \leq m$,

$$
\left\|R\left(\lambda, \tilde{A}_{h}\right) \tilde{A}_{h}^{-\alpha}\right\| \leq c .
$$

In addition, if $\operatorname{Re} \lambda \geq m$, by the Hille Yosida theorem and Proposition 3.4, there exists some positive constants $c_{1}$ and $c_{2}$ such that

$$
\left\|R\left(\lambda, \tilde{A}_{h}\right) \tilde{A}_{h}^{-\alpha}\right\| \leq c_{1} \frac{\left\|\tilde{A}_{h}^{-\alpha}\right\|}{\operatorname{Re} \lambda} \leq c_{2} .
$$

In all cases, we get (3.9).

The last lemma in this section gives the necessary and sufficient conditions for the boundedness of any family of $C_{0}$ semigroups $\left(S_{h}(t)\right) \underset{h \in\left(0,0, h^{*}\right)}{t}$.

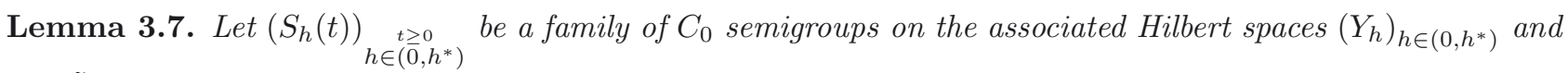

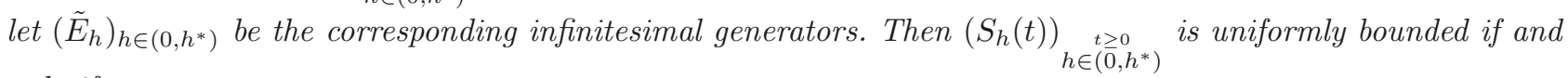
only if

(i) $\{\lambda \in \mathbb{C}: \operatorname{Re} \lambda>0\} \subseteq \rho\left(\tilde{E}_{h}\right), \forall h \in\left(0, h^{*}\right)$

(ii) There exists $c>0$ independent of $h$ such that

$$
\sup _{\substack{\xi>0 \\ h \in\left(0, h^{*}\right)}} \xi \int_{\mathbb{R}}\left(\left\|R\left(\xi+i \eta, \tilde{E}_{h}\right)\right\|^{2}+\left\|R\left(\xi+i \eta, \tilde{E}_{h}^{*}\right)\right\|^{2}\right) \mathrm{d} \eta \leq c .
$$

Proof. First, we assume that $\left(S_{h}(t)\right)$ is uniformly bounded. Then (i) holds by the Hille-Yosida theorem. As for (ii), we only need to prove that

$$
\sup _{\substack{\xi>0 \\ h \in\left(0, h^{*}\right)}} \xi \int_{\mathbb{R}}\left\|R\left(\xi+i \eta, \tilde{E}_{h}\right) x_{h}\right\|^{2} \mathrm{~d} \eta \leq c\left\|x_{h}\right\|^{2}, \forall x_{h} \in Y_{h}
$$

because according to the theory of adjoint semigroups, (see [30]), $S^{*}(t)$ is a $C_{0}$ semigroup with the same properties as $S(t)$. 
Similar to the proof of Theorem 1.1 in [21], we have for all $h \in\left(0, h^{*}\right), x_{h} \in Y_{h}$

$$
\left\|R\left(\xi+i \eta, \tilde{E}_{h}\right) x_{h}\right\|^{2}=\int_{\mathbb{R}} \mathrm{e}^{-i \eta s} f_{h}(s) \mathrm{d} s,
$$

where

$$
f_{h}(s)=\int_{\max \{0,-s\}}^{+\infty} \mathrm{e}^{-\xi(s+2 u)}<S_{h}(u+s) x_{h}, S_{h}(u) x_{h}>_{Y_{h}, Y_{h}} \mathrm{~d} u .
$$

For $s \geq 0$, since $\left(S_{h}(t)_{h \in\left(0, h^{*}\right)}\right.$ is uniformly bounded, i.e. $\sup _{h \in\left(0, h^{*}\right)}\left\|S_{h}(t)\right\| \leq M$, we have

$$
\left|f_{h}(s)\right| \leq \int_{0}^{+\infty} M^{2}\left\|x_{h}\right\|^{2} \mathrm{e}^{-\xi(s+2 u)} \mathrm{d} u=\frac{M^{2}\left\|x_{h}\right\|^{2}}{2 \xi} \mathrm{e}^{-\xi s} \leq \frac{M^{2}\left\|x_{h}\right\|^{2}}{2 \xi} .
$$

For $s<0$, we have

$$
\left|f_{h}(s)\right| \leq \int_{-s}^{+\infty} M^{2}\left\|x_{h}\right\|^{2} \mathrm{e}^{-\xi(s+2 u)} \mathrm{d} u=\frac{M^{2}\left\|x_{h}\right\|^{2} \mathrm{e}^{\xi s}}{2 \xi} \leq \frac{M^{2}\left\|x_{h}\right\|^{2}}{2 \xi}
$$

Hence, $f_{h} \in \mathcal{L}^{1}(\mathbb{R}) \cap \mathcal{L}^{\infty}(\mathbb{R})$ and

$$
\mathfrak{F}\left(f_{h}(s)\right)=\frac{1}{\sqrt{2 \pi}}\left\|R\left(\xi+i \eta, \tilde{E}_{h}\right) x_{h}\right\|^{2} .
$$

Using Lemma 21.50 in [20], it follows that

$$
\frac{1}{2 \pi} \int_{\mathbb{R}}\left\|R\left(\xi+i \eta, \tilde{E}_{h}\right) x_{h}\right\|^{2} \mathrm{~d} \eta=\frac{1}{\sqrt{2 \pi}} \int_{\mathbb{R}} \mathfrak{F}\left(f_{h}\right)(\tau) d \tau \leq c\left\|f_{h}\right\|_{L^{\infty}} \leq \frac{c M^{2}\left\|x_{h}\right\|^{2}}{2 \xi}
$$

Hence, (3.11) is verified.

As for the sufficient condition, since $\{\lambda \in \mathbb{C}: \operatorname{Re} \lambda>0\} \subseteq \rho\left(\tilde{E}_{h}\right)$, with $\sigma=\frac{1}{t}$, we get for all $x_{h} \in Y_{h}$

$$
\begin{aligned}
S_{h}(t) x_{h} & =\frac{1}{2 \pi i} \int_{\sigma-i \infty}^{\sigma+i \infty} \mathrm{e}^{\lambda t}\left(\lambda-\tilde{E}_{h}\right)^{-1} x_{h} \mathrm{~d} \lambda, \\
& =\frac{1}{2 \pi i} \int_{\sigma-i \infty}^{\sigma+i \infty} \frac{\mathrm{e}^{\lambda t}}{t}\left(\lambda-\tilde{E}_{h}\right)^{-2} x_{h} \mathrm{~d} \lambda+\left.\frac{\mathrm{e}^{\lambda t}}{t}\left(\lambda-\tilde{E}_{h}\right)^{-1} x_{h}\right|_{\sigma-i \infty} ^{\sigma+i \infty} .
\end{aligned}
$$

But $\left.\frac{\mathrm{e}^{\lambda t}}{t}\left(\lambda-\tilde{E}_{h}\right)^{-1} x_{h}\right|_{\sigma-i \infty} ^{\sigma+i \infty}=0$ since according to Lemma 2.1 of [33], under condition (ii), we have $\left\|R\left(\lambda, \tilde{E}_{h}\right) x_{h}\right\| \rightarrow 0$ as $|\lambda| \rightarrow+\infty$ whenever $\operatorname{Re} \lambda>0$. Therefore,

$$
\begin{aligned}
\left\langle S_{h}(t) x_{h}, y_{h}\right\rangle_{Y_{h}, Y_{h}} & =\left\langle\frac{1}{2 \pi i t} \int_{\sigma-i \infty}^{\sigma+i \infty} \mathrm{e}^{\lambda t}\left(\lambda-\tilde{E}_{h}\right)^{-2} x_{h} \mathrm{~d} \lambda, y_{h}\right\rangle_{Y_{h}, Y_{h}} \\
& =\frac{1}{2 \pi i t} \int_{\sigma-i \infty}^{\sigma+i \infty} \mathrm{e}^{\lambda t}\left\langle\left(\lambda-\tilde{E}_{h}\right)^{-2} x_{h}, y_{h}\right\rangle_{Y_{h}, Y_{h}} \mathrm{~d} \lambda .
\end{aligned}
$$

Let $\lambda=\frac{1}{t}+i \eta$ with $\eta \in \mathbb{R}$. Then

$$
\left\langle S_{h}(t) x_{h}, y_{h}\right\rangle_{Y_{h}, Y_{h}}=\frac{e}{2 \pi t} \int_{\mathbb{R}} \mathrm{e}^{i \eta t}\left\langle R^{2}\left(\frac{1}{t}+i \eta, \tilde{E}_{h}\right) x_{h}, y_{h}\right\rangle_{Y_{h}, Y_{h}} \mathrm{~d} \eta .
$$


Hölder's inequality yields

$$
\begin{aligned}
\left|\left\langle S_{h}(t) x_{h}, y_{h}\right\rangle_{Y_{h}, Y_{h}}\right| & =\left|\frac{e}{2 \pi t} \int_{\mathbb{R}} \mathrm{e}^{i \eta t}\left\langle R\left(\frac{1}{t}+i \eta, \tilde{E}_{h}\right) x_{h}, R\left(\frac{1}{t}+i \eta, \tilde{E}_{h}^{*}\right) y_{h}\right\rangle_{Y_{h}, Y_{h}} \mathrm{~d} \eta\right| \\
& \leq \frac{e}{2 \pi t}\left(\int_{\mathbb{R}}\left\|R\left(\frac{1}{t}+i \eta, \tilde{E}_{h}\right) x_{h}\right\|^{2} \mathrm{~d} \eta\right)^{\frac{1}{2}}\left(\int_{\mathbb{R}}\left\|R\left(\frac{1}{t}+i \eta, \tilde{E}_{h}^{*}\right) y_{h}\right\|^{2} \mathrm{~d} \eta\right)^{\frac{1}{2}} \\
& \leq c\left\|x_{h}\right\|\left\|y_{h}\right\| .
\end{aligned}
$$

Therefore

$$
\left\|S_{h}(t)\right\| \leq c, \forall h \in\left(0, h^{*}\right) .
$$

Now, we display the main theorem which leads to the polynomial stability of the discretized problem (1.14).

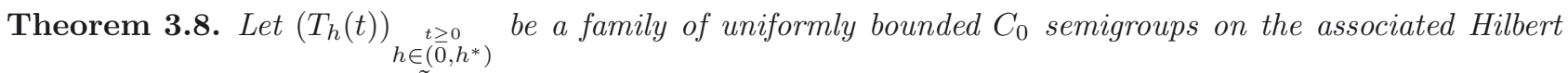
spaces $\left(X_{h}\right)_{h \in\left(0, h^{*}\right)}$ and let $\left(\tilde{A}_{h}\right)_{h \in\left(0, h^{*}\right)}$ be the corresponding infinitesimal generators such that (3.1) and (3.2) are satisfied. Then for a fixed $\alpha>0$, the following statements are equivalent:

$$
\sup _{h \in\left(0, h^{*}\right)}\left\|R\left(i s, \tilde{A}_{h}\right)\right\|=O\left(|s|^{\alpha}\right), \quad|s| \rightarrow \infty
$$

$$
\sup _{h \in\left(0, h^{*}\right)}\left\|T_{h}(t) \tilde{A}_{h}^{-\alpha}\right\|=O\left(t^{-1}\right), \quad t \rightarrow+\infty
$$

$$
\sup _{h \in\left(0, h^{*}\right)}\left\|T_{h}(t) \tilde{A}_{h}^{-1}\right\|=O\left(t^{\frac{-1}{\alpha}}\right), \quad t \rightarrow+\infty .
$$

Proof. We begin to prove (ii) $\Leftrightarrow$ (iii). We adapt the proof found in [7] Proposition 3.1 without the discretization parameter $h$. Given (ii), we have

$$
\left\|T_{h}(t) \tilde{A}_{h}^{-\alpha n}\right\|=\left\|\left[T_{h}\left(\frac{t}{n}\right) \tilde{A}_{h}^{-\alpha}\right]^{n}\right\| \leq c\left(\frac{n}{t}\right)^{n} \leq c(n) t^{-n}, \forall n \in \mathbb{N}^{*}, h \in\left(0, h^{*}\right), t \rightarrow+\infty .
$$

According to the moment inequality in Theorem II.5.34 of [13], we remark that there exists a positive constant $L$ independent of h such that, for all $\nu \in(0,1)$, we have

$$
\begin{aligned}
\left\|T_{h}(t) \tilde{A}_{h}^{-\alpha n \nu}\right\| & =\left\|\tilde{A}_{h}^{\alpha n(1-\nu)} T_{h}(t) \tilde{A}_{h}^{-\alpha n}\right\| \\
& \leq L\left\|\tilde{A}_{h}^{\alpha n} T_{h}(t) \tilde{A}_{h}^{-\alpha n}\right\|^{(1-\nu)}\left\|T_{h}(t) \tilde{A}_{h}^{-\alpha n}\right\|^{\nu} \\
& \leq L M^{1-\nu} c^{\nu}(n) t^{-n \nu} .
\end{aligned}
$$

Choose $\nu=\frac{1}{\alpha n}$ with $n>\frac{1}{\alpha}$ to get

$$
\left\|T_{h}(t) \tilde{A}_{h}^{-1}\right\| \leq c(n) t^{-\frac{1}{\alpha}}
$$

Conversely, assume that (iii) holds. Then

$$
\left\|T_{h}(t) \tilde{A}_{h}^{-n}\right\|=\left\|\left[T_{h}\left(\frac{t}{n}\right) \tilde{A}_{h}^{-1}\right]^{n}\right\| \leq\left(\frac{t}{n}\right)^{\frac{-n}{\alpha}} \leq n^{\frac{n}{\alpha}} t^{-\frac{n}{\alpha}}, \forall n \in \mathbb{N}^{*} .
$$


Therefore,

$$
\begin{aligned}
\left\|T_{h}(t) \tilde{A}_{h}^{-n \nu}\right\| & \leq c\left\|\tilde{A}_{h}^{n} T_{h}(t) \tilde{A}_{h}^{-n}\right\|^{1-\nu}\left\|T_{h}(t) \tilde{A}_{h}^{-n}\right\|^{\nu} \\
& \leq c M^{1-\nu} c(n)^{\nu} t^{-\frac{n \nu}{\alpha}}, \quad \forall \nu \in(0,1) .
\end{aligned}
$$

Take $\nu=\frac{\alpha}{n}$ with $n>\alpha$ to get

$$
\sup _{h \in\left(0, h^{*}\right)}\left\|T_{h}(t) \tilde{A}_{h}^{-\alpha}\right\|=O\left(t^{-1}\right) .
$$

Now, we prove the implication $(i i) \Rightarrow(i)$ (for the continuous case, see [8]). Given (ii), define

$$
m_{1}(t)=\sup _{\substack{h \in\left(0, h^{*}\right) \\ s \geq t}}\left\|T_{h}(s) \tilde{A}_{h}^{-1}\right\| .
$$

Notice that $m_{1}(t)$ is non increasing. Let $u_{0 h} \in \mathcal{D}\left(\tilde{A}_{h}\right), f_{0 h}=\left(-\tilde{A}_{h}+i \tau\right) u_{0 h}, \tau \in \mathbb{R}$, and let $v_{h}(t)=\mathrm{e}^{i t \tau} u_{0 h}$. We have

$$
\left\{\begin{aligned}
\partial_{t} v_{h}-\tilde{A}_{h} v_{h} & =i \tau \mathrm{e}^{i t \tau} u_{0 h}-\tilde{A}_{h}\left(\mathrm{e}^{i t \tau} u_{0 h}\right)=\mathrm{e}^{i t \tau} f_{0 h} \\
v_{h}(0) & =u_{0 h}
\end{aligned}\right.
$$

By the Duhamel formula,

$$
v_{h}=\mathrm{e}^{t \tilde{A}_{h}} u_{0 h}+\int_{0}^{t} \mathrm{e}^{(t-s) \tilde{A}_{h}} \mathrm{e}^{i \tau s} f_{0 h} \mathrm{~d} s .
$$

By the boundedness of the semigroup $\left(T_{h}(t)\right)$ and the definition of $m_{1}$, we have

$$
\begin{aligned}
\left\|u_{0 h}\right\|=\left\|v_{h}(t)\right\| & \leq\left\|T_{h}(t) \tilde{A}_{h}^{-1} \tilde{A}_{h} u_{0 h}\right\|+c t\left\|f_{0 h}\right\| \\
& \leq m_{1}(t)\left\|\tilde{A}_{h} u_{0 h}\right\|+c t\left\|f_{0 h}\right\| \\
& \leq m_{1}(t)\left(\left\|f_{0 h}\right\|+|\tau|\left\|u_{0 h}\right\|\right)+c t\left\|f_{0 h}\right\| .
\end{aligned}
$$

Apply the above inequality with $t=G(|\tau|)$ where

$$
G(\xi)=\left\{\begin{array}{ccc}
m_{1 r}^{-1}\left(\frac{1}{2(\xi+1)}\right) & \text { if } & \xi>0 \text { and } \frac{1}{2(\xi+1)} \leq m_{1}(0) \\
0 & \text { if } & \xi>0 \text { and } \frac{1}{2(\xi+1)}>m_{1}(0)
\end{array}\right.
$$

where $m_{1 r}^{-1}$ is the right inverse of $m_{1}$. Therefore,

$$
m_{1}(t)|\tau|=m_{1}(G(|\tau|))|\tau| \leq \frac{|\tau|}{2(|\tau|+1)} \leq \frac{1}{2} .
$$

Hence,

$$
\begin{aligned}
\frac{1}{2}\left\|u_{0 h}\right\| & \leq m_{1}(G(|\tau|))\left\|f_{0 h}\right\|+c G(|\tau|)\left\|f_{0 h}\right\| \\
& \leq \frac{\left\|f_{0 h}\right\|}{2(|\tau|+1)}+c G(|\tau|)\left\|f_{0 h}\right\| \\
& \leq\left(\frac{1}{2}+c G(|\tau|)\right)\left\|f_{0 h}\right\| .
\end{aligned}
$$

Consequently,

$$
\left\|\left(i \tau-\tilde{A}_{h}\right)^{-1}\right\| \leq 1+2 c G(|\tau|),
$$

i.e.,

$$
\sup _{h \in\left(0, h^{*}\right)}\left\|\left(i \tau-\tilde{A}_{h}\right)^{-1}\right\| \leq 1+2 c G(|\tau|) .
$$


Since, by $(i i i)$,

$$
\sup _{h \in\left(0, h^{*}\right)}\left\|T_{h}(t) \tilde{A}_{h}^{-1}\right\| \leq M t^{\frac{-1}{\alpha}}, \quad t \rightarrow+\infty,
$$

then, as $m_{1}$ is non-increasing, we get

$$
m_{1}(t) \leq M t^{\frac{-1}{\alpha}}, \quad t \rightarrow+\infty .
$$

Besides, as the inverse of $t^{\frac{-1}{\alpha}}$ is $t^{-\alpha}$, then

$$
G(\xi) \leq m_{1 r}^{-1}\left(\frac{1}{2(\xi+1)}\right) \leq C\left(\frac{1}{2(\xi+1)}\right)^{-\alpha}=C(2(\xi+1))^{\alpha} \leq c \xi^{\alpha}, \quad \xi \rightarrow+\infty .
$$

Finally, we get

$$
\sup _{h \in\left(0, h^{*}\right)}\left\|\left(i \tau-\tilde{A}_{h}\right)^{-1}\right\| \leq 1+2 c|\tau|^{\alpha} \leq c|\tau|^{\alpha}, \quad|\tau| \rightarrow+\infty .
$$

It remains to prove that $(i) \Rightarrow(i i)$. For this aim, for all $h \in\left(0, h^{*}\right)$, let $\mathbf{X}_{\mathbf{h}}=X_{h} \times X_{h}$ and consider the operator $\tilde{\mathbf{A}}_{\mathbf{h}}$ given by the operator matrix

$$
\tilde{\mathbf{A}}_{\mathbf{h}}=\left(\begin{array}{cc}
\tilde{A}_{h} & \tilde{A}_{h}^{-\alpha} \\
0 & \tilde{A}_{h}
\end{array}\right),
$$

where $\mathcal{D}\left(\tilde{\mathbf{A}}_{\mathbf{h}}\right)=\mathcal{D}\left(\tilde{A}_{h}\right) \times \mathcal{D}\left(\tilde{A}_{h}\right)$. For all $h \in\left(0, h^{*}\right)$ and all $\lambda_{h} \in \rho\left(\tilde{A}_{h}\right)$, we have

$$
R\left(\lambda_{h}, \tilde{\mathbf{A}}_{\mathbf{h}}\right)=\left(\begin{array}{cc}
R\left(\lambda_{h}, \tilde{A}_{h}\right) & R^{2}\left(\lambda_{h}, \tilde{A}_{h}\right) \tilde{A}_{h}^{-\alpha} \\
0 & R\left(\lambda_{h}, \tilde{A}_{h}\right)
\end{array}\right) .
$$

Indeed,

$$
R\left(\lambda_{h}, \tilde{\mathbf{A}}_{\mathbf{h}}\right)\left(\lambda_{h}-\tilde{\mathbf{A}}_{\mathbf{h}}\right)=\left(\lambda_{h}-\tilde{\mathbf{A}}_{\mathbf{h}}\right) R\left(\lambda_{h}, \tilde{\mathbf{A}}_{\mathbf{h}}\right)=\left(\begin{array}{cc}
I_{h} & 0 \\
0 & I_{h}
\end{array}\right) .
$$

Therefore, $\rho\left(\tilde{\mathbf{A}}_{\mathbf{h}}\right)=\rho\left(\tilde{A}_{h}\right)$ and for all $h \in\left(0, h^{*}\right)$, the operator $\tilde{\mathbf{A}}_{\mathbf{h}}$ is the generator of the $C_{0}$ semigroup $\left(\mathbf{T}_{\mathbf{h}}(t)\right)_{t \geq 0}$ on $\mathbf{X}_{\mathbf{h}}$ defined by

$$
\mathbf{T}_{\mathbf{h}}(t)=\left(\begin{array}{cc}
T_{h}(t) & t T_{h}(t) \tilde{A}_{h}^{-\alpha} \\
0 & T_{h}(t)
\end{array}\right)
$$

In fact,

$$
\begin{aligned}
\widehat{\mathbf{T}_{\mathbf{h}}(t)} & =\left(\begin{array}{cc}
\widehat{T_{h}(t)} & \widehat{t T_{h}(t)} \tilde{A}_{h}^{-\alpha} \\
0 & \widehat{T_{h}(t)}
\end{array}\right) \\
& =\left(\begin{array}{cc}
R\left(\lambda_{h}, \tilde{A}_{h}\right) R^{2}\left(\lambda_{h}, \tilde{A}_{h}\right) \tilde{A}_{h}^{-\alpha} \\
0 & R\left(\lambda_{h}, \tilde{A}_{h}\right)
\end{array}\right) \\
& =R\left(\lambda_{h}, \tilde{\mathbf{A}}_{\mathbf{h}}\right),
\end{aligned}
$$

where $\widehat{\mathbf{T}_{\mathbf{h}}(t)}$ is the Laplace transform of $T_{h}(t)$. Since for all $h \in\left(0, h^{*}\right)$ we have

$$
\left\|R\left(i s, \tilde{A}_{h}\right)\right\|=O\left(|s|^{\alpha}\right), \text { as }|s| \rightarrow+\infty,
$$

then by Lemma 3.6 we get

$$
\sup _{\substack{h \in\left(0, h^{*}\right) \\ \operatorname{Re\lambda }>0}}\left\|R\left(\lambda, \tilde{A}_{h}\right) \tilde{A}_{h}^{-\alpha}\right\| \leq c .
$$


Hence, for all $x_{h}=\left(x_{1 h}, x_{2 h}\right) \in \mathbf{X}_{\mathbf{h}}$, and $\operatorname{Re} \lambda_{h}>0$, we have

$$
\begin{aligned}
\left\|R\left(\lambda_{h}, \tilde{\mathbf{A}}_{\mathbf{h}}\right) x_{h}\right\|^{2} & =\left\|\left(\begin{array}{c}
R\left(\lambda_{h}, \tilde{A}_{h}\right) x_{1 h}+R^{2}\left(\lambda_{h}, \tilde{A}_{h}\right) \tilde{A}_{h}^{-\alpha} x_{2 h} \\
R\left(\lambda_{h}, \tilde{A}_{h}\right) x_{2 h}
\end{array}\right)\right\|^{2} \\
& \leq c\left(\left\|R\left(\lambda_{h}, \tilde{A}_{h}\right) x_{1 h}\right\|^{2}+\left\|R\left(\lambda_{h}, \tilde{A}_{h}\right) x_{2 h}\right\|^{2}\right) .
\end{aligned}
$$

Similarly, we have

$$
\left\|R\left(\lambda_{h}, \tilde{\mathbf{A}}_{\mathbf{h}}^{*}\right) x_{h}\right\|^{2} \leq c\left(\left\|R\left(\lambda_{h}, \tilde{A}_{h}^{*}\right) x_{1 h}\right\|^{2}+\left\|R\left(\lambda_{h}, \tilde{A}_{h}^{*}\right) x_{2 h}\right\|^{2}\right) .
$$

Indeed, we have

$$
\tilde{\mathbf{A}}_{\mathbf{h}}^{*}=\left(\begin{array}{cc}
\tilde{A}_{h}^{*}\left(\tilde{A}_{h}^{*}\right)^{-\alpha} \\
0 & \tilde{A}_{h}^{*}
\end{array}\right)
$$

In order to get

$$
\sup _{\substack{h \in\left(0, h^{*}\right) \\ \operatorname{Re\lambda }>0}}\left\|R\left(\lambda, \tilde{A}_{h}^{*}\right)\left(\tilde{A}_{h}^{*}\right)^{-\alpha}\right\| \leq c
$$

we must have at least

$$
\left\|R\left(i s, \tilde{A}_{h}^{*}\right)\right\|=O\left(|s|^{\alpha}\right), \text { as }|s| \rightarrow+\infty
$$

Actually, we have

$$
R\left(i s, \tilde{A}_{h}^{*}\right)=\left[\left(i s-\tilde{A}_{h}^{*}\right)\right]^{-1}=\left[\left(i s-\tilde{A}_{h}\right)^{*}\right]^{-1}=R\left(i s, \tilde{A}_{h}\right)^{*}
$$

Therefore, we get

$$
\left\|R\left(i s, \tilde{A}_{h}^{*}\right)\right\| \leq\left\|R\left(i s, \tilde{A}_{h}\right)\right\|=O\left(|s|^{\alpha}\right), \text { as }|s| \rightarrow+\infty .
$$

Now, by Lemma 3.7 , since for all $h \in\left(0, h^{*}\right), T_{h}(t)$ is a uniformly bounded family of $C_{0}$ semigroups, we get

$$
\sup _{\substack{\xi>0 \\ h \in\left(0, h^{*}\right)}} \xi \int_{\mathbb{R}}\left(\left\|R\left(\xi+i \eta, \tilde{A}_{h}\right) x_{h}\right\|^{2}\right)+\left(\left\|R\left(\xi+i \eta, \tilde{A}_{h}^{*}\right) x_{h}\right\|^{2}\right) \mathrm{d} \eta<\infty, \quad \forall x_{h} \in X_{h} .
$$

Hence,

$$
\sup _{\substack{\xi>0 \\ h \in\left(0, h^{*}\right)}} \xi \int_{\mathbb{R}}\left(\left\|R\left(\xi+i \eta, \tilde{\mathbf{A}}_{\mathbf{h}}\right) x_{h}\right\|^{2}\right)+\left(\left\|R\left(\xi+i \eta, \tilde{\mathbf{A}}_{\mathbf{h}}^{*}\right) x_{h}\right\|^{2}\right) \mathrm{d} \eta<\infty, \quad \forall x_{h} \in \mathbf{X}_{\mathbf{h}} .
$$

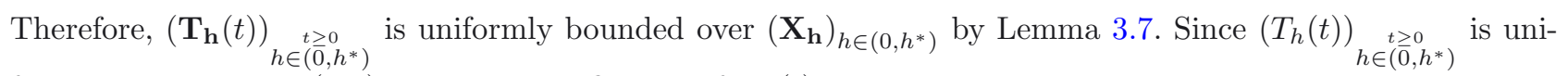
formly bounded over $\left(X_{h}\right)_{h \in\left(0, h^{*}\right)}$, the definition of $\mathbf{T}_{\mathbf{h}}(t)$ implies that

$$
\sup _{\substack{t \geq 0 \\ h \in\left(0, h^{*}\right)}}\left\|t T_{h}(t) \tilde{A}_{h}^{-\alpha}\right\|<+\infty
$$

\section{Preliminary lemmas}

In this section, we fix $l \in \mathbb{N}, l$ even. We introduce the Hilbert space $X_{h}=V_{h} \times V_{h}$ and the operator $\tilde{A}_{l, h}: X_{h} \rightarrow X_{h}$ defined by

$$
\tilde{A}_{l, h}=\left(\begin{array}{cc}
0 & \left(1+h^{\theta}\right)^{-1}\left(I+h^{\theta} A_{h}^{\frac{l}{2}}\right) \\
-\left(1+h^{\theta}\right)^{-1}\left(I+h^{\theta} A_{h}^{\frac{l}{2}}\right) A_{h} & -h^{\theta} A_{h}^{1+\frac{l}{2}}-B_{h} B_{h}^{*}
\end{array}\right) .
$$


The space $X_{h}$ is here equipped with the inner product

$$
\left(\left(\begin{array}{c}
u_{h} \\
v_{h}
\end{array}\right),\left(\begin{array}{c}
\tilde{u}_{h} \\
\tilde{v}_{h}
\end{array}\right)\right)_{X_{h}}=a\left(u_{h}, \tilde{u}_{h}\right)+\left(v_{h}, \tilde{v}_{h}\right), \quad \forall\left(u_{h}, v_{h}\right),\left(\tilde{u}_{h}, \tilde{v}_{h}\right) \in X_{h}
$$

with associated norm $\|\cdot\|_{X_{h}}$. Therefore, the system (1.14) is equivalent to the following first order system in $X_{h}$ :

$$
\dot{z}_{h}(t)=\tilde{A}_{l, h} z_{h}(t), \quad z_{h}(0)=z_{0 h}
$$

where $z_{h}(t)=\left(\begin{array}{c}\omega_{h}(t) \\ \left(1+h^{\theta}\right)\left(I+h^{\theta} A_{h}^{\frac{l}{2}}\right)^{-1} \dot{\omega}_{h}(t)\end{array}\right)$ and $z_{0 h}=\left(\begin{array}{c}\omega_{0 h} \\ \omega_{1 h}\end{array}\right)$. Note that we recover the system (1.9) in the particular case $l=0$. We define the sesquilinear form $a^{l}(.,$.$) on V_{h}$ by

$$
a^{l}\left(u_{h}, v_{h}\right)=\left(A_{h}^{1+\frac{l}{2}} u_{h}, v_{h}\right), \forall\left(u_{h}, v_{h}\right) \in V_{h} \times V_{h},
$$

i.e.

$$
a^{l}\left(u_{h}, v_{h}\right)=\sum_{k=1}^{N(h)} c_{k} \overline{d_{k}} \lambda_{k, h}^{2+l}
$$

for $u_{h}=\sum_{k=1}^{N(h)} c_{k} \varphi_{k, h}$ and $v_{h}=\sum_{k=1}^{N(h)} d_{k} \varphi_{k, h}$. Remark that $a^{0}(.,)=.a(.,$.$) defined in (1.3).$

We easily prove that $\tilde{A}_{l, h}$ is maximal dissipative in $X_{h}$, hence $\left(T_{l, h}(t)\right)=\left(\mathrm{e}^{t \tilde{A}_{l, h}}\right)$ forms a family of $C_{0}$ semigroups of contractions in $X_{h}$. In the sequel we prove that the family $\left(\tilde{A}_{l, h}\right)_{h \in\left(0, h^{*}\right)}$ satisfies condition i) in Theorem 3.1 and the properties (3.1) and (3.2) of Subsection 3.2. Condition i) in Theorem 3.1 or (3.1) in Section 3.2 is satisfied due to the following lemma:

Lemma 4.1. The spectrum of the operator $\tilde{A}_{l, h}$ contains no point on the imaginary axis.

Proof. Suppose that $\left(\begin{array}{l}\varphi_{h} \\ \psi_{h}\end{array}\right) \in X_{h}$ and $\omega \in \mathbb{R}$ are such that

$$
\tilde{A}_{l, h}\left(\begin{array}{c}
\varphi_{h} \\
\psi_{h}
\end{array}\right)=i \omega\left(\begin{array}{c}
\varphi_{h} \\
\psi_{h}
\end{array}\right)
$$

Then, by using the definition (4.1) of $\tilde{A}_{l, h}$, we have

$$
\left\{\begin{array}{c}
\psi_{h}=i \omega\left(1+h^{\theta}\right)\left(I+h^{\theta} A_{h}^{\frac{l}{2}}\right)^{-1} \varphi_{h} \\
-\left(1+h^{\theta}\right)^{-1}\left(I+h^{\theta} A_{h}^{\frac{l}{2}}\right) A_{h} \varphi_{h}-i \omega\left(1+h^{\theta}\right)\left(h^{\theta} A_{h}^{1+\frac{l}{2}}+B_{h} B_{h}^{*}\right)\left(I+h^{\theta} A_{h}^{\frac{l}{2}}\right)^{-1} \varphi_{h}= \\
-\omega^{2}\left(1+h^{\theta}\right)\left(I+h^{\theta} A_{h}^{\frac{l}{2}}\right)^{-1} \varphi_{h} .
\end{array}\right.
$$

Let $\chi_{h}=\left(1+h^{\theta}\right)\left(I+h^{\theta} A_{h}^{\frac{l}{2}}\right)^{-1} \varphi_{h}$ then the second relation of (4.3) becomes

$$
\left(1+h^{\theta}\right)^{-2}\left(I+h^{\theta} A_{h}^{\frac{l}{2}}\right)^{2} A_{h} \chi_{h}+i \omega\left(h^{\theta} A_{h}^{1+\frac{l}{2}}+B_{h} B_{h}^{*}\right) \chi_{h}=\omega^{2} \chi_{h} .
$$

If $\omega=0$, then taking the inner product of (4.4) with $\chi_{h} \in V_{h}$, we get $\left(I+h^{\theta} A_{h}^{\frac{l}{2}}\right) A_{h}^{\frac{1}{2}} \chi_{h}=0$ and hence $\chi_{h}=0$ which implies by the definition of $\chi_{h}$ that $\varphi_{h}=\psi_{h}=0$. 
It then remains to consider the case $\omega \neq 0$. In that case, we take the imaginary part of the inner product (in $H$ ) of (4.4) with $\chi_{h} \in V_{h}$ to obtain

$$
\begin{aligned}
0 & =\omega h^{\theta}\left(A_{h}^{1+\frac{l}{2}} \chi_{h}, \chi_{h}\right)+\omega\left(B_{h} B_{h}^{*} \chi_{h}, \chi_{h}\right) \\
& =\omega h^{\theta}\left(A_{h}^{\frac{1}{2}+\frac{l}{4}} \chi_{h}, A_{h}^{\frac{1}{2}+\frac{l}{4}} \chi_{h}\right)+\omega\left(B_{h}^{*} \chi_{h}, B_{h}^{*} \chi_{h}\right)_{U},
\end{aligned}
$$

that is to say

$$
h^{\theta}\left\|A_{h}^{\frac{1}{2}+\frac{l}{4}} \chi_{h}\right\|^{2}+\left\|B_{h}^{*} \chi_{h}\right\|_{U}^{2}=0 .
$$

This leads to $\chi_{h}=0$, and hence $\varphi_{h}=\psi_{h}=0$.

Our main goal is to prove condition (ii) of Theorem 3.1 in the case $l=0$ and condition (i) of Theorem 3.8 as well as (3.2) in the case $l \geq 2$ and $\alpha=2 l$. In that last case $(l \geq 2)$, these two conditions are equivalent to

$$
\sup _{h \in\left(0, h^{*}\right), s \in \mathbb{R}}\left(1+|s|^{2 l}\right)^{-1}\left\|R\left(i s, \tilde{A}_{l, h}\right)\right\|_{\mathcal{L}\left(X_{h}\right)}<\infty .
$$

To prove this above property, we use a contradiction argument. More precisely, we will assume that, for all $n \in \mathbb{N}$, there exist $h_{n} \in\left(0, h^{*}\right), \omega_{n} \in \mathbb{R}$ and $z_{n}=\left(\begin{array}{c}\varphi_{n} \\ \psi_{n}\end{array}\right) \in X_{h_{n}}$ such that

$$
\left\|z_{n}\right\|_{X_{h_{n}}}^{2}=a\left(\varphi_{n}, \varphi_{n}\right)+\left\|\psi_{n}\right\|^{2}=1, \forall n \in \mathbb{N}
$$

and

$$
\left(1+\left|\omega_{n}\right|^{2 l}\right)\left\|i \omega_{n} z_{n}-\tilde{A}_{l, h_{n}} z_{n}\right\|_{X_{h_{n}}} \rightarrow 0, \text { as } n \rightarrow \infty,
$$

where $l=0$ in the setting of Theorem 3.1.

Lemma 4.2. Assume that the sequences $\left(h_{n}\right),\left(\omega_{n}\right),\left(z_{n}\right)$ satisfy (4.6) and (4.7). Then, we have

$$
\left(1+\left|\omega_{n}\right|^{2 l}\right)\left(h_{n}^{\theta} a^{l}\left(\psi_{n}, \psi_{n}\right)+\left\|B_{h_{n}}^{*} \psi_{n}\right\|_{U}^{2}\right) \rightarrow 0 \text {, as } n \rightarrow \infty
$$

and

$$
\lim _{n \rightarrow \infty} a\left(\varphi_{n}, \varphi_{n}\right)=\lim _{n \rightarrow \infty}\left\|\psi_{n}\right\|^{2}=\frac{1}{2} .
$$

Proof. For (4.8), we take the inner product in $X_{h_{n}}$ of $i \omega_{n} z_{n}-\tilde{A}_{l, h_{n}} z_{n}$ with $z_{n}$ and take the real part. We obtain

$$
\begin{aligned}
& \Re\left(i \omega_{n} z_{n}-\tilde{A}_{l, h_{n}} z_{n}, z_{n}\right)_{X_{h_{n}}} \\
= & -\Re\left(\left(\begin{array}{c}
\left(1+h_{n}^{\theta}\right)^{-1}\left(I+h_{n}^{\theta} A_{h_{n}}^{\frac{l}{2}}\right) \psi_{n} \\
-\left(1+h_{n}^{\theta}\right)^{-1}\left(I+h_{n}^{\theta} A_{h_{n}}^{\frac{l}{2}}\right) A_{h_{n}} \varphi_{n}-h_{n}^{\theta} A_{h_{n}}^{1+\frac{l}{2}} \psi_{n}-B_{h_{n}} B_{h_{n}}^{*} \psi_{n}
\end{array}\right),\left(\begin{array}{c}
\varphi_{n} \\
\psi_{n}
\end{array}\right)\right) \\
= & \Re\left(-\left(1+h_{n}^{\theta}\right)^{-1}\left(\left(I+h_{n}^{\theta} A_{h}^{\frac{l}{2}}\right) A_{h_{n}} \psi_{n}, \varphi_{n}\right)+\left(1+h_{n}^{\theta}\right)^{-1}\left(\left(I+h_{n}^{\theta} A_{h_{n}}^{\frac{l}{2}}\right) A_{h_{n}} \varphi_{n}, \psi_{n}\right)\right) \\
& \left.+\left(h_{n}^{\theta} A_{h_{n}}^{1+\frac{l}{2}} \psi_{n}+B_{h_{n}} B_{h_{n}}^{*} \psi_{n}, \psi_{n}\right)\right) \\
= & \left(h_{n}^{\theta} A_{h_{n}}^{1+\frac{l}{2}} \psi_{n}+B_{h_{n}} B_{h_{n}}^{*} \psi_{n}, \psi_{n}\right) .
\end{aligned}
$$

Then

$$
\left(1+\left|\omega_{n}\right|^{2 l}\right) \Re\left(i \omega_{n} z_{n}-\tilde{A}_{l, h_{n}} z_{n}, z_{n}\right)_{X_{h_{n}}}=\left(1+\left|\omega_{n}\right|^{2 l}\right)\left(h_{n}^{\theta} a^{l}\left(\psi_{n}, \psi_{n}\right)+\left\|B_{h_{n}}^{*} \psi_{n}\right\|_{U}^{2}\right) \rightarrow 0 \text { by }(4.7) .
$$


In order to prove (4.9), we introduce the operator

$$
A_{1 h_{n}}=\left(1+h_{n}^{\theta}\right)^{-1}\left(I+h_{n}^{\theta} A_{h_{n}}^{\frac{l}{2}}\right)\left(\begin{array}{cc}
0 & I \\
-A_{h_{n}} & 0
\end{array}\right) .
$$

We have

$$
\tilde{A}_{l, h_{n}}\left(\begin{array}{c}
\varphi_{n} \\
\psi_{n}
\end{array}\right)=A_{1 h_{n}}\left(\begin{array}{c}
\varphi_{n} \\
\psi_{n}
\end{array}\right)-\left(\begin{array}{c}
0 \\
h_{n}^{\theta} A_{h_{n}}^{1+\frac{l}{2}} \psi_{n}+B_{h_{n}} B_{h_{n}}^{*} \psi_{n}
\end{array}\right), \forall\left(\begin{array}{l}
\varphi_{n} \\
\psi_{n}
\end{array}\right) \in X_{h_{n}}
$$

We take the norm $\|\cdot\|_{X_{h_{n}}}$ of $i \omega_{n} z_{n}-A_{1 h_{n}} z_{n}+\left(\begin{array}{c}0 \\ h_{n}^{\theta} A_{h_{n}}^{1+\frac{l}{2}} \psi_{n}\end{array}\right)$ to obtain

$$
\begin{aligned}
& \left(1+\left|\omega_{n}\right|^{2 l}\right)\left\|i \omega_{n} z_{n}-A_{1 h_{n}} z_{n}+\left(\begin{array}{c}
0 \\
h_{n}^{\theta} A_{h_{n}}^{1+\frac{l}{2}} \psi_{n}
\end{array}\right)\right\|_{X_{h_{n}}}^{2} \\
= & \left.\left(1+\left|\omega_{n}\right|^{2 l}\right)\left\|i \omega_{n} z_{n}-\tilde{A}_{l, h_{n}} z_{n}-\left(\begin{array}{c}
0 \\
B_{h_{n}} B_{h_{n}}^{*} \psi_{n}
\end{array}\right)\right\|_{X_{h_{n}}}^{2} \|^{2}\right) \\
\leq & 2\left(1+\left|\omega_{n}\right|^{2 l}\right)\left(\left\|i \omega_{n} z_{n}-\tilde{A}_{l, h_{n}} z_{n}\right\|_{X_{h_{n}}}^{2}+\left\|B_{h_{n}} B_{h_{n}}^{*} \psi_{n}\right\|^{2}\right) \\
\leq & C\left(1+\left|\omega_{n}\right|^{2 l}\right)\left(\left\|i \omega_{n} z_{n}-\tilde{A}_{l, h_{n}} z_{n}\right\|_{X_{h_{n}}}^{2}+\left\|B_{h_{n}}^{*} \psi_{n}\right\|_{U}^{2}\right) \rightarrow 0,
\end{aligned}
$$

by (4.7) and (4.8). Therefore

$$
\left(1+\left|\omega_{n}\right|^{2 l}\right)\left\|i \omega_{n} z_{n}-A_{1 h_{n}} z_{n}+\left(\begin{array}{c}
0 \\
h_{n}^{\theta} A_{h_{n}}^{1+\frac{l}{2}} \psi_{n}
\end{array}\right)\right\|_{X_{h_{n}}}^{2} \rightarrow 0 .
$$

We can now prove (4.9). If $l=0$, then by Lemma 4.3 below there exists $n_{0} \in \mathbb{N}$ such that the sequence $\left(\left|\omega_{n}\right|\right)_{n \geq n_{0}}$ is bounded away from zero. Hence, we may write

$$
\begin{aligned}
\Im\left(i \omega_{n} z_{n}-A_{1 h_{n}} z_{n}+\left(\begin{array}{c}
0 \\
h_{n}^{\theta} A_{h_{n}}^{1+\frac{l}{2}} \psi_{n}
\end{array}\right), \frac{1}{\omega_{n}}\left(\begin{array}{c}
\varphi_{n} \\
-\psi_{n}
\end{array}\right)\right)_{X_{h_{n}}} & =\left(\left(\begin{array}{c}
\varphi_{n} \\
\psi_{n}
\end{array}\right),\left(\begin{array}{c}
\varphi_{n} \\
-\psi_{n}
\end{array}\right)\right)_{X_{h_{n}}} \\
& =a\left(\varphi_{n}, \varphi_{n}\right)-\left\|\psi_{n}\right\|_{V_{h_{n}}}^{2}
\end{aligned}
$$

and so, by (4.11) and (4.6), we have

$$
\lim _{n \rightarrow \infty}\left(a\left(\varphi_{n}, \varphi_{n}\right)-\left\|\psi_{n}\right\|_{V_{h_{n}}}^{2}\right)=0
$$

This relation and (4.6) lead to (4.9).

Lemma 4.3. Assume that (4.6) and (4.7) hold. Then there exists $n_{0} \in \mathbb{N}$ such that the sequence $\left(\left|\omega_{n}\right|\right)_{n \geq n_{0}}$ is uniformly bounded away from zero.

Proof. By a contradiction argument, we show that the sequence $\left(\omega_{n}\right)_{n}$ contains no subsequence converging to zero. Namely suppose that such a subsequence exists. For the sake of simplicity, we still denote it by $\left(\omega_{n}\right)_{n}$. Hence (4.11) implies that

$$
-A_{1 h_{n}} z_{n}+\left(\begin{array}{c}
0 \\
h_{n}^{\theta} A_{h_{n}}^{1+\frac{l}{2}} \psi_{n}
\end{array}\right)=\left(\begin{array}{c}
-\left(1+h_{n}^{\theta}\right)^{-1}\left(I+h_{n}^{\theta} A_{h_{n}}^{\frac{l}{2}}\right) \psi_{n} \\
\left(1+h_{n}^{\theta}\right)^{-1}\left(I+h_{n}^{\theta} A_{h_{n}}^{\frac{l}{2}}\right) A_{h_{n}} \varphi_{n}+h_{n}^{\theta} A_{h_{n}}^{1+\frac{l}{2}} \psi_{n}
\end{array}\right) \rightarrow 0 \text { in } X_{h_{n}} .
$$


Taking the inner product of first component in (4.12) with $\psi_{n}$, we get

$$
\left(1+h_{n}^{\theta}\right)^{-1} a\left(\left(I+h_{n}^{\theta} A_{h_{n}}^{\frac{l}{2}}\right) \psi_{n}, \psi_{n}\right)=\left(1+h_{n}^{\theta}\right)^{-1}\left(a\left(\psi_{n}, \psi_{n}\right)+h_{n}^{\theta} a^{l}\left(\psi_{n}, \psi_{n}\right)\right) \rightarrow 0 .
$$

As $h_{n} \leq h^{*}$, then, by (4.8), we get

$$
\left\|A_{h_{n}}^{\frac{1}{2}} \psi_{n}\right\|^{2}=a\left(\psi_{n}, \psi_{n}\right) \rightarrow 0 .
$$

The convergence of the first component in (4.12) implies that

$$
\left\|A_{h_{n}}^{\frac{1}{2}}\left(I+h_{n}^{\theta} A_{h_{n}}^{\frac{l}{2}}\right) \psi_{n}\right\|^{2} \rightarrow 0
$$

Therefore, (4.13) yields

$$
h_{n}^{\theta} A_{h_{n}}^{\frac{(1+l)}{2}} \psi_{n} \rightarrow 0 \text { in } H
$$

The second component in (4.12) and the fact that $\alpha\|x\|^{2} \leq\left\|A_{h}^{\frac{1}{2}} x\right\|^{2}=a(x, x)$ for all $x \in V_{h}$ imply that

$$
\left(1+h_{n}^{\theta}\right)^{-1}\left(I+h_{n}^{\theta} A_{h_{n}}^{\frac{l}{2}}\right) A_{h_{n}}^{\frac{1}{2}} \varphi_{n}+h_{n}^{\theta} A_{h_{n}}^{\frac{1+l}{2}} \psi_{n} \rightarrow 0 \text { in } H,
$$

which, by (4.14), yields

$$
\left(1+h_{n}^{\theta}\right)^{-1}\left(I+h_{n}^{\theta} A_{h_{n}}^{\frac{l}{2}}\right) A_{h_{n}}^{\frac{1}{2}} \varphi_{n} \rightarrow 0 \text { in } H
$$

Thus, as $h_{n} \leq h^{*}$, we get

$$
a\left(\varphi_{n}, \varphi_{n}\right) \rightarrow 0
$$

This above relation and (4.13) contradict (4.6).

According to the above lemma, we note that the coefficient $1+\left|\omega_{n}\right|^{2 l}$ becomes equivalent to $\left|\omega_{n}\right|^{2 l}$. Now, we introduce the operator $D_{1 h_{n}}$ defined by

$$
D_{1 h_{n}}=\left(\begin{array}{cc}
0 & I \\
-A_{h_{n}} & 0
\end{array}\right)
$$

Note that $A_{1 h_{n}}=\left(1+h_{n}^{\theta}\right)^{-1}\left(I+h_{n}^{\theta} A_{h_{n}}^{\frac{l}{2}}\right) D_{1 h_{n}}$. We then use the following spectral basis of the operator $D_{1 h_{n}}$. Namely, we extend the definitions of $\lambda_{k, h_{n}}$ and of $\varphi_{k, h_{n}}$ for $k \in\left\{-1, \ldots,-N\left(h_{n}\right)\right\}$ by setting $\lambda_{k, h_{n}}=-\lambda_{-k, h_{n}}$ and $\varphi_{k, h_{n}}=\varphi_{-k, h_{n}}$. Then an orthonormal basis of $X_{h_{n}}$ formed by the eigenvectors of $D_{1 h_{n}}$ is given by

$$
\Psi_{k, h_{n}}=\frac{1}{\sqrt{2}}\left(\begin{array}{c}
-\frac{i}{\lambda_{k}, h_{n}} \varphi_{k, h_{n}} \\
\varphi_{k, h_{n}}
\end{array}\right), 0<|k| \leq N\left(h_{n}\right)
$$

of associated eigenvalue $i \lambda_{k, h_{n}}$, that is to say

$$
D_{1 h_{n}} \Psi_{k, h_{n}}=i \lambda_{k, h_{n}} \Psi_{k, h_{n}} .
$$

Consequently, for all $n \in \mathbb{N}$, there exist complex coefficients $\left(c_{k}^{n}\right)_{0<|k| \leq N\left(h_{n}\right)}$ such that

$$
z_{n}=\sum_{0<|k| \leq N\left(h_{n}\right)} c_{k}^{n} \Psi_{k, h_{n}} .
$$

The normalization condition (4.6) implies that

$$
\sum_{0<|k| \leq N\left(h_{n}\right)}\left|c_{k}^{n}\right|^{2}=1 .
$$


Let $\epsilon$ be the constant from Proposition 2.4 (if $l=0$, we recover the condition from Prop. 2.1). For any $n \in \mathbb{N}$, we define

$$
M_{l}\left(h_{n}\right)=\max \left\{k \in\left\{1, \ldots, N\left(h_{n}\right)\right\} \mid h_{n}^{\theta}\left(\lambda_{k}\right)^{2} \leq \frac{\epsilon}{\lambda_{k}^{l}}\right\}
$$

if $h_{n}^{\theta}\left(\lambda_{1}\right)^{2} \leq \frac{\epsilon}{\lambda_{1}^{\nu}}$ and $M_{l}\left(h_{n}\right)=0$ otherwise.

Lemma 4.4. Suppose that the sequences $\left(h_{n}\right),\left(\omega_{n}\right),\left(z_{n}\right)$ satisfy (4.6) and (4.7). Then, we have

$$
\begin{gathered}
\psi_{n}=\frac{1}{\sqrt{2}} \sum_{k=1}^{N\left(h_{n}\right)}\left(c_{k}^{n}+c_{-k}^{n}\right) \varphi_{k, h_{n}}, \\
\sum_{M_{l}\left(h_{n}\right)<k \leq N\left(h_{n}\right)}\left|\omega_{n}\right|^{2 l}\left|c_{k}^{n}+c_{-k}^{n}\right|^{2} \rightarrow 0,
\end{gathered}
$$

and

$$
\sum_{0<|k| \leq M_{l}\left(h_{n}\right)}\left|\omega_{n}\right|^{2 l}\left|\omega_{n}-\left(1+h_{n}^{\theta}\right)^{-1}\left(\lambda_{k, h_{n}}+h_{n}^{\theta} \lambda_{k, h_{n}}^{1+l}\right)\right|^{2}\left|c_{k}^{n}\right|^{2} \rightarrow 0 .
$$

Proof. Relation (4.18) follows directly by taking the second component in (4.16) and by using (4.15) and the fact that $\varphi_{k, h}=\varphi_{-k, h}$.

On the other hand, we use (4.16) and the fact that $\Psi_{k, h_{n}}$ is an eigenvector of $D_{1 h_{n}}$ associated with eigenvalue $i \lambda_{k, h_{n}}$ to obtain for all $\tilde{\psi}_{h_{n}} \in X_{h_{n}}$

$$
\left(i \omega_{n} z_{n}-A_{1 h_{n}} z_{n}, \tilde{\psi}_{h_{n}}\right)_{X_{h_{n}}}=\sum_{0<|k| \leq N\left(h_{n}\right)} i\left(\omega_{n}-\left(1+h_{n}^{\theta}\right)^{-1}\left(\lambda_{k, h_{n}}+h_{n}^{\theta} \lambda_{k, h_{n}}^{1+l}\right)\right) c_{k}^{n}\left(\Psi_{k, h_{n}}, \tilde{\psi}_{h_{n}}\right)_{X_{h_{n}}} .
$$

From (4.8) and (4.18), it follows that

$$
\left|\omega_{n}\right|^{2 l} h_{n}^{\theta} a^{l}\left(\psi_{n}, \psi_{n}\right)=\frac{1}{2} \sum_{k=1}^{N\left(h_{n}\right)} h_{n}^{\theta} \lambda_{k, h_{n}}^{2+l}\left|\omega_{n}\right|^{2 l}\left|c_{k}^{n}+c_{-k}^{n}\right|^{2} \rightarrow 0 .
$$

As we have $\lambda_{k} \leq \lambda_{k, h_{n}}$ for all $k \in\left\{1, \ldots, N\left(h_{n}\right)\right\}$ and by the definition (4.17), we obtain (4.19).

By (2.9), we have

$$
h_{n}^{\theta} \lambda_{k, h_{n}}^{2} \leq h_{n}^{\theta}\left(\lambda_{k}+\left(C h_{n}^{\theta} \lambda_{k}^{2}\right)^{2}\right)^{2} \leq 2 h_{n}^{\theta} \lambda_{k}^{2}+2 C^{4} h_{n}^{\theta}\left(h_{n}^{\theta} \lambda_{k}^{2}\right)^{4} \leq C \frac{\epsilon}{\lambda_{k}^{l}}+C \frac{\epsilon^{4}}{\lambda_{k}^{4 l}} \leq C^{\prime} \frac{\epsilon}{\lambda_{k}^{l}}
$$

for $h_{n}^{\theta}\left(\lambda_{k}\right)^{2} \leq \frac{\epsilon}{\lambda_{k}^{l}}$. So, by using (4.22) and again (2.9), there exists a constant $C$ independent of $h_{n}$ such that

$$
\begin{aligned}
h_{n}^{2 \theta} \sum_{k=1}^{M_{l}\left(h_{n}\right)} \lambda_{k, h_{n}}^{4+2 l}\left|\omega_{n}\right|^{2 l}\left|c_{k}^{n}+c_{-k}^{n}\right|^{2} & \leq C \sum_{k=1}^{M_{l}\left(h_{n}\right)} \epsilon h_{n}^{\theta} \lambda_{k, h_{n}}^{2+l}\left|\omega_{n}\right|^{2 l}\left|c_{k}^{n}+c_{-k}^{n}\right|^{2} \\
& \leq C \epsilon \sum_{k=1}^{M_{l}\left(h_{n}\right)} h_{n}^{\theta} \lambda_{k, h_{n}}^{2+l}\left|\omega_{n}\right|^{2 l}\left|c_{k}^{n}+c_{-k}^{n}\right|^{2} \rightarrow 0 .
\end{aligned}
$$

We also have for all $\tilde{\psi}_{h_{n}} \in X_{h_{n}}$

$$
\left(\left(\begin{array}{c}
0 \\
h_{n}^{\theta} A_{h_{n}}^{1+\frac{l}{2}} \psi_{n}
\end{array}\right), \tilde{\psi}_{h_{n}}\right)_{X_{h_{n}}}=\sum_{0<|k| \leq N\left(h_{n}\right)} \frac{h_{n}^{\theta}}{2} \lambda_{k, h_{n}}^{2+l}\left(c_{k}^{n}+c_{-k}^{n}\right)\left(\Psi_{k, h_{n}}, \tilde{\psi}_{h_{n}}\right)_{X_{h_{n}}}
$$


because $l$ is even. Relations (4.24) and (4.25) imply that for all $\tilde{\psi}_{h_{n}} \in X_{h_{n}}$

$$
\left|\omega_{n}\right|^{l}\left(\left(\begin{array}{c}
0 \\
h_{n}^{\theta} A_{h_{n}}^{1+\frac{l}{2}} \psi_{n}
\end{array}\right)-\sum_{M_{l}\left(h_{n}\right)<|k| \leq N\left(h_{n}\right)} \frac{h_{n}^{\theta}}{2} \lambda_{k, h_{n}}^{2+l}\left(c_{k}^{n}+c_{-k}^{n}\right) \Psi_{k, h_{n}}, \tilde{\psi}_{h_{n}}\right)_{X_{h_{n}}} \rightarrow 0 .
$$

So, we obtain with (4.11), (4.21) and the above relation, for all $\tilde{\psi}_{h_{n}} \in X_{h_{n}}$, that the inner product in $X_{h_{n}}$ of $\tilde{\psi}_{h_{n}}$ with

$$
\begin{aligned}
& \quad \sum_{0<|k| \leq N\left(h_{n}\right)} i\left|\omega_{n}\right|^{l}\left(\omega_{n}-\left(1+h_{n}^{\theta}\right)^{-1}\left(\lambda_{k, h_{n}}+h_{n}^{\theta} \lambda_{k, h_{n}}^{1+l}\right)\right) c_{k}^{n} \Psi_{k, h_{n}} \\
& +\sum_{M_{l}\left(h_{n}\right)<|k| \leq N\left(h_{n}\right)} \frac{h_{n}^{\theta}}{2}\left|\omega_{n}\right|^{l} \lambda_{k, h_{n}}^{2+l}\left(c_{k}^{n}+c_{-k}^{n}\right) \Psi_{k, h_{n}}
\end{aligned}
$$

tends to zero. Taking $\tilde{\psi}_{h_{n}} \in X_{h_{n}}$ to be equal to the same above relation and as the family $\left(\Psi_{k, h_{n}}\right)$ is orthogonal, the above relation implies (4.20).

\section{Proof of Theorem 1.2}

We use the results of the previous section with $l=0$ and set, for shortness, $\tilde{A}_{h}:=\tilde{A}_{0, h}$ and $M\left(h_{n}\right):=M_{0}\left(h_{n}\right)$.

Proof of Theorem 1.2. This proof is based on Theorem 3.1. First, for all $h \in\left(0, h^{*}\right)$, the family ( $\left.\mathrm{e}^{t \tilde{A}_{h}}\right)$ forms a contraction semigroup. The family $\left(\tilde{A}_{h}\right)$ satisfies the condition i) in Theorem 3.1 owing to Lemma 4.1. To show that the family $\left(\tilde{A}_{h}\right)$ also satisfies the condition ii) in Theorem 3.1 , we use a contradiction argument. Let $\left(h_{n}\right)_{n},\left(\omega_{n}\right)_{n}$ and $z_{n}=\left(\begin{array}{l}\varphi_{n} \\ \psi_{n}\end{array}\right) \in \mathcal{D}\left(\tilde{A}_{h_{n}}\right)$ be three sequences satisfying (4.6) and (4.7). Notice that for $k_{m} \in A_{k}$, we have

$$
\begin{aligned}
\lambda_{k_{m}, h_{n}}-\lambda_{k_{m-1}+l_{m-1}-1, h_{n}} & \geq \lambda_{k_{m}}-\lambda_{k_{m-1}+l_{m-1}-1}-c \epsilon \\
& =\lambda_{k_{m}}-\lambda_{k_{m-1}}-c \epsilon \geq \gamma_{0}^{\prime}-c \epsilon \\
& \geq \frac{\gamma_{0}^{\prime}}{2}=: \gamma^{\prime}
\end{aligned}
$$

for $\epsilon \leq \frac{\gamma_{0}^{\prime}}{2 c}$ by (2.8) and (2.9). We now introduce the set

$$
\begin{aligned}
\mathcal{F}=\left\{n \in \mathbb{N}\left|\exists k(n) \in\{1, \ldots, M\}, \exists k_{m(n)} \in A_{k(n)},\right| k_{m(n)} \mid \leq M\left(h_{n}\right)\right. \text { and } \\
\\
\left.\qquad k_{m(n)+k(n)-1}+l_{m(n)+k(n)-1} \mid \leq N\left(h_{n}\right) \text { such that }\left|\omega_{n}-\lambda_{k_{m(n)}, h_{n}}\right|<\frac{\gamma^{\prime}}{2}\right\} .
\end{aligned}
$$

We distinguish two cases.

First case. The set $\mathcal{F}$ is infinite. Then, without loss of generality, we can suppose that $\mathcal{F}=\mathbb{N}$ (otherwise we take a subsequence of $\left.\left(\omega_{n}\right)\right)$. Then, by reducing the value of $\gamma^{\prime}$ if needed, we can assume that for all $n \in \mathbb{N}$, we have that for all $k_{m} \in A_{k^{\prime}}, k^{\prime}=1, \ldots, M$ with $m \neq m(n)$,

$$
\left|\omega_{n}-\lambda_{k_{m+j}+l, h_{n}}\right| \geq \frac{\gamma^{\prime}}{2}, \forall j=0, \ldots, k^{\prime}-1, \forall l=0, \ldots, l_{m+j}-1 .
$$

By using (4.20), we obtain that

$$
\begin{aligned}
& \sum_{k=1}^{M} \sum_{k_{m} \in A_{k}} \sum_{j=0}^{k-1} \sum_{l=0}^{l_{m+j}-1} \quad\left|c_{k_{m+j}+l}^{n}\right|^{2} \rightarrow 0 . \\
& m \neq m(n) \quad 0<\left|k_{m+j}+l_{m+j}-1\right| \leq M\left(h_{n}\right)
\end{aligned}
$$


Define now

$$
\tilde{\psi}_{n}=\frac{1}{\sqrt{2}} \sum_{j=0}^{k(n)-1 l_{m(n)+j}-1} \sum_{l=0}^{n} c_{k_{m(n)+j}+l}^{n} \varphi_{k_{m(n)+j}+l, h_{n}} .
$$

We have, by (4.18),

$$
\psi_{n}=\frac{1}{\sqrt{2}} \sum_{k=1}^{M} \sum_{k_{m} \in A_{k}} \sum_{\substack{j=0 \\ 1 \leq k_{m+j}+l \leq N\left(h_{n}\right)}}^{k-1} \sum_{l=0}^{l_{m+j}-1}\left(c_{k_{m+j}+l}^{n}+c_{-\left(k_{m+j}+l\right)}^{n}\right) \varphi_{k_{m+j}+l, h_{n}},
$$

and so, by (5.2) and (4.19), we obtain

$$
\left\|\tilde{\psi}_{n}-\psi_{n}\right\| \rightarrow 0
$$

Thus, since $\left(\left\|B_{h}^{*}\right\|_{\mathcal{L}\left(V_{h}, U\right)}\right)_{h \in\left(0, h^{*}\right)}$ is bounded, we deduce that

$$
\left\|B_{h_{n}}^{*}\left(\tilde{\psi}_{n}-\psi_{n}\right)\right\|_{U} \rightarrow 0
$$

The above relation and (4.8) imply that

$$
\left\|B_{h_{n}}^{*} \tilde{\psi}_{n}\right\|_{U} \rightarrow 0
$$

But

$$
\begin{aligned}
\left\|B_{h_{n}}^{*} \tilde{\psi}_{n}\right\|_{U} & =\frac{1}{\sqrt{2}}\left\|\sum_{j=0}^{\|(n)-1 l_{m(n)+j}-1} \sum_{l=0}^{k} c_{k_{m(n)+j}+l}^{n} B_{h_{n}}^{*} \varphi_{k_{m(n)+j}+l, h_{n}}\right\|_{U} \\
& =\frac{1}{\sqrt{2}}\left\|\left(B_{h_{n}}^{*} \varphi_{k_{m(n)}, h_{n}} \cdots B_{h_{n}}^{*} \varphi_{k_{m(n)+k(n)-1}+l_{m(n)+k(n)-1}-1, h_{n}}\right) C\right\|_{U} \\
& =\frac{1}{\sqrt{2}}\left\|(1 \cdots 1) \Phi_{k_{m(n)}, h_{n}} C\right\|_{U},
\end{aligned}
$$

where $C=\left(c_{k_{m(n)}} \cdots c_{k_{m(n)}+l_{m(n)}-1} c_{k_{m(n)+1}} \cdots c_{k_{m(n)+k(n)-1}+l_{m(n)+k(n)-1}-1}\right)^{T}$. So, we have

$$
\left\|B_{h_{n}}^{*} \tilde{\psi}_{n}\right\|_{U}=\frac{1}{\sqrt{2}}\left\|\left(\begin{array}{cccc}
1 & 1 & \cdots & 1 \\
0 & 0 & \cdots & 0 \\
\vdots & \ddots & \vdots \\
0 & \cdots & 0
\end{array}\right) \Phi_{k_{m(n)}, h_{n}} C\right\|_{U, 2} .
$$

We now use Lemma 1.1 to have

$$
\begin{aligned}
\left\|B_{h_{n}}^{*} \tilde{\psi}_{n}\right\|_{U} & \geq c\left\|B_{k_{m(n)}}^{-1} \Phi_{k_{m(n)}, h_{n}} C\right\|_{U, 2} \text { for } n \text { large enough } \\
& \geq c \alpha\|C\|_{2} \text { by Proposition } 2.1 .
\end{aligned}
$$

Gathering (5.3)-(5.6), we obtain that $\tilde{\psi}_{n} \rightarrow 0$ in $H$. Therefore, by (5.4), $\psi_{n} \rightarrow 0$, which contradicts (4.9).

Second case. the set $\mathcal{F}$ is finite. Then, we can assume, without loss of generality, that $\mathcal{F}$ is empty (otherwise we take off the finite number of $\left.\left(\omega_{n}\right)\right)$, i.e., that for all $n \in \mathbb{N}$, we have that

$$
\left|\omega_{n}-\lambda_{k, h_{n}}\right| \geq \frac{\gamma^{\prime}}{2} \quad \text { if } 0<|k| \leq M\left(h_{n}\right) .
$$

Thus, by (4.20) and the above relation, we obtain that

$$
\sum_{0<|k| \leq M\left(h_{n}\right)}\left|c_{k}^{n}\right|^{2} \rightarrow 0 .
$$

Therefore, by (4.18), (4.19) and the above relation, we have $\psi_{n} \rightarrow 0$ in $H$, which contradicts (4.9).

In conclusion, the family $\left(\tilde{A}_{h}\right)$ satisfies the condition (ii) in Theorem 3.1 and so the family of systems (1.9) is uniformly exponentially stable. 


\section{Proof of Theorem 1.6}

Here we use the results of Section 4 with $l>0$ and $l$ even. Without loss of generality, we may assume that $0<h<h^{*}=1$.

Proof of Theorem 1.6 and of (3.2) This proof is based on Theorem 3.8. First, for all $h \in\left(0, h^{*}\right),\left(\mathrm{e}^{t \tilde{A}_{l, h}}\right)$ forms a family of contraction semigroups and the family $\left(\tilde{A}_{l, h}\right)_{h}$ satisfies (3.1). To apply the results of Theorem 3.8, the family $\left(\tilde{A}_{l, h}\right)$ must also satisfy condition i) of Theorem 3.8 with $\alpha=2 l$ and condition (3.2) or equivalently condition (4.5). We again use a contradiction argument to prove this last condition. Let $\left(h_{n}\right)_{n},\left(\omega_{n}\right)_{n}$ and $z_{n}=\left(\begin{array}{c}\varphi_{n} \\ \psi_{n}\end{array}\right) \in X_{h}$ be three sequences satisfying (4.6) and (4.7). Notice that for $k_{m} \in A_{k}$, we have

$$
\begin{aligned}
\lambda_{k_{m}, h}-\lambda_{k_{m-1}+l_{m-1}-1, h} & \geq \lambda_{k_{m}}-\lambda_{k_{m-1}+l_{m-1}-1}-\frac{c \epsilon}{\lambda_{k_{m}-1}^{2 l}} \\
& \geq \lambda_{k_{m}}-\lambda_{k_{m-1}}-\frac{c \epsilon}{\lambda_{k_{1}}^{2 l}} \geq \gamma_{0}^{\prime}-\frac{c \epsilon}{\lambda_{k_{1}}^{2 l}} \\
& \geq \frac{\gamma_{0}^{\prime}}{2}=: \gamma^{\prime}
\end{aligned}
$$

for $\epsilon \leq \frac{\gamma_{0}^{\prime} \lambda_{k_{1}}^{2 l}}{2 c}$ by (2.8), (2.9) and because $\lambda_{k_{m}} \geq \lambda_{k_{1}}>0$. We introduce the set $\mathcal{F}_{2}$ like

$$
\begin{aligned}
& \mathcal{F}_{2}=\left\{n \in \mathbb{N}\left|\exists k(n) \in\{1, \ldots, M\}, \exists k_{m(n)} \in A_{k(n)},\right| k_{m(n)} \mid \leq M_{l}\left(h_{n}\right)\right. \text { and } \\
& \left.\left|k_{m(n)+k(n)-1}+l_{m(n)+k(n)-1}\right| \leq N\left(h_{n}\right) \text { such that }\left|\omega_{n}-\left(1+h_{n}^{\theta}\right)^{-1}\left(\lambda_{k_{m(n)}, h_{n}}+h_{n}^{\theta} \lambda_{k_{m(n)}, h_{n}}^{1+l}\right)\right|<\frac{\gamma^{\prime}}{4}\right\} .
\end{aligned}
$$

We distinguish two cases.

First case. the set $\mathcal{F}_{2}$ is infinite. Then, without loss of generality, we can suppose that $\mathcal{F}_{2}=\mathbb{N}$ (otherwise we take a subsequence of $\left.\left(\omega_{n}\right)_{n}\right)$. Then, by reducing the value of $\gamma^{\prime}$ if needed, we can assume that for all $n \in \mathbb{N}$, we have that for all $k_{m} \in A_{k^{\prime}}, k^{\prime}=1, \ldots, M$ with $m \neq m(n)$, and for all $\left|k_{m+j}+l\right| \leq M_{l}\left(h_{n}\right)$

$$
\left|\omega_{n}-\left(1+h_{n}^{\theta}\right)^{-1}\left(\lambda_{k_{m+j}+l, h_{n}}+h_{n}^{\theta} \lambda_{k_{m+j}+l, h_{n}}^{1+l}\right)\right| \geq \frac{\gamma^{\prime}}{8}, \forall j=0, \ldots, k^{\prime}-1, \forall l=0, \ldots, l_{m+j}-1 .
$$

Indeed, similar to (4.23), we have

$$
\begin{aligned}
\left|\omega_{n}-\left(1+h_{n}^{\theta}\right)^{-1}\left(\lambda_{k_{m+j}+l, h_{n}}+h_{n}^{\theta} \lambda_{k_{m+j}+l, h_{n}}^{1+l}\right)\right| \geq & \left(1+h_{n}^{\theta}\right)^{-1}\left|\lambda_{k_{m+j}+l, h_{n}}-\lambda_{k_{m(n)}, h_{n}}\right| \\
& -\left|\omega_{n}-\left(1+h_{n}^{\theta}\right)^{-1}\left(\lambda_{k_{m(n)}, h_{n}}+h_{n}^{\theta} \lambda_{k_{m(n)}, h_{n}}^{1+l}\right)\right| \\
& -\left(1+h_{n}^{\theta}\right)^{-1}\left(h_{n}^{\theta} \lambda_{k_{m(n)}, h_{n}}^{1+l}+h_{n}^{\theta} \lambda_{k_{m+j}+l, h_{n}}^{1+l}\right) \\
& \geq \frac{\gamma^{\prime}}{2}-\frac{\gamma^{\prime}}{4}-\frac{2 C \epsilon}{\lambda_{k_{1}}} .
\end{aligned}
$$

So choose again $\epsilon \leq \frac{\gamma^{\prime} \lambda_{k_{1}}}{16 C}$ to get (6.2). By using (4.20), we obtain that

$$
\sum_{k=1}^{M} \sum_{\substack{k_{m} \in A_{k} \\ m \neq m(n)}} \sum_{\substack{l=0 \\ m \neq m}}^{k-1} \sum_{\substack{l=0 \\ l_{m+j}-1}}\left|\omega_{n}\right|^{2 l}\left|c_{k_{m+j}+l}^{n}\right|^{2} \rightarrow 0 .
$$

Define now

$$
\tilde{\psi}_{n}=\frac{1}{\sqrt{2}} \sum_{j=0}^{k(n)-1 l_{m(n)+j}-1} \sum_{l=0}^{n} c_{k_{m(n)+j}+l}^{n} \varphi_{k_{m(n)+j}+l, h_{n}} .
$$


We have, by (4.18),

$$
\psi_{n}=\frac{1}{\sqrt{2}} \sum_{k=1}^{M} \sum_{k_{m} \in A_{k}} \sum_{\substack{j=0 \\ 1 \leq k_{m+j}+l \leq N\left(h_{n}\right)}}^{k-1} \sum_{l=0}^{l_{m+j}-1}\left(c_{k_{m+j}+l}^{n}+c_{-\left(k_{m+j}+l\right)}^{n}\right) \varphi_{k_{m+j}+l, h_{n}},
$$

and so, by (6.3) and (4.19), we obtain

$$
\left|\omega_{n}\right|^{l}\left\|\tilde{\psi}_{n}-\psi_{n}\right\| \rightarrow 0
$$

Thus, since $\left(\left\|B_{h}^{*}\right\|_{\mathcal{L}\left(V_{h}, U\right)}\right)_{h \in\left(0, h^{*}\right)}$ is bounded, we deduce that

$$
\left|\omega_{n}\right|^{l}\left\|B_{h_{n}}^{*}\left(\tilde{\psi}_{n}-\psi_{n}\right)\right\|_{U} \rightarrow 0 .
$$

The above relation and (4.8) imply that

$$
\left|\omega_{n}\right|^{l}\left\|B_{h_{n}}^{*} \tilde{\psi}_{n}\right\|_{U} \rightarrow 0
$$

But

$$
\begin{aligned}
& \left|\omega_{n}\right|^{l}\left\|B_{h_{n}}^{*} \tilde{\psi}_{n}\right\|_{U}=\frac{\left|\omega_{n}\right|^{l}}{\sqrt{2}}\left\|\sum_{j=0}^{k(n)-1 l_{m(n)+j}-1} \sum_{l=0}^{n} c_{k_{m(n)+j}+l}^{n} B_{h_{n}}^{*} \varphi_{k_{m(n)+j}+l, h_{n}}\right\|_{U} \\
& =\frac{\left|\omega_{n}\right|^{l}}{\sqrt{2}}\left\|\left(B_{h_{n}}^{*} \varphi_{k_{m(n)}, h_{n}} \cdots B_{h_{n}}^{*} \varphi_{k_{m(n)+k(n)-1}+l_{m(n)+k(n)-1}-1, h_{n}}\right) C\right\|_{U} \\
& =\frac{\left|\omega_{n}\right|^{l}}{\sqrt{2}}\left\|(1 \cdots 1) \Phi_{k_{m(n)}, h_{n}} C\right\|_{U} \text {, }
\end{aligned}
$$

where $C=\left(c_{k_{m(n)}} \cdots c_{k_{m(n)}+l_{m(n)}-1} c_{k_{m(n)+1}} \cdots c_{k_{m(n)+k(n)-1}+l_{m(n)+k(n)-1}-1}\right)^{T}$. So, we have

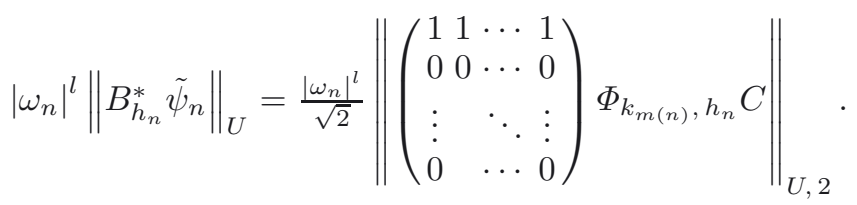

We now use Lemma 1.1 to have

$$
\begin{aligned}
\left|\omega_{n}\right|^{l}\left\|B_{h_{n}}^{*} \tilde{\psi}_{n}\right\|_{U} & \geq c\left|\omega_{n}\right|^{l}\left\|B_{k_{m(n)}}^{-1} \Phi_{k_{m(n)}, h_{n}} C\right\|_{U, 2} \text { for } n \text { large enough } \\
& \geq c \frac{\alpha\left|\omega_{n}\right|^{l}}{\lambda_{k_{m(n)}}^{l}}\|C\|_{2} \text { by Proposition 2.4. }
\end{aligned}
$$

But, $\omega_{n}$ verifies $\left|\omega_{n}-\left(1+h_{n}^{\theta}\right)^{-1}\left(\lambda_{k_{m(n)}, h_{n}}+h_{n}^{\theta} \lambda_{k_{m(n)}, h_{n}}^{1+l}\right)\right|<\frac{\gamma^{\prime}}{4}$ by definition (6.1) of $\mathcal{F}_{2}$, thus $\left|\omega_{n}\right| \geq(1+$ $\left.h_{n}^{\theta}\right)^{-1}\left(\lambda_{k_{m(n)}, h_{n}}+h_{n}^{\theta} \lambda_{k_{m(n)}, h_{n}}^{1+l}\right)-\frac{\gamma^{\prime}}{4} \geq \frac{1}{2} \lambda_{k_{m(n)}, h_{n}}-\frac{\gamma^{\prime}}{4}$. Therefore, we have

$$
\begin{aligned}
\left|\omega_{n}\right|^{l}\left\|B_{h_{n}}^{*} \tilde{\psi}_{n}\right\|_{U} & \geq \frac{c \alpha}{2^{l}} \frac{\left(\lambda_{k_{m(n)}, h_{n}}-\frac{\gamma^{\prime}}{2}\right)^{l}}{\lambda_{k_{m(n)}}^{l}}\|C\|_{2} \\
& \geq \frac{c \alpha}{2^{2 l}} \frac{\lambda_{k_{m(n)}, h_{n}}^{l}\|C\|_{2} \text { for } n \text { large enough }}{\lambda_{k_{m(n)}}^{l}} \\
& \geq \frac{c \alpha}{2^{2 l}}\|C\|_{2} \text { by }(2.8) .
\end{aligned}
$$

Gathering (6.4)-(6.7), we obtain that $\tilde{\psi}_{n} \rightarrow 0$ in $H$. Therefore, by (6.5), $\psi_{n} \rightarrow 0$, which contradicts (4.9). 
Second case. the set $\mathcal{F}_{2}$ is finite. We proceed similar to the proof of the second case of Theorem 1.2.

In conclusion, the family $\left(\tilde{A}_{l, h}\right)$ satisfies $(4.5)$; i.e., the condition (i) in Theorem 3.8 with $\alpha=2 l$ when $l$ is even and property (3.2) of Section 3.2.

\section{A CONVERGEnCE RESUlt}

Here we want to prove that the solution $\omega_{h}$ of the discrete problem (1.14) tends to the solution $\omega$ of the continuous problem (1.1) in $X:=V \times H$ as $h$ goes to zero and if the discrete initial data are well chosen. This is obtained with the help of a general version of the Trotter-Kato Theorem proved in [23] that is appropriated when the approximated semi-groups are defined in proper subspaces of the limit one. The basic idea is that the convergence of the semi-groups is equivalent to the convergence of the resolvent, hence we prove such a convergence result for the resolvents.

Before going on we recall that (1.1) is equivalent to

$$
\dot{z}(t)=\tilde{A} z(t) \text { in } X, \quad z(0)=\left(\omega_{0}, \omega_{1}\right)^{\top},
$$

where $z(t)=(\omega(t), \dot{\omega}(t))^{\top}$ and

$$
\tilde{A}\left(\begin{array}{l}
u \\
v
\end{array}\right)=\left(\begin{array}{l}
v \\
-A u-B B^{*} v
\end{array}\right)=\left(\begin{array}{ll}
0 & I \\
-A-B B^{*}
\end{array}\right)\left(\begin{array}{l}
u \\
v
\end{array}\right) .
$$

It is easy to check that $\tilde{A}$ with domain $\mathcal{D}(\tilde{A})=\mathcal{D}(A) \times V$ is a maximal dissipative operator in $X$, equipped with the inner product

$$
\left((u, v)^{\top},\left(u^{*}, v^{*}\right)^{\top}\right)_{X}=a\left(u, u^{*}\right)+\left(v, v^{*}\right) \quad \forall(u, v)^{\top},\left(u^{*}, v^{*}\right)^{\top} \in X .
$$

Moreover, $\tilde{A}$ has no eigenvalues on the imaginary axis. We will denote by $T(t), t \geq 0$ the strongly continuous semi-group of contractions generated by $\tilde{A}$.

Let us start with some preliminary results.

Lemma 7.1. Let $l \in \mathbb{N}, l \geq 2$. If $f \in V=\mathcal{D}\left(A^{\frac{1}{2}}\right)$, then

$$
\left\|\left(1+h^{\theta}\right)\left(I+h^{\theta} A_{h}^{\frac{l}{2}}\right)^{-1} \pi_{h} f-\pi_{h} f\right\|_{H} \leq C h^{\frac{\theta}{l}}\|f\|_{V},
$$

for some $C>0$.

Proof. We write

$$
\pi_{h} f=\sum_{k=1}^{N(h)} f_{k} \varphi_{k, h}
$$

with $f_{k} \in \mathbb{C}$. Hence

$$
v_{h}=\left(1+h^{\theta}\right)\left(I+h^{\theta} A_{h}^{\frac{l}{2}}\right)^{-1} \pi_{h} f,
$$

can be written

$$
v_{h}=\sum_{k=1}^{N(h)} v_{k} \varphi_{k, h},
$$


with $v_{k}=\left(1+h^{\theta}\right)\left(1+h^{\theta} \lambda_{k, h}^{l}\right)^{-1} f_{k}$. Consequently we have

$$
\begin{aligned}
\left\|v_{h}-\pi_{h} f\right\|_{H}^{2} & =\sum_{k=1}^{N(h)}\left|f_{k}\right|^{2}\left(\left(1+h^{\theta}\right)\left(1+h^{\theta} \lambda_{k, h}^{l}\right)^{-1}-1\right)^{2} \\
& =h^{2 \theta} \sum_{k=1}^{N(h)}\left|f_{k}\right|^{2}\left(\frac{1-\lambda_{k, h}^{l}}{1+h^{\theta} \lambda_{k, h}^{l}}\right)^{2} \\
& \leq \operatorname{ch}^{2 \theta} \sum_{k=1}^{N(h)}\left|f_{k}\right|^{2} \frac{\lambda_{k, h}^{2 l}}{\left(1+h^{\theta} \lambda_{k, h}^{l}\right)^{2}} \\
& \leq \operatorname{ch}^{2 \theta} \sum_{k=1}^{N(h)} \lambda_{k, h}^{2}\left|f_{k}\right|^{2}\left(g\left(\lambda_{k, h}\right)\right)^{2}
\end{aligned}
$$

for some $c>0$ independent of $h$, where the function $g:[0, \infty) \mapsto \mathbb{R}$ is given by $g(\lambda)=\frac{\lambda^{l-1}}{\left(1+h^{\theta} \lambda^{l}\right)}$. As the maximum of $g$ is attained at $\lambda_{0}>0$ given by

$$
h^{\theta} \lambda_{0}^{l}=l-1,
$$

we get that

$$
\left\|v_{h}-\pi_{h} f\right\|_{H}^{2} \leq c c_{2}^{2} h^{\frac{2 \theta}{l}} \sum_{k=1}^{N(h)}\left|f_{k}\right|^{2} \lambda_{k, h}^{2}
$$

since $\lambda_{0}=c_{1} h^{-\frac{\theta}{l}}$ and $g\left(\lambda_{0}\right)=c_{2} h^{-\frac{\theta(l-1)}{l}}$ with $c_{1}, c_{2}$ two positive constants independent of $h$. This proves the first estimate since

$$
\sum_{k=1}^{N(h)}\left|f_{k}\right|^{2} \lambda_{k, h}^{2}=\left\|A_{h}^{\frac{1}{2}} \pi_{h} f\right\|_{H}^{2}=a\left(\pi_{h} f, \pi_{h} f\right) \leq a(f, f)=\left\|A^{\frac{1}{2}} f\right\|_{H}^{2} .
$$

Corollary 7.2. Let $l \in \mathbb{N}, l \geq 2$, then for any $f_{h} \in V_{h}$ we have

$$
\left\|\left(1+h^{\theta}\right)\left(I+h^{\theta} A_{h}^{\frac{l}{2}}\right)^{-1} f_{h}-f_{h}\right\|_{\mathcal{D}\left(A_{h}^{-\frac{1}{2}}\right)} \leq C h^{\frac{\theta}{l}}\left\|f_{h}\right\|_{H},
$$

for some $C>0$.

Proof. As in the previous lemma, we have

$$
\begin{aligned}
\left\|\left(1+h^{\theta}\right)\left(I+h^{\theta} A_{h}^{\frac{l}{2}}\right)^{-1} f_{h}-f_{h}\right\|_{\mathcal{D}\left(A_{h}^{-\frac{1}{2}}\right)}^{2} & =\left\|A_{h}^{-\frac{1}{2}}\left(\left(1+h^{\theta}\right)\left(I+h^{\theta} A_{h}^{\frac{l}{2}}\right)^{-1} f_{h}-f_{h}\right)\right\|_{H}^{2} \\
& =h^{2 \theta} \sum_{k=1}^{N(h)} \lambda_{k, h}^{-2}\left|f_{k}\right|^{2}\left(\frac{1-\lambda_{k, h}^{l}}{1+h^{\theta} \lambda_{k, h}^{l}}\right)^{2} \\
& \leq c h^{2 \theta} \sum_{k=1}^{N(h)}\left|f_{k}\right|^{2}\left(g\left(\lambda_{k, h}\right)\right)^{2},
\end{aligned}
$$

when

$$
f_{h}=\sum_{k=1}^{N(h)} f_{k} \varphi_{k, h}
$$

We then conclude as before. 
Lemma 7.3. Let $l \in \mathbb{N}, l \geq 2$ and let $f \in \mathcal{D}(A)$, then

$$
h^{\theta}\left\|A_{h}^{1+\frac{l}{2}}\left(I+h^{\theta} A_{h}^{\frac{l}{2}}\right)^{-2} \pi_{h} f\right\|_{\mathcal{D}\left(A_{h}^{-\frac{1}{2}}\right)} \leq C h^{\frac{\theta}{T}}\|f\|_{\mathcal{D}(A)}
$$

for some $C>0$.

Proof. We easily see that

$$
\begin{aligned}
h^{2 \theta}\left\|A_{h}^{1+\frac{l}{2}}\left(I+h^{\theta} A_{h}^{\frac{l}{2}}\right)^{-2} \pi_{h} f\right\|_{\mathcal{D}\left(A_{h}^{-\frac{1}{2}}\right)}^{2} & =h^{2 \theta}\left\|A_{h}^{-\frac{1}{2}} A_{h}^{1+\frac{l}{2}}\left(I+h^{\theta} A_{h}^{\frac{l}{2}}\right)^{-2} \pi_{h} f\right\|_{H}^{2} \\
& =h^{2 \theta} \sum_{k=1}^{N(h)}\left|f_{k}\right|^{2} \frac{\lambda_{k, h}^{2 l+2}}{\left(1+h^{\theta} \lambda_{k, h}^{l}\right)^{4}} \\
& \leq h^{2 \theta} \sum_{k=1}^{N(h)}\left|f_{k}\right|^{2} \lambda_{k, h}^{4}\left(g\left(\lambda_{k, h}\right)\right)^{2}
\end{aligned}
$$

and we conclude as before.

Lemma 7.4. Let $l \in \mathbb{N}, l \geq 2$ and let $f \in V$, then

$$
\left\|\left(1+h^{\theta}\right)\left(I+h^{\theta} A_{h}^{\frac{l}{2}}\right)^{-1} B_{h} B_{h}^{*}\left(1+h^{\theta}\right)\left(I+h^{\theta} A_{h}^{\frac{l}{2}}\right)^{-1} \pi_{h} f-B_{h} B_{h}^{*} \pi_{h} f\right\|_{\mathcal{D}\left(A_{h}^{-\frac{1}{2}}\right)} \leq C h^{\frac{\theta}{\tau}}\|f\|_{V},
$$

for some $C>0$.

Proof. As in Lemma 7.1, we set

$$
v_{h}=\left(1+h^{\theta}\right)\left(I+h^{\theta} A_{h}^{\frac{l}{2}}\right)^{-1} \pi_{h} f .
$$

First, we notice that

$$
\left\|B_{h} B_{h}^{*}\left(v_{h}-\pi_{h} f\right)\right\|_{H} \leq C\left\|v_{h}-\pi_{h} f\right\|_{H},
$$

and by Lemma 7.1 we get

$$
\left\|B_{h} B_{h}^{*}\left(v_{h}-\pi_{h} f\right)\right\|_{H} \leq C h^{\frac{\theta}{\tau}}\|f\|_{V} .
$$

Second, by Corollary 7.2, we have

$$
\begin{aligned}
\left\|\left(1+h^{\theta}\right)\left(I+h^{\theta} A_{h}^{\frac{l}{2}}\right)^{-1} B_{h} B_{h}^{*} v_{h}-B_{h} B_{h}^{*} v_{h}\right\|_{\mathcal{D}\left(A_{h}^{-\frac{1}{2}}\right)} & \leq C h^{\frac{\theta}{\tau}}\left\|B_{h} B_{h}^{*} v_{h}\right\|_{H} \\
& \leq C h^{\frac{\theta}{l}}\left(\left\|B_{h} B_{h}^{*}\left(v_{h}-\pi_{h} f\right)\right\|_{H}+\left\|B_{h} B_{h}^{*} \pi_{h} f\right\|_{H}\right) \\
& \leq C h^{\frac{\theta}{l}}\|f\|_{V},
\end{aligned}
$$

where we use the fact that $\left\|\pi_{h} f\right\|_{H} \leq c\left\|\pi_{h} f\right\|_{V} \leq c\|f\|_{V}$. The conclusion follows from the two above estimates.

Theorem 7.5. If $z=(f, g)^{\top} \in \mathcal{D}(A) \times \mathcal{D}(A)$, then

$$
\left\|\left(\tilde{A}_{l, h}\right)^{-1}\left(\pi_{h} f, \pi_{h} g\right)^{\top}-\tilde{A}^{-1}(f, g)^{\top}\right\|_{X} \rightarrow 0 \text { as } h \rightarrow 0 .
$$

Proof. By the definition of $\tilde{A}_{l, h}$ and $\tilde{A}$, we have

$$
\left(u_{h}, v_{h}\right)^{\top}=\left(\tilde{A}_{l, h}\right)^{-1}\left(\pi_{h} f, \pi_{h} g\right)^{\top},
$$


and

$$
(u, v)^{\top}=\tilde{A}^{-1}(f, g)^{\top},
$$

if and only if

$$
\left\{\begin{array}{l}
v_{h} \quad=\left(1+h^{\theta}\right)\left(I+h^{\theta} A_{h}^{\frac{l}{2}}\right)^{-1} \pi_{h} f \\
-A_{h} u_{h}=\left(1+h^{\theta}\right)\left(I+h^{\theta} A_{h}^{\frac{l}{2}}\right)^{-1}\left(h^{\theta} A_{h}^{1+\frac{l}{2}}+B_{h} B_{h}^{*}\right) v_{h}+\left(1+h^{\theta}\right)\left(I+h^{\theta} A_{h}^{\frac{l}{2}}\right)^{-1} \pi_{h} g,
\end{array}\right.
$$

and

$$
\left\{\begin{array}{l}
v=f \\
-A u=B B^{*} v+g .
\end{array}\right.
$$

Therefore, we can write

$$
-A_{h} u_{h}=\pi_{h} g+B_{h} B_{h}^{*} \pi_{h} f+r_{h}
$$

where $r_{h} \in V_{h}$ is given by

$$
\begin{aligned}
r_{h}= & \left(1+h^{\theta}\right)\left(I+h^{\theta} A_{h}^{\frac{l}{2}}\right)^{-1} \pi_{h} g-\pi_{h} g \\
& +\left(1+h^{\theta}\right) h^{\theta}\left(I+h^{\theta} A_{h}^{\frac{l}{2}}\right)^{-1} A_{h}^{1+\frac{l}{2}} v_{h} \\
& +\left(1+h^{\theta}\right)\left(I+h^{\theta} A_{h}^{\frac{l}{2}}\right)^{-1} B_{h} B_{h}^{*} v_{h}-B_{h} B_{h}^{*} \pi_{h} f .
\end{aligned}
$$

By the previous Lemmas, $r_{h}$ satisfies

$$
\left\|r_{h}\right\|_{\mathcal{D}\left(A_{h}^{-\frac{1}{2}}\right)} \leq C h^{\frac{\theta}{l}}\left\|(f, g)^{\top}\right\|_{\mathcal{D}(A) \times V} .
$$

Therefore, $u_{h} \in V_{h}$ can be seen as the unique solution of

$$
a\left(u_{h}, w_{h}\right)=-\left(\pi_{h} g, w_{h}\right)-\left(B_{h} B_{h}^{*} \pi_{h} f, w_{h}\right)-\left\langle r_{h} ; w_{h}\right\rangle \quad \forall w_{h} \in V_{h},
$$

where $\langle;\rangle$ denotes the dual product in $D\left(A_{h}^{-\frac{1}{2}}\right)$. Since $u \in V$ is solution of

$$
a(u, w)=-(g, w)-\left(B B^{*} f, w\right) \quad \forall w \in V,
$$

we get (recalling that $V_{h} \subset V$ )

$$
a\left(u, w_{h}\right)=-\left(g, w_{h}\right)-\left(B B^{*} f, w_{h}\right) \quad \forall w_{h} \in V_{h} .
$$

Hence, taking the difference of this identity with (7.6), we obtain

$$
a\left(u-u_{h}, w_{h}\right)=\left(\pi_{h} g-g, w_{h}\right)+\left(B^{*}\left(\pi_{h} f-f\right), B^{*} w_{h}\right)_{U}+\left\langle r_{h} ; w_{h}\right\rangle \quad \forall w_{h} \in V_{h} .
$$

Consequently, taking $w_{h}=\pi_{h} u-u_{h}$, we get

$$
\begin{aligned}
a\left(u-u_{h}, u-u_{h}\right)= & a\left(u-u_{h}, u-\pi_{h} u\right)+a\left(u-u_{h}, \pi_{h} u-u_{h}\right) \\
= & a\left(u-u_{h}, u-\pi_{h} u\right)+\left(\pi_{h} g-g, \pi_{h} u-u_{h}\right) \\
& +\left(B^{*}\left(\pi_{h} f-f\right), B^{*}\left(\pi_{h} u-u_{h}\right)\right)_{U}+\left\langle r_{h} ; \pi_{h} u-u_{h}\right\rangle .
\end{aligned}
$$

Hence, by Cauchy-Schwarz's inequality and the boundedness of $B^{*}$, we obtain

$$
\begin{aligned}
\left\|u-u_{h}\right\|_{V}^{2} & =a\left(u-u_{h}, u-u_{h}\right) \\
& \leq\left\|u-u_{h}\right\|_{V}\left\|u-\pi_{h} u\right\|_{V}+C\left(\left\|\pi_{h} g-g\right\|_{H}+\left\|\pi_{h} f-f\right\|_{H}+\left\|r_{h}\right\|_{\mathcal{D}\left(A_{h}^{-\frac{1}{2}}\right)}\right)\left\|\pi_{h} u-u_{h}\right\|_{V} .
\end{aligned}
$$


Now, using the triangle inequality, we get

$$
\begin{aligned}
\left\|u-u_{h}\right\|_{V}^{2} \leq & C\left(\left(\left\|u-\pi_{h} u\right\|_{V}+\left\|\pi_{h} g-g\right\|_{H}+\left\|\pi_{h} f-f\right\|_{H}+\left\|r_{h}\right\|_{\mathcal{D}\left(A_{h}^{-\frac{1}{2}}\right)}\right)\left\|u-u_{h}\right\|_{V}\right. \\
& \left.+\left(\left\|\pi_{h} g-g\right\|_{H}+\left\|\pi_{h} f-f\right\|_{H}+\left\|r_{h}\right\|_{\mathcal{D}\left(A_{h}^{-\frac{1}{2}}\right)}\right)\left\|u-\pi_{h} u\right\|_{V}\right) .
\end{aligned}
$$

Hence, by Young's inequality, we arrive at

$$
\begin{aligned}
\left\|u-u_{h}\right\|_{V}^{2} \leq & C\left(\left\|u-\pi_{h} u\right\|_{V}^{2}+\left\|\pi_{h} g-g\right\|_{H}^{2}+\left\|\pi_{h} f-f\right\|_{H}^{2}+\left\|r_{h}\right\|_{\mathcal{D}\left(A_{h}^{-\frac{1}{2}}\right)}^{2}\right. \\
& \left.+\left(\left\|\pi_{h} g-g\right\|_{H}+\left\|\pi_{h} f-f\right\|_{H}+\left\|r_{h}\right\|_{\mathcal{D}\left(A_{h}^{-\frac{1}{2}}\right)}\right)\left\|u-\pi_{h} u\right\|_{V}\right) .
\end{aligned}
$$

The estimates (1.5), (1.6), and (7.5) then yield

$$
\begin{aligned}
\left\|u-u_{h}\right\|_{V}^{2} \leq & C\left(h^{2 \theta}\|u\|_{\mathcal{D}(A)}^{2}+h^{4 \theta}\|f\|_{\mathcal{D}(A)}^{2}+h^{4 \theta}\|g\|_{\mathcal{D}(A)}^{2}+h^{\frac{2 \theta}{l}}\left\|(f, g)^{\top}\right\|_{\mathcal{D}(A) \times V}^{2}\right. \\
& \left.+\left(h^{2 \theta}\|f\|_{\mathcal{D}(A)}+h^{2 \theta}\|g\|_{\mathcal{D}(A)}+h^{\frac{\theta}{l}}\left\|(f, g)^{\top}\right\|_{\mathcal{D}(A) \times V}\right) h^{\theta}\|u\|_{\mathcal{D}(A)}\right) .
\end{aligned}
$$

For $v-v_{h}$, we notice that

$$
v-v_{h}=f-\left(1+h^{\theta}\right)\left(I+h^{\theta} A_{h}^{\frac{l}{2}}\right)^{-1} \pi_{h} f=f-\pi_{h} f+\pi_{h} f-\left(1+h^{\theta}\right)\left(I+h^{\theta} A_{h}^{\frac{l}{2}}\right)^{-1} \pi_{h} f,
$$

and we conclude that it tends to zero in $H$ due to the estimate (1.5) and Lemma 7.1.

Corollary 7.6. If $z=(f, g)^{\top} \in V \times H$, recalling that $j_{h}$ is the projection from $H$ into $V_{h}$, we have

$$
\left\|\left(\tilde{A}_{l, h}\right)^{-1}\left(\pi_{h} f, j_{h} g\right)^{\top}-\tilde{A}^{-1}(f, g)^{\top}\right\|_{X} \rightarrow 0 \text { as } h \rightarrow 0 .
$$

Proof. First for $z=(f, g)^{\top} \in \mathcal{D}(A) \times \mathcal{D}(A)$, then

$$
\begin{aligned}
\left\|\left(\tilde{A}_{l, h}\right)^{-1}\left(\pi_{h} f, j_{h} g\right)^{\top}-\tilde{A}^{-1}(f, g)^{\top}\right\|_{X} \leq & \left\|\left(\tilde{A}_{l, h}\right)^{-1}\left(\pi_{h} f, \pi_{h} g\right)^{\top}-\tilde{A}^{-1}(f, g)^{\top}\right\|_{X} \\
& +\left\|\left(\tilde{A}_{l, h}\right)^{-1}\left(0, j_{h} g-\pi_{h} g\right)^{\top}\right\|_{X} .
\end{aligned}
$$

The first term of this right-hand side tends to zero as $h$ goes to zero by the previous Theorem. On the other hand for the second term, as $\tilde{A}_{l, h}$ satisfies (3.2) (see Sect. 6), there exists $C>0$ (independent of $h$ ) such that for all $h<h^{*}$

$$
\left\|\left(\tilde{A}_{l, h}\right)^{-1}\left(0, j_{h} g-\pi_{h} g\right)^{\top}\right\|_{X} \leq C\left\|j_{h} g-\pi_{h} g\right\|_{H} .
$$

Hence, by the triangle inequality and the property $\left\|g-j_{h} g\right\|_{H} \leq\left\|g-\pi_{h} g\right\|_{H}$ (as $j_{h}$ in the projection on $V_{h}$ in $H)$, we get

$$
\left\|\left(\tilde{A}_{l, h}\right)^{-1}\left(0, j_{h} g-\pi_{h} g\right)^{\top}\right\|_{X} \leq 2 C\left\|g-\pi_{h} g\right\|_{H} .
$$

By the estimate (1.6), we then conclude that this second term tends also to zero as $h$ goes to zero.

If $z=(f, g)^{\top}$ is only in $V \times H$, then for an arbitrary $\varepsilon>0$, we use the density of $\mathcal{D}(A) \times \mathcal{D}(A)$ into $V \times H$ to get $(F, G)^{\top} \in \mathcal{D}(A) \times \mathcal{D}(A)$ such that

$$
\left\|(f, g)^{\top}-(F, G)^{\top}\right\|_{X} \leq \varepsilon
$$

Now, by the triangle inequality, we have

$$
\begin{aligned}
\left\|\left(\tilde{A}_{l, h}\right)^{-1}\left(\pi_{h} f, j_{h} g\right)^{\top}-\tilde{A}^{-1}(f, g)^{\top}\right\|_{X} \leq & \left\|\left(\tilde{A}_{l, h}\right)^{-1}\left(\pi_{h}(f-F), j_{h}(g-G)\right)^{\top}\right\|_{X} \\
& +\left\|\tilde{A}^{-1}(f-F, g-G)^{\top}\right\|_{X} \\
& +\left\|\left(\tilde{A}_{l, h}\right)^{-1}\left(\pi_{h} F, j_{h} G\right)^{\top}-\tilde{A}^{-1}(F, G)^{\top}\right\|_{X} .
\end{aligned}
$$


By the first step, there exists $h_{\varepsilon}$ small enough such that

$$
\left\|\left(\tilde{A}_{l, h}\right)^{-1}\left(\pi_{h} F, j_{h} G\right)^{\top}-\tilde{A}^{-1}(F, G)^{\top}\right\|_{X} \leq \varepsilon, \forall 0<h<h_{\varepsilon} .
$$

For the second term, by the boundedness of $\tilde{A}^{-1}$, we may write

$$
\left\|\tilde{A}^{-1}(f-F, g-G)^{\top}\right\|_{X} \leq C\left\|(f-F, g-G)^{\top}\right\|_{X} \leq C \varepsilon .
$$

Finally for the first term, using the property (3.2) and the fact that $\pi_{h}$ (resp. $j_{h}$ ) is a projection from $V$ (resp. from $H$ ) into $V_{h}$, we get for all $h<h^{*}$

$$
\left\|\left(\tilde{A}_{l, h}\right)^{-1}\left(\pi_{h}(f-F), j_{h}(g-G)\right)^{\top}\right\|_{X} \leq C\left\|\left(\pi_{h}(f-F), j_{h}(g-G)\right)^{\top}\right\|_{X} \leq C\left\|(f-F, g-G)^{\top}\right\|_{X} \leq C \varepsilon .
$$

All together we have obtained that

$$
\left\|\left(\tilde{A}_{l, h}\right)^{-1}\left(\pi_{h} f, j_{h} g\right)^{\top}-\tilde{A}^{-1}(f, g)^{\top}\right\|_{X} \leq(1+2 C) \varepsilon, \quad \forall 0<h<\min \left\{h_{\varepsilon}, h^{*}\right\} .
$$

This proves the result.

We are now ready to state the convergence result.

Theorem 7.7. If $\left(\omega_{0}, \omega_{1}\right)^{\top} \in V \times H$, then

$$
\left\|T_{l, h}(t)\left(\pi_{h} \omega_{0}, j_{h} \omega_{1}\right)^{\top}-T(t)\left(\omega_{0}, \omega_{1}\right)^{\top}\right\|_{X} \rightarrow 0 \text { as } h \rightarrow 0 .
$$

Proof. We use Theorem 2.1 of [23] with $X=Z=V \times H, X_{n}=V_{h} \times V_{h}$, and $P_{n}: X \rightarrow X_{n}$ defined by

$$
P_{n}(f, g)^{\top}=\left(\pi_{h} f, j_{h} g\right)^{\top}, \forall(f, g)^{\top} \in X,
$$

and $E_{n}=P_{n}^{*}$ that is here the canonical injection of $V_{h} \times V_{h}$ into $V \times H$. The Assumptions (A1) and (A3) of [23] are trivially satisfied, while the assumption (A2) is a consequence of $(1.5),(1.6)$ and the density of $\mathcal{D}(A) \times \mathcal{D}(A)$ into $V \times H$.

Since Corollary 7.6 shows that point (a) of Theorem 2.1 of [23] holds, we conclude that point (b) of this Theorem, namely (7.8), holds.

\section{EXAMPLES}

\subsection{Two coupled wave equations}

We consider the following system

$$
\begin{cases}u_{t t}(x, t)-u_{x x}(x, t)+\alpha y(x, t)+\beta(x) u_{t}(x, t)=0 & \text { in }(0,1) \times \mathbb{R}_{+}, \\ y_{t t}(x, t)-y_{x x}(x, t)+\alpha u(x, t)+\gamma(x) y_{t}(x, t)=0 & \text { in }(0,1) \times \mathbb{R}_{+}, \\ u(0, t)=u(1, t)=y(0, t)=y(1, t)=0 & \forall t>0, \\ u(\cdot, 0)=u_{0}, u_{t}(\cdot, 0)=u_{1}, y(\cdot, 0)=y_{0}, y_{t}(\cdot, 0)=y_{1} & \text { in }(0,1),\end{cases}
$$

when $\alpha \in \mathbb{R}$ such that $\alpha>0$ is small enough (see below), $\beta($.$) and \gamma($.$) are two non-negative bounded functions$ such that $\beta(x) \geq \beta>0$ for $x \in I_{\beta} \subseteq(0,1)$ and $\gamma(x) \geq \gamma>0$ for $x \in I_{\gamma} \subseteq(0,1)$ where $I_{\beta}$ and $I_{\gamma}$ are two open sets such that their measures do not vanish simultaneously. Hence, (8.1) is written in the form (1.1) with the following choices: Take $H=L^{2}(0,1)^{2}$, the operator $B$ as follows:

$$
B \omega=\sqrt{\beta(.)}\left(\begin{array}{l}
u \\
0
\end{array}\right)+\sqrt{\gamma(\cdot)}\left(\begin{array}{l}
0 \\
y
\end{array}\right)
$$


when $\omega=\left(\begin{array}{l}u \\ y\end{array}\right)$, which is a bounded operator from $H$ into itself $(i . e . U=H)$ and the operator $A$ defined by

$$
\mathcal{D}(A)=V \cap H^{2}(0,1)^{2},
$$

when $V=H_{0}^{1}(0,1)^{2}$ and

$$
A \omega=\left(\begin{array}{l}
-u_{x x}+\alpha y \\
-y_{x x}+\alpha u
\end{array}\right)
$$

If $\alpha$ is small enough, namely if $\alpha<\pi^{2}$, this operator $A$ is a positive selfadjoint operator in $H$, since it is the Friedrichs extension of the triple $(H, V, a)$, where the sesquilinear form $a$ is defined by

$$
a\left(\omega, \omega^{*}\right)=\int_{0}^{1}\left(u_{x}\left(\overline{u^{*}}\right)_{x}+y_{x}\left(\overline{y^{*}}\right)_{x}+\alpha y \overline{u^{*}}+\alpha u \overline{y^{*}}\right) \mathrm{d} x, \forall \omega=\left(\begin{array}{l}
u \\
y
\end{array}\right), \omega^{*}=\left(\begin{array}{l}
u^{*} \\
y^{*}
\end{array}\right) \in V .
$$

Indeed $a$ is clearly a continuous symmetric sesquilinear form on $V$ and is coercive if $\alpha<\pi^{2}$ due to Poincaré's inequality

$$
\int_{0}^{1}\left|u_{x}\right|^{2} \mathrm{~d} x \geq \pi^{2} \int_{0}^{1}|u|^{2} \mathrm{~d} x, \quad \forall u \in H_{0}^{1}(0,1) .
$$

Furthermore, $A$ has a compact resolvent since $\mathcal{D}(A)$ is compactly embedded into $H$.

Let us now check that the generalized gap condition (1.7) and the Assumptions (1.10) or (1.13) are satisfied for our system (8.1). We start by the determination of the spectrum of the operator $A$. Hence we are looking for $\omega=(u, y)^{\top} \in V \cap H^{2}(0,1)^{2}$ different from 0 and $\lambda^{2}>0$ solution of

$$
\begin{aligned}
& -u_{x x}+\alpha y=\lambda^{2} u \text { in }(0,1), \\
& -y_{x x}+\alpha u=\lambda^{2} y \text { in }(0,1) .
\end{aligned}
$$

If such a pair exists, we can set

$$
s=\frac{u+y}{2}, \quad d=\frac{u-y}{2},
$$

and notice that $s$ and $d$ belong to $H_{0}^{1}(0,1) \cap H^{2}(0,1)$ and are solution of

$$
\begin{aligned}
& -s_{x x}+\alpha s=\lambda^{2} s \text { in }(0,1), \\
& -d_{x x}-\alpha d=\lambda^{2} d \text { in }(0,1) .
\end{aligned}
$$

Hence $s($ resp. $d)$ is an eigenvector of the Laplace operator $-\frac{\mathrm{d}}{\mathrm{d} x^{2}}$ with Dirichlet boundary condition of eigenvalue $\lambda^{2}-\alpha$ (resp. $\left.\lambda^{2}+\alpha\right)$. A first choice is then to have for all $k \in \mathbb{N}^{*}: \lambda^{2}=k^{2} \pi^{2}+\alpha, s=\sin (k \pi \cdot)$ and $d=0$. Coming back to $(u, y)$, we find (since $u=s+d$ and $y=s-d$ ) a sequence of eigenvalues $\lambda_{+, k}^{2}=k^{2} \pi^{2}+\alpha$ of associated eigenvector

$$
\omega_{+, k}=(\sin (k \pi \cdot), \sin (k \pi \cdot)) .
$$

Note that each eigenvalue is simple and that $\omega_{+, k}$ is of norm 1 in $H$.

A second choice is to take for all $k \in \mathbb{N}^{*}: \lambda^{2}=k^{2} \pi^{2}-\alpha$ (which is meaningful since $\alpha<\pi^{2}$ ), $s=0$ and $d=\sin (k \pi \cdot)$. Again coming back to $(u, y)$, we find a sequence of eigenvalues $\lambda_{-, k}^{2}=k^{2} \pi^{2}-\alpha$ of associated eigenvector

$$
\omega_{-, k}=(\sin (k \pi \cdot),-\sin (k \pi \cdot)) .
$$

As before each eigenvalue is simple and $\omega_{-, k}$ is of norm 1 in $H$.

Now we remark that the sequence $\left\{\omega_{+, k}\right\}_{k \in \mathbb{N}^{*}} \cup\left\{\omega_{-, k}\right\}_{k \in \mathbb{N}^{*}}$ is an orthonormal basis of $H$ (because $\omega_{+, k}+$ $\omega_{-, k}=2(\sin (k \pi \cdot), 0)$ and $\left.\omega_{+, k}-\omega_{-, k}=2(0, \sin (k \pi \cdot))\right)$ and therefore we have found all possible eigenvectors of $A$. We have then shown that the spectrum of $A$ is given by

$$
\operatorname{Sp}(A)=\left\{\lambda_{+, k}^{2}\right\}_{k \in \mathbb{N}^{*}} \cup\left\{\lambda_{-, k}^{2}\right\}_{k \in \mathbb{N}^{*}}
$$

and that each eigenvalue is simple (because the assumption $\alpha<\pi^{2}$ implies that $\left.k^{2} \pi^{2}+\alpha<(k+1)^{2} \pi^{2}-\alpha\right)$. 
We now need to estimate the distance between the consecutive eigenvalues of $A^{1 / 2}$. We have two different cases to consider:

1. For all $k \in \mathbb{N}^{*}$, we need to look at the distance between $\lambda_{+, k}$ and $\lambda_{-, k}$. Since

$$
\lambda_{+, k}-\lambda_{-, k}=\sqrt{k^{2} \pi^{2}+\alpha}-\sqrt{k^{2} \pi^{2}-\alpha}=\frac{2 \alpha}{\sqrt{k^{2} \pi^{2}+\alpha}+\sqrt{k^{2} \pi^{2}-\alpha}},
$$

we see that this distance goes to zero as $k$ goes to infinity.

2. For all $k \in \mathbb{N}^{*}$, we look at the distance between $\lambda_{+, k}$ and $\lambda_{-, k+1}$. Here we have

$$
\lambda_{-, k+1}-\lambda_{+, k}=\sqrt{(k+1)^{2} \pi^{2}-\alpha}-\sqrt{k^{2} \pi^{2}+\alpha}=\frac{2 k \pi^{2}+\pi^{2}-2 \alpha}{\sqrt{(k+1)^{2} \pi^{2}-\alpha}+\sqrt{k^{2} \pi^{2}+\alpha}},
$$

which tends to $\pi$ as $k$ goes to infinity.

This shows that the generalized gap condition (1.7) is satisfied with $M=2$. With the terminology of Section 1 , we see that $A_{1}=\emptyset$ and $A_{2}=\mathbb{N}^{*}$.

In order to check (1.10) or (1.13), for all $k \in \mathbb{N}^{*}$, we set

$$
\alpha_{k}=\lambda_{+, k}-\lambda_{-, k},
$$

that behaves like $k^{-1}$ or equivalently like $\lambda_{-, k}^{-1}$. We further need to use the matrix (see Lem. 1.1)

$$
B_{k}^{-1}=\left(\begin{array}{cc}
1 & 1 \\
0 & \alpha_{k}
\end{array}\right)
$$

as well as the matrix $\Phi_{k}$ which here takes the form

$$
\Phi_{k}=\left(\begin{array}{cc}
B^{*} \omega_{-, k} & 0 \\
0 & B^{*} \omega_{+, k}
\end{array}\right) .
$$

Hence for all $C=\left(c_{1}, c_{2}\right)^{\top} \in \mathbb{R}^{2}$, we have

$$
B_{k}^{-1} \Phi_{k} C=\left(\begin{array}{c}
c_{1} B^{*} \omega_{-, k}+c_{2} B^{*} \omega_{+, k} \\
\alpha_{k} c_{2} B^{*} \omega_{+, k}
\end{array}\right)
$$

and consequently

$$
\begin{aligned}
\left\|B_{k}^{-1} \Phi_{k} C\right\|_{U, 2}^{2}= & \left\|c_{1} B^{*} \omega_{-, k}+c_{2} B^{*} \omega_{+, k}\right\|_{2}^{2}+\left|\alpha_{k}\right|^{2}\left|c_{2}\right|^{2}\left\|B^{*} \omega_{+, k}\right\|_{2}^{2} \\
= & \left|c_{1}+c_{2}\right|^{2} \int_{0}^{1} \beta(x) \sin ^{2}(k \pi x) \mathrm{d} x+\left|c_{2}-c_{1}\right|^{2} \int_{0}^{1} \gamma(x) \sin ^{2}(k \pi x) \mathrm{d} x \\
& +\left|\alpha_{k}\right|^{2}\left|c_{2}\right|^{2} \int_{0}^{1}(\beta(x)+\gamma(x)) \sin ^{2}(k \pi x) \mathrm{d} x .
\end{aligned}
$$

We have two different cases to consider:

First case. $I_{\beta} \neq \emptyset$ and $I_{\gamma} \neq \emptyset$.

In this case, we have

$$
\begin{aligned}
\left\|B_{k}^{-1} \Phi_{k} C\right\|_{U, 2}^{2} & \geq \min \{\beta, \gamma\} \min \left\{\int_{I_{\beta}} \sin ^{2}(k \pi x) \mathrm{d} x, \int_{I_{\gamma}} \sin ^{2}(k \pi x) \mathrm{d} x\right\}\left(\left(c_{1}+c_{2}\right)^{2}+\left(c_{2}-c_{1}\right)^{2}\right) \\
& =2 \min \{\beta, \gamma\} \min \left\{\int_{I_{\beta}} \sin ^{2}(k \pi x) \mathrm{d} x, \int_{I_{\gamma}} \sin ^{2}(k \pi x) \mathrm{d} x\right\}\left(c_{1}^{2}+c_{2}^{2}\right)
\end{aligned}
$$


and hence (1.10) holds since $\min \left\{\int_{I_{\beta}} \sin ^{2}(k \pi x) \mathrm{d} x, \int_{I_{\gamma}} \sin ^{2}(k \pi x) \mathrm{d} x\right\}$ is uniformly bounded from below. Indeed, as $I_{\gamma} \neq \emptyset$, there exists $a \in(0,1)$ and $\epsilon>0$ such that $(a, a+\epsilon) \subset I_{\gamma}$, and therefore

$$
\int_{I_{\gamma}} \sin ^{2}(k \pi x) \mathrm{d} x \geq \frac{\epsilon}{2}+\frac{1}{4 k \pi}(\sin (2 k \pi a)-\sin (2 k \pi(a+\epsilon))) \geq \frac{\epsilon}{2}-\frac{1}{2 k \pi} \geq \frac{\epsilon}{4},
$$

for $k \geq \frac{2}{\epsilon \pi}$. On the other hand, we clearly have

$$
\min _{1 \leq k<\frac{2}{\epsilon \pi}} \int_{I_{\gamma}} \sin ^{2}(k \pi x) \mathrm{d} x>0
$$

which shows that $\int_{I_{\gamma}} \sin ^{2}(k \pi x) \mathrm{d} x$ is uniformly bounded from below.

Second case. $I_{\beta}=\emptyset$ or $I_{\gamma}=\emptyset$ (but not empty together). For instance, suppose that $I_{\beta}=\emptyset$ and $I_{\gamma} \neq \emptyset$. As $\left|\alpha_{k}\right| \sim \lambda_{-, k}^{-1}$, we deduce that

$$
\left\|B_{k}^{-1} \Phi_{k} C\right\|_{U, 2} \geq \alpha_{0} \lambda_{-, k}^{-1}\|C\|_{2},
$$

for a positive constant $\alpha_{0}$, and shows that (1.13) holds with $l=1$.

As stated before, in the first case the system (8.1) is exponentially stable, while in the second case (8.1) is polynomially stable. We refer to Theorem 2.4 of $[3]$ or to $[1,29]$ for the proof of these results.

As approximated space $V_{h}$, we use the standard one based on $P 1$ finite elements. More precisely, for $N \in \mathbb{N}$ and $h=\frac{1}{N+1}$, we define the points $x_{j}=j h, j=0,1, \ldots, N+1$. The space $V_{h}$ is the linear span of the family of hat functions $\left(e_{i}, e_{j}\right)_{i, j \in\{1, \ldots, N\}}$ such that

$$
e_{j}(x)=\left[1-\frac{\left|x-x_{j}\right|}{h}\right]^{+}, \text {for } j=1, \ldots, N
$$

Then, we define the operators $A_{h}$ and $B_{h}$ by (1.2) and (1.4). It is well-known (see [12]) that the operator $A$ and the space $V_{h}$ satisfy conditions (1.5) and (1.6) with $\theta=1$.

Consequently, in the first case $\left(I_{\beta} \neq \emptyset\right.$ and $\left.I_{\gamma} \neq \emptyset\right)$, we can apply Theorem 1.2 and thus the family of systems (1.9) is uniformly exponentially stable, in the sense that there exist constants $M, \alpha, h^{*}>0$ (independent of $\left.h, u_{0 h}, u_{1 h}, y_{0 h}, y_{1 h}\right)$ such that for all $h \in\left(0, h^{*}\right)$ :

$$
\left\|\dot{\omega}_{h}(t)\right\|^{2}+a\left(\omega_{h}(t), \omega_{h}(t)\right) \leq M \mathrm{e}^{-\alpha t}\left(\left\|\omega_{1 h}\right\|^{2}+a\left(\omega_{0 h}, \omega_{0 h}\right)\right), \forall t \geq 0,
$$

where $\omega_{h}=\left(u_{h}, y_{h}\right)$, and $\omega_{0 h}=\left(u_{0 h}, y_{0 h}\right) \in V_{h}$ (resp. $\left.\omega_{1 h}=\left(u_{1 h}, y_{1 h}\right) \in V_{h}\right)$ is an approximation of $\omega_{0}=$ $\left(u_{0}, y_{0}\right)$ (resp. $\left.\omega_{1}=\left(u_{1}, y_{1}\right)\right)$.

In the second case $\left(I_{\beta}=\emptyset\right.$ and $\left.I_{\gamma} \neq \emptyset\right)$, we can apply Theorem 1.6 with $l=2$ and thus the family of systems (1.14) is uniformly polynomially stable, in the sense that, there exist constants $C, h^{*}>0$ (independent of $\left.h, u_{0 h}, u_{1 h}, y_{0 h}, y_{1 h}\right)$ such that for all $h \in\left(0, h^{*}\right)$ :

$$
\left\|\left(I+h A_{h}\right)^{-1} \dot{\omega}_{h}(t)\right\|^{2}+a\left(\omega_{h}(t), \omega_{h}(t)\right) \leq \frac{C}{\sqrt{t}}\left\|\left(\omega_{0 h}, \omega_{1 h}\right)\right\|_{D\left(\tilde{A}_{2, h}\right)}^{2} \forall t>0,
$$

where $\tilde{A}_{2, h}$ is given as in (4.1) with $l=2, \theta=1$, and the the graph norm $\|\cdot\|_{D\left(\tilde{A}_{2, h}\right)}$ is defined by

$$
\left\|\left(\omega_{0 h}, \omega_{1 h}\right)\right\|_{D\left(\tilde{A}_{2, h}\right)}^{2}=\left\|\left(\omega_{0 h}, \omega_{1 h}\right)\right\|_{X_{h}}^{2}+\left\|\tilde{A}_{2, h}\left(\omega_{0 h}, \omega_{1 h}\right)\right\|_{X_{h}}^{2} .
$$




\subsection{Two boundary coupled wave equations}

We consider the following system

$$
\begin{cases}u_{t t}-u_{x x}=0 & \text { in }(0,1) \times \mathbb{R}_{+}, \\ y_{t t}-y_{x x}+\beta y_{t}=0 & \text { in }(0,1) \times \mathbb{R}_{+}, \\ u(0, t)=y(0, t)=0 & \forall t>0, \\ y_{x}(1, t)=\alpha u(1, t) & \forall t>0, \\ u_{x}(1, t)=\alpha y(1, t) & \forall t>0, \\ u(\cdot, 0)=0, u_{t}(\cdot, 0)=u_{1}, y(\cdot, 0)=0, y_{t}(\cdot, 0)=y_{1} & \text { in }(0,1),\end{cases}
$$

when $\alpha, \beta \in \mathbb{R}$ with $\beta>0$ and $\alpha>0$ small enough (see below). Hence it is written in the form (1.1) with the following choices: take $H=L^{2}(0,1)^{2}$, the operator $B$ as follows:

$$
B \omega=\sqrt{\beta}\left(\begin{array}{l}
0 \\
y
\end{array}\right)
$$

when $\omega=\left(\begin{array}{l}u \\ y\end{array}\right)$, which is a bounded operator from $H$ into itself $(i . e . U=H)$ and the operator $A$ defined by

$$
\mathcal{D}(A)=\left\{(u, y) \in V \cap H^{2}(0,1)^{2}: y_{x}(1)=\alpha u(1) ; u_{x}(1)=\alpha y(1)\right\}
$$

when $V=\left\{\omega \in H^{1}(0,1)^{2}: \omega(0)=0\right\}$ and

$$
A \omega=\left(\begin{array}{l}
-u_{x x} \\
-y_{x x}
\end{array}\right)
$$

If $\alpha$ is small enough, namely if $\alpha<1$, this operator $A$ is a positive selfadjoint operator in $H$, since it is the Friedrichs extension of the triple $(H, V, a)$, where the sesquilinear form $a$ is defined by

$$
a\left(\omega, \omega^{*}\right)=\int_{0}^{1}\left(u_{x}\left(\overline{u^{*}}\right)_{x}+y_{x}\left(\overline{y^{*}}\right)_{x}\right) \mathrm{d} x-\alpha u(1) \overline{y^{*}}(1)-\alpha \overline{u^{*}}(1) y(1), \quad \forall \omega=\left(\begin{array}{l}
u \\
y
\end{array}\right), \omega^{*}=\left(\begin{array}{l}
u^{*} \\
y^{*}
\end{array}\right) \in V .
$$

Indeed $a$ is clearly a continuous symmetric sesquilinear form on $V$ and is coercive if $\alpha<1$ due to the trace theorem

$$
u(1)^{2} \leq \int_{0}^{1}\left|u_{x}\right|^{2} \mathrm{~d} x, \quad \forall u \in V .
$$

In addition to that, the operator $A$ admits a compact resolvent as $\mathcal{D}(A)$ is compactly embedded in $H$.

Let us now check that the generalized gap condition (1.7) and the Assumption (1.13) are satisfied for our system (8.4). We start by the determination of the spectrum of the operator A. Hence we are looking for $\omega=(u, y)^{\top} \in \mathcal{D}(A)$ different from 0 and $\lambda^{2}>0$ solution of

$$
\begin{aligned}
& -u_{x x}=\lambda^{2} u \text { in }(0,1), \\
& -y_{x x}=\lambda^{2} y \text { in }(0,1) .
\end{aligned}
$$

Then

$$
\begin{gathered}
u(x)=a \sin (\lambda x) \text { in }(0,1), \\
y(x)=b \sin (\lambda x) \text { in }(0,1) .
\end{gathered}
$$

The coupling condition in (8.4) gives

$$
\left\{\begin{array}{l}
a \lambda \cos \lambda=\alpha b \sin \lambda \\
b \lambda \cos \lambda=\alpha a \sin \lambda
\end{array}\right.
$$


Since it is not possible to have $\sin \lambda=0$ (otherwise $a=b=0$ ), we obtain

$$
a=\frac{b \lambda \cos \lambda}{\alpha \sin \lambda}
$$

and then

$$
\tan \lambda= \pm \frac{\lambda}{\alpha}
$$

because $b \neq 0$ (otherwise $u=y=0$ ).

We then have two sequences of eigenvalues defined by

$$
\lambda_{-, k}=\frac{\pi}{2}+k \pi-\epsilon_{-, k}
$$

with $\lim _{k \rightarrow+\infty} \epsilon_{-, k}=0$ and $\epsilon_{-, k}>0$ for all $k \in \mathbb{N}$, and

$$
\lambda_{+, k}=\frac{\pi}{2}+k \pi+\epsilon_{+, k}
$$

with $\lim _{k \rightarrow+\infty} \epsilon_{+, k}=0$ and $\epsilon_{+, k}>0$ for all $k \in \mathbb{N}$. Moreover as $\lambda_{-, k}$ and $\lambda_{+, k}$ satisfies (8.6), we can verify that

$$
\epsilon_{-, k}=\arctan \left(\frac{\alpha}{\lambda_{-, k}}\right) \text { and } \epsilon_{+, k}=\arctan \left(\frac{\alpha}{\lambda_{+, k}}\right) .
$$

By (8.5) and (8.6), the eigenvector associated with the eigenvalue $\lambda_{+, k}$ is given by

$$
\omega_{+, k}=b_{+, k} \sin \left(\lambda_{+, k} \cdot\right)(-1,1)^{T},
$$

and the eigenvector associated with the eigenvalue $\lambda_{-, k}$ is given by

$$
\omega_{-, k}=b_{-, k} \sin \left(\lambda_{-, k} \cdot\right)(1,1)^{T},
$$

where $b_{+, k}, b_{-, k}$ are chosen to normalize the eigenvectors.

Since we have found all possible eigenvectors of $A$, we have shown that the spectrum of $A$ is given by

$$
\operatorname{Sp}(A)=\left\{\lambda_{+, k}^{2}\right\}_{k \in \mathbb{N}^{*}} \cup\left\{\lambda_{-, k}^{2}\right\}_{k \in \mathbb{N}^{*}}
$$

and that each eigenvalue is simple.

We again need to estimate the distance between the consecutive eigenvalues of $A^{1 / 2}$ and as before we consider two different cases:

1. For all $k \in \mathbb{N}^{*}$, we need to look at the distance between $\lambda_{+, k}$ and $\lambda_{-, k}$. Since

$$
\lambda_{+, k}-\lambda_{-, k}=\epsilon_{+, k}+\epsilon_{-, k}=\arctan \left(\frac{\alpha}{\lambda_{+, k}}\right)+\arctan \left(\frac{\alpha}{\lambda_{-, k}}\right),
$$

we see that this distance goes to zero as $k$ goes to infinity.

2. For all $k \in \mathbb{N}^{*}$, we look at the distance between $\lambda_{+, k}$ and $\lambda_{-, k+1}$. Here we have

$$
\lambda_{-, k+1}-\lambda_{+, k}=\pi-\left(\epsilon_{+, k}+\epsilon_{-, k+1}\right),
$$

which tends to $\pi$ as $k$ goes to infinity.

This shows that the generalized gap condition (1.7) is satisfied with $M=2$.

In order to check (1.13), for all $k \in \mathbb{N}^{*}$, we set

$$
\alpha_{k}=\lambda_{+, k}-\lambda_{-, k}
$$


that behaves like $k^{-1}$ or equivalently like $\lambda_{-, k}^{-1}$. As in the previous subsection for all $C=\left(c_{1}, c_{2}\right)^{\top} \in \mathbb{R}^{2}$, we have

$$
B_{k}^{-1} \Phi_{k} C=\left(\begin{array}{c}
c_{1} B^{*} \omega_{-, k}+c_{2} B^{*} \omega_{+, k} \\
\alpha_{k} c_{2} B^{*} \omega_{+, k}
\end{array}\right)
$$

and consequently

$$
\begin{aligned}
\left\|B_{k}^{-1} \Phi_{k} C\right\|_{U, 2}^{2}= & \left\|c_{1} B^{*} \omega_{-, k}+c_{2} B^{*} \omega_{+, k}\right\|_{H}^{2}+\left|\alpha_{k}\right|^{2}\left|c_{2}\right|^{2}\left\|B^{*} \omega_{+, k}\right\|_{H}^{2} \\
= & \beta \int_{0}^{1}\left(b_{-, k} c_{1} \sin \left(\lambda_{-, k} x\right)+b_{+, k} c_{2} \sin \left(\lambda_{+, k} x\right)\right)^{2} \mathrm{~d} x \\
& +\beta\left|\alpha_{k}\right|^{2}\left|c_{2}\right|^{2} b_{+, k}^{2} \int_{0}^{1} \sin ^{2}\left(\lambda_{+, k} x\right) \mathrm{d} x .
\end{aligned}
$$

By using Young's inequality with $\epsilon>0$ and the fact that the eigenvectors are normalized (by the choice of $b_{ \pm, k}$ ), we obtain

$$
\begin{aligned}
\left\|B_{k}^{-1} \Phi_{k} C\right\|_{U, 2}^{2} \geq & \beta\left(1-\frac{1}{\epsilon}\right) c_{1}^{2} b_{-, k}^{2} \int_{0}^{1} \sin ^{2}\left(\lambda_{-, k} x\right) \mathrm{d} x+\beta(1-\epsilon) c_{2}^{2} b_{+, k}^{2} \int_{0}^{1} \sin ^{2}\left(\lambda_{+, k} x\right) \mathrm{d} x \\
& +\beta\left|\alpha_{k}\right|^{2}\left|c_{2}\right|^{2} b_{+, k}^{2} \int_{0}^{1} \sin ^{2}\left(\lambda_{+, k} x\right) \mathrm{d} x \\
= & \frac{\beta}{2}\left(\left(1-\frac{1}{\epsilon}\right) c_{1}^{2}+\left(1+\alpha_{k}^{2}-\epsilon\right) c_{2}^{2}\right) .
\end{aligned}
$$

We then take $\epsilon=1+\alpha_{k}^{2} / 2$, which implies

$$
1+\alpha_{k}^{2}-\epsilon=\frac{\alpha_{k}^{2}}{2} \quad \text { and } \quad 1-\frac{1}{\epsilon}>\frac{\alpha_{k}^{2}}{4}
$$

(since $\left.\alpha_{k}^{2}<2\right)$. Consequently

$$
\left\|B_{k}^{-1} \Phi_{k} C\right\|_{U, 2}^{2} \geq \frac{\beta}{8} \alpha_{k}^{2}\left(c_{1}^{2}+c_{2}^{2}\right) .
$$

As $\left|\alpha_{k}\right| \sim \lambda_{-, k}^{-1}$, we deduce that

$$
\left\|B_{k}^{-1} \Phi_{k} C\right\|_{U, 2} \geq \alpha_{0} \lambda_{-, k}^{-1}\|C\|_{2},
$$

for a positive constant $\alpha_{0}$, and shows that (1.13) holds with $l=1$.

We construct the space $V_{h}$ like in the previous subsection, i.e. it is the span of $\left(e_{i}, e_{j}\right)_{i, j \in\{1, \ldots, N+1\}}$, that still satisfies (1.5) and (1.6) with $\theta=1$.

Consequently, we can apply Theorem 1.6 with $l=2$ and thus the family of systems (1.14) is uniformly polynomially stable, in the sense that the estimate (8.3) holds.

\subsection{A more general wave type system}

We consider the following more general system: let $\omega=\left(\omega_{1}, \cdots, \omega_{N}\right)^{T}$ be a solution of

$$
\begin{cases}\omega_{t t}-\omega_{x x}+M \omega+B B^{*} \omega_{t}=0 & \text { in }(0,1)^{N} \times \mathbb{R}_{+}, \\ \omega(0, t)=\omega(1, t)=0 & \forall t>0, \\ \omega(\cdot, 0)=\omega^{(0)}, \omega_{t}(\cdot, 0)=\omega^{(1)} & \text { in }(0,1)^{N},\end{cases}
$$

where $M \in \mathcal{M}_{N}(\mathbb{R})$ is symmetric and such that $A_{0}+M$ is positive definite in $H=L^{2}(0,1)^{N}$, when $A_{0}$ is the operator of domain $\mathcal{D}\left(A_{0}\right)=H_{0}^{1}(0,1)^{N} \cap H^{2}(0,1)^{N}$ and such that $A_{0} u=-u_{x x}$, for all $u \in \mathcal{D}\left(A_{0}\right) ; B \in \mathcal{L}(U, H)$, with $U$ a complex Hilbert space. 
Hence it is written in the form (1.1) with the self-adjoint positive operator $A$ defined by $A=A_{0}+M$ and $\mathcal{D}(A)=\mathcal{D}\left(A_{0}\right)=V \cap H^{2}(0,1)^{N}$, when $V=H_{0}^{1}(0,1)^{N}$. We remark that $A$ admits a compact resolvent since $\mathcal{D}(A)$ is compactly embedded into $H$.

As $M$ is symmetric, $M$ can be diagonalized by an orthogonal matrix, i.e. there exist a real orthogonal matrix $O$ and a diagonal matrix $D$ such that $O^{T} M O=D$. We denote by $d_{i}(i=1, \cdots, N)$ the coefficients of the diagonal matrix $D$.

We start by the determination of the spectrum of the operator $A$. Hence we are looking for $\omega \in V \cap H^{2}(0,1)^{N}$ different from 0 and $\lambda^{2}>0$ solution of

$$
-\omega_{x x}+M \omega=\lambda^{2} \omega .
$$

If we denote by $U=O^{T} \omega$, then $U=\left(u_{1}, \cdots, u_{N}\right)^{T}$ satisfies

$$
-U_{x x}+D U=\lambda^{2} U,
$$

which is equivalent to

$$
-\frac{\mathrm{d}^{2}}{\mathrm{~d} x^{2}} u_{i}=\left(\lambda^{2}-d_{i}\right) u_{i}, \quad \text { in }(0,1), \quad \forall i=1, \cdots, N .
$$

Hence there exists $c_{i} \in \mathbb{C}$ such that

$$
u_{i}=\sqrt{2} c_{i} \sin (k \pi .), \quad \lambda_{i, k}^{2}=k^{2} \pi^{2}+d_{i}, \quad i=1, \cdots, N .
$$

Therefore we have found $N$ families of eigenvectors and eigenvalues:

$$
U_{i, k}=\sqrt{2} f_{i} \sin (k \pi .), \quad \lambda_{i, k}^{2}=k^{2} \pi^{2}+d_{i}, \quad i=1, \cdots, N,
$$

where $\left(f_{i}\right)_{i \in\{1, \cdots, N\}}$ is the canonical basis of $\mathbb{C}^{N}$. Coming back to the initial eigenvalue problem, we have $\mathrm{N}$ families of eigenvectors given by

$$
\omega_{i, k}=O U_{i, k}, \quad i=1, \cdots, N,
$$

and the spectrum of $A$ is given by

$$
\operatorname{Sp}(A)=\left\{\lambda_{1, k}^{2}\right\}_{k \in \mathbb{N}^{*}} \cup \cdots \cup\left\{\lambda_{N, k}^{2}\right\}_{k \in \mathbb{N}^{*}} .
$$

For simplicity we now assume that all $d_{i}$ are different and, for instance that

$$
d_{1}<d_{2}<\cdots<d_{N} .
$$

We still estimate the distance between the consecutive eigenvalues of $A^{1 / 2}$ :

1. For all $k \in \mathbb{N}^{*}$, we need to look at the distance between $\lambda_{i, k}$ and $\lambda_{j, k}(i \neq j)$. Since

$$
\lambda_{i, k}-\lambda_{j, k}=\sqrt{k^{2} \pi^{2}+d_{i}}-\sqrt{k^{2} \pi^{2}+d_{j}}=\frac{d_{i}-d_{j}}{\sqrt{k^{2} \pi^{2}+d_{i}}+\sqrt{k^{2} \pi^{2}+d_{j}}},
$$

we see that this distance goes to zero as $k$ goes to infinity.

2. For all $k \in \mathbb{N}^{*}$, we look at the distance between $\lambda_{N, k}$ and $\lambda_{1, k+1}$. Here we have

$$
\lambda_{1, k+1}-\lambda_{N, k}=\sqrt{(k+1)^{2} \pi^{2}+d_{1}}-\sqrt{k^{2} \pi^{2}+d_{N}}=\frac{2 k \pi^{2}+\pi^{2}+d_{1}-d_{N}}{\sqrt{(k+1)^{2} \pi^{2}+d_{1}}+\sqrt{k^{2} \pi^{2}+d_{N}}},
$$

which tends to $\pi$ as $k$ goes to infinity.

This shows that the generalized gap condition (1.7) is satisfied with $M=N$. With the terminology of Section 1, we see that $A_{1}=\cdots=A_{N-1}=\emptyset$ and $A_{N}=\mathbb{N}^{*}$. Hence, for $N>1$, our previous results will allow to obtain stability results for system (8.7). 
If the eigenvalues are simple (a necessary condition is that all $d_{i}$ are different), then in order to verify (1.10) or (1.13), we have to bound from below $\left\|B_{k}^{-1} \Phi_{k} C\right\|_{U, 2}^{2}$ with $C=\left(c_{1}, \cdots, c_{N}\right) \in \mathbb{R}^{N}, B_{k}^{-1}$ defined in Lemma 1.1 and $\Phi_{k}$ given by

$$
\Phi_{k}=\left(\begin{array}{ccc}
B^{*} \omega_{1, k} & \cdots & 0 \\
\vdots & \ddots & \vdots \\
0 & \cdots & B^{*} \omega_{N, k}
\end{array}\right) .
$$

Such a lower bound can only be made on some particular examples.

Note that, if $N=2, B$ is defined by (8.2) and

$$
M=\alpha\left(\begin{array}{ll}
0 & 1 \\
1 & 0
\end{array}\right)
$$

with $\alpha>0$, then we are back to the setting of Section 8.1. Indeed $M$ is symmetric with $A_{0}+M$ positive definite for $\alpha$ small enough, and diagonalized by the orthogonal matrix

$$
O=\frac{1}{\sqrt{2}}\left(\begin{array}{cc}
-1 & 1 \\
1 & 1
\end{array}\right)\left(\text { with } D=\alpha\left(\begin{array}{cc}
-1 & 0 \\
0 & 1
\end{array}\right)\right)
$$

We then finish this subsection by considering another example. Take $N=3$ and

$$
B\left(\begin{array}{l}
\omega_{1} \\
\omega_{2} \\
\omega_{3}
\end{array}\right)=\sqrt{\beta}\left(\begin{array}{l}
\omega_{1} \\
0 \\
0
\end{array}\right)+\sqrt{\gamma}\left(\begin{array}{l}
0 \\
\omega_{2} \\
0
\end{array}\right)+\sqrt{\delta}\left(\begin{array}{l}
0 \\
0 \\
\omega_{3}
\end{array}\right),
$$

with non negative real numbers $\beta, \gamma, \delta$, which is a bounded operator from $H$ into itself (i.e. $U=H$ ). We chose the matrix $M$ defined by

$$
M=\alpha\left(\begin{array}{lll}
0 & 1 & 0 \\
1 & 0 & 1 \\
0 & 1 & 0
\end{array}\right), \quad \alpha>0
$$

which is obviously symmetric. As previously we can verify that $A_{0}+M$ is positive definite if $\alpha<\pi^{2} / 2$. Moreover $M$ can be diagonalized by the orthogonal matrix

$$
O=\frac{1}{2}\left(\begin{array}{ccc}
1 & \sqrt{2} & 1 \\
-\sqrt{2} & 0 & \sqrt{2} \\
1 & -\sqrt{2} & 1
\end{array}\right)
$$

into

$$
D=\left(\begin{array}{ccc}
-\sqrt{2} \alpha & 0 & 0 \\
0 & 0 & 0 \\
0 & 0 & \sqrt{2} \alpha
\end{array}\right)
$$

Then the spectrum of $A=A_{0}+M$ is given by

$$
\operatorname{Sp}(A)=\left\{k^{2} \pi^{2}-\sqrt{2} \alpha\right\}_{k \in \mathbb{N}^{*}} \cup\left\{k^{2} \pi^{2}\right\}_{k \in \mathbb{N}^{*}} \cup\left\{k^{2} \pi^{2}+\sqrt{2} \alpha\right\}_{k \in \mathbb{N}^{*}},
$$

and the eigenvalues are simple (because the assumption $\alpha<\pi^{2} / 2$ implies that $k^{2} \pi^{2}+\sqrt{2} \alpha<(k+1)^{2} \pi^{2}-\sqrt{2} \alpha$ ). Moreover, as we have shown previously, the generalized gap condition (1.7) is satisfied with $M=3$. Thanks to $(8.8)$ the normalized eigenvectors are given by

$$
\omega_{1, k}=\frac{1}{\sqrt{2}}\left(\begin{array}{c}
1 \\
-\sqrt{2} \\
1
\end{array}\right) \sin (k \pi \cdot), \quad \omega_{2, k}=\frac{1}{\sqrt{2}}\left(\begin{array}{c}
\sqrt{2} \\
0 \\
-\sqrt{2}
\end{array}\right) \sin (k \pi \cdot), \quad \omega_{3, k}=\frac{1}{\sqrt{2}}\left(\begin{array}{c}
1 \\
\sqrt{2} \\
1
\end{array}\right) \sin (k \pi \cdot) .
$$


We set

$$
\alpha_{k}^{(1,2)}=\lambda_{2, k}-\lambda_{1, k}, \quad \alpha_{k}^{(1,3)}=\lambda_{3, k}-\lambda_{1, k}, \quad \alpha_{k}^{(2,3)}=\lambda_{3, k}-\lambda_{2, k} .
$$

Therefore, for all $C=\left(c_{1}, c_{2}, c_{3}\right)^{T} \in \mathbb{R}^{3}$, we have

$$
\begin{aligned}
\left\|B_{k}^{-1} \Phi_{k} C\right\|_{U, 2}^{2}= & \left\|\left(\begin{array}{ccc}
1 & 1 & 1 \\
0 & \alpha_{k}^{(1,2)} & \alpha_{k}^{(1,3)} \\
0 & 0 & \alpha_{k}^{(1,3)} \alpha_{k}^{(2,3)}
\end{array}\right)\left(\begin{array}{ccc}
B^{*} \omega_{1, k} & 0 & 0 \\
0 & B^{*} \omega_{2, k} & 0 \\
0 & 0 & B^{*} \omega_{3, k}
\end{array}\right)\left(\begin{array}{c}
c_{1} \\
c_{2} \\
c_{3}
\end{array}\right)\right\|_{U, 2}^{2} \\
= & \left\|c_{1} B^{*} \omega_{1, k}+c_{2} B^{*} \omega_{2, k}+c_{3} B^{*} \omega_{3, k}\right\|_{H}^{2}+\left\|c_{2} \alpha_{k}^{(1,2)} B^{*} \omega_{2, k}+c_{3} \alpha_{k}^{(1,3)} B^{*} \omega_{3, k}\right\|_{H}^{2} \\
& +\left|c_{3}\right|^{2}\left|\alpha_{k}^{(1,3)} \alpha_{k}^{(2,3)}\right|^{2}\left\|B^{*} \omega_{3, k}\right\|_{H}^{2} .
\end{aligned}
$$

After some calculations, we obtain

$$
\begin{aligned}
\left\|B_{k}^{-1} \Phi_{k} C\right\|_{U, 2}^{2}= & \frac{\beta}{4}\left(c_{1}+\sqrt{2} c_{2}+c_{3}\right)^{2}+\frac{\gamma}{2}\left(c_{3}-c_{1}\right)^{2}+\frac{\delta}{4}\left(c_{1}-\sqrt{2} c_{2}+c_{3}\right)^{2}+\frac{\beta}{4}\left(\sqrt{2} \alpha_{k}^{(1,2)} c_{2}+\alpha_{k}^{(1,3)} c_{3}\right)^{2} \\
& +\frac{\gamma}{2}\left|c_{3} \alpha_{k}^{(1,3)}\right|^{2}+\frac{\delta}{2}\left(-\sqrt{2} \alpha_{k}^{(1,2)} c_{2}+\alpha_{k}^{(1,3)} c_{3}\right)^{2}+\frac{\left|c_{3}\right|^{2}}{2}\left|\alpha_{k}^{(1,3)} \alpha_{k}^{(2,3)}\right|^{2}\left(\frac{\beta+\delta}{2}+\gamma\right) .
\end{aligned}
$$

Hence different decay results can be obtained for system (8.7) according to the values of $\beta, \gamma$ and $\delta$.

First if $\beta, \gamma, \delta>0$, then we have

$$
\left\|B_{k}^{-1} \Phi_{k} C\right\|_{U, 2}^{2} \geq C\left(c_{1}^{2}+c_{2}^{2}+c_{3}^{2}\right)
$$

for $C>0$, which shows that (1.10) holds and therefore system (8.7) is exponentially stable.

Second if $\gamma=0$ and $\beta, \delta>0$, we have

$$
\begin{aligned}
\left\|B_{k}^{-1} \Phi_{k} C\right\|_{U, 2}^{2} \geq & \frac{\min \{\beta, \delta\}}{4}\left(2 c_{1}^{2}+4 c_{2}^{2}+2 c_{3}^{2}+4 c_{1} c_{3}+\min \left\{\alpha_{k}^{(1,2)}, \alpha_{k}^{(1,3)}\right\}^{2}\left(4 c_{2}^{2}+2 c_{3}^{2}\right)\right. \\
& \left.+\min \left\{\alpha_{k}^{(1,3)}, \alpha_{k}^{(2,3)}\right\}^{4} c_{3}^{2}\right) \\
\geq & \frac{\min \{\beta, \delta\}}{4}\left(\left(2-\frac{2}{\epsilon}\right) c_{1}^{2}+4\left(1+\min \left\{\alpha_{k}^{(1,2)}, \alpha_{k}^{(1,3)}\right\}^{2}\right) c_{2}^{2}\right. \\
& \left.+\left(2-2 \epsilon+2 \min \left\{\alpha_{k}^{(1,2)}, \alpha_{k}^{(1,3)}\right\}^{2}\right) c_{3}^{2}\right),
\end{aligned}
$$

by Young's inequality with $\epsilon>0$. We then take $\epsilon=1+\min \left\{\alpha_{k}^{(1,2)}, \alpha_{k}^{(1,3)}\right\}^{2} / 2$, which implies

$$
2-\frac{2}{\epsilon}>\frac{\min \left\{\alpha_{k}^{(1,2)}, \alpha_{k}^{(1,3)}\right\}^{2}}{2}, \quad 2-2 \epsilon+2 \min \left\{\alpha_{k}^{(1,2)}, \alpha_{k}^{(1,3)}\right\}^{2}=\min \left\{\alpha_{k}^{(1,2)}, \alpha_{k}^{(1,3)}\right\}^{2},
$$

if $k$ is large enough. Consequently if $k$ is large enough, we have obtained that

$$
\begin{aligned}
\left\|B_{k}^{-1} \Phi_{k} C\right\|_{U, 2}^{2} & \geq \frac{\min \{\beta, \delta\}}{4}\left(\frac{\min \left\{\alpha_{k}^{(1,2)}, \alpha_{k}^{(1,3)}\right\}^{2}}{2} c_{1}^{2}+4 \min \left\{\alpha_{k}^{(1,2)}, \alpha_{k}^{(1,3)}\right\}^{2} c_{2}^{2}+\min \left\{\alpha_{k}^{(1,2)}, \alpha_{k}^{(1,3)}\right\}^{2} c_{3}^{2}\right) \\
& \geq \frac{\min \{\beta, \delta\}}{8} \min \left\{\alpha_{k}^{(1,2)}, \alpha_{k}^{(1,3)}\right\}^{2}\left(c_{1}^{2}+c_{2}^{2}+c_{3}^{2}\right),
\end{aligned}
$$

which shows that (1.13) holds with $l=1$, since $\min \left\{\alpha_{k}^{(1,2)}, \alpha_{k}^{(1,3)}\right\}^{2} \sim \lambda_{1, k}^{-2}$. 
We construct the space $V_{h}$ like in the previous subsection, i.e. it is the span of $\left(e_{i}, e_{j}, e_{k}\right)_{i, j, k \in\{1, \ldots, N\}}$, that still satisfies (1.5) and (1.6) with $\theta=1$.

Consequently, in the first case $(\beta, \gamma, \delta>0)$, we can apply Theorem 1.2 and thus the family of systems (1.9) is uniformly exponentially stable. In the second case $(\beta, \delta>0$ and $\gamma=0)$, we can apply Theorem 1.6 with $l=2$ and thus the family of systems (1.14) is uniformly polynomially stable, in the sense that (8.3) holds.

\section{REFERENCES}

[1] F. Alabau, P. Cannarsa and V. Komornik, Indirect internal stabilization of weakly coupled evolution equations. J. Evol. Equ. 2 (2002) $127-150$.

[2] H. Amann, Linear and Quasilinear Parabolic Problems: abstract linear theory,Springer-Verlag. Birkhäuser 1 (1995).

[3] K. Ammari and M. Tucsnak, Stabilization of second order evolution equations by a class of unbounded feedbacks. ESAIM: COCV 6 (2001) 361-386.

[4] I. Babuska and J. Osborn, Eigenvalue problems, in Handbook of Numerical Analysis II Finite Element Methods. Edited by P.G. Ciarlet and J.L. Lions. North-Holland, Amsterdam (1991).

[5] C. Baiocchi, V. Komornik and P. Loreti, Ingham-Beurling type theorems with weakened gap conditions. Acta Math. Hungar. 97 (2002) 55-95.

[6] H. T. Banks, K. Ito and C. Wang, Exponentially stable approximations of weakly damped wave equations, in Estimation and control of distributed parameter systems (Vorau, 1990), Internat. Ser. Numer. Math., vol. 100. Birkhäuser, Basel (1991) 1-33.

[7] A. Bátkai, K.-J. Engel, J. Prüss and R. Schnaubelt, Polynomial stability of operator semigroups. Math. Nachr. 279 (2006) $1425-1440$

[8] C.J.K. Batty and T. Duyckaerts, Non-uniform stability for bounded semi-groups on Banach spaces. J. Evol. Equ. 8 (2008) $765-780$.

[9] A. Borichev and Y. Tomilov, Optimal polynomial decay of functions and operator semigroups. Math. Ann. 347 (2010) 455-478.

[10] C. Castro and S. Micu, Boundary controllability of a linear semi-discrete 1-D wave equation derived from a mixed finite element method. Numer. Math. 102 (2006) 413-462.

[11] C. Castro, S. Micu and A. Münch, Numerical approximation of the boundary control for the wave equation with mixed finite elements in a square. IMA J. Numer. Anal. 28 (2008) 186-214.

[12] P. G. Ciarlet, The finite element method for elliptic problems. North-Holland, Amsterdam (1978).

[13] K. Engel and R. Nagel, One-parameter semigroups for linear evolution equations. Encyclopedia of Mathematics and its Applications. Springer-Verlag, New York (2000).

[14] S. Ervedoza, Spectral conditions for admissibility and observability of wave systems: applications to finite element schemes. Numer. Math. 113 (2009) 377-415.

[15] S. Ervedoza and E. Zuazua, Uniformly exponentially stable approximations for a class of damped systems. J. Math. Pures Appl. 91 (2009) 20-48.

[16] R. Glowinski, Ensuring well-posedness by analogy: Stokes problem and boundary control for the wave equation. J. Comput. Phys. 103 (1992) 189-221.

[17] R. Glowinski, W. Kinton and M.F. Wheeler, A mixed finite element formulation for the boundary controllability of the wave equation. Internat. J. Numer. Methods Engrg. 27 (1989) 623-635.

[18] R. Glowinski, C.H. Li and J.-L. Lions, A numerical approach to the exact boundary controllability of the wave equation. I. Dirichlet controls: description of the numerical methods. Japan J. Appl. Math. 7 (1990) 1-76.

[19] R. Glowinski and J.-L. Lions, Exact and approximate controllability for distributed parameter systems, in Acta numerica, Cambridge Univ. Press, Cambridge (1995) 159-333.

[20] E. Hewitt and K. Stromberg, Real and Abstract Analysis. Springer-Verlag, New York (1965).

[21] F.L. Huang, Characteristic conditions for exponential stability of linear dynamical systems in Hilbert spaces. Ann. Differ. Equ. 1 (1985) 43-56.

[22] J. A. Infante and E. Zuazua, Boundary observability for the space semi-discretizations of the one-dimensional wave equation. ESAIM: M2AN 33 (1999) 407-438.

[23] K. Ito and F. Kappel, The Trotter-Kato theorem and approximation of PDEs. Math. Comput. 67 (1998) $21-44$.

[24] Y. Latushkin and R. Shvydkoy, Hyperbolicity of semigroups and Fourier multipliers, in Systems, approximation, singular integral operators, and related topics (Bordeaux, 2000), Oper. Theory Adv. Appl. 129 (2001) 341-363.

[25] L. León and E. Zuazua, Boundary controllability of the finite-difference space semi-discretizations of the beam equation. ESAIM: COCV 8 (2002) 827-862. A tribute to J.L. Lions.

[26] Z. Liu and S. Zheng, Semigroups associated with dissipative systems, volume 398 of Chapman and Hall/CRC Research Notes in Mathematics. Chapman and Hall/CRC, Boca Raton, FL (1999).

[27] A. Münch, A uniformly controllable and implicit scheme for the 1-D wave equation. ESAIM: M2AN 39 (2005) $377-418$.

[28] M. Negreanu and E. Zuazua, A 2-grid algorithm for the 1-d wave equation, in Mathematical and numerical aspects of wave propagation-WAVES 2003. Springer, Berlin (2003) 213-217.

[29] S. Nicaise and J. Valein, Stabilization of second order evolution equations with unbounded feedback with delay. ESAIM: COCV 16 (2010) 420-456. 
[30] A. Pazy, Semigroups of linear operators and applications to partial differential equations, Applied Math. Sciences. SpringerVerlag, New York 44 (1983).

[31] K. Ramdani, T. Takahashi and M. Tucsnak, Uniformly exponentially stable approximations for a class of second order evolution equations - application to LQR problems. ESAIM: COCV 13 (2007) 503-527.

[32] P.-A. Raviart and J.-M. Thomas, Introduction l'analyse des équations aux dérivies partielles. Dunod, Paris (1998).

[33] D.-H. Shi and D.-X. Feng, Characteristic conditions of the generation of $C_{0}$ semigroups in a Hilbert space. J. Math. Anal. Appl. 247 (2000) 356-376.

[34] L.R. Tcheugoué Tébou and E. Zuazua, Uniform exponential long time decay for the space semi-discretization of a locally damped wave equation via an artificial numerical viscosity. Numer. Math. 95 (2003) 563-598.

[35] E. Zuazua, Boundary observability for the finite-difference space semi-discretizations of the 2-d wave equation in the square. J. Math. pures et appl. 78 (1999) 523-563. 